\title{
A Pauson-Khand and Ring-Expansion Approach to the Aquariane Ring System
}

\author{
Paul D. Thornton and D. Jean Burnell* \\ Department of Chemistry, Dalhousie University, Halifax, Nova Scotia, Canada B3H 4J3 \\ Table of Contents
}

Procedures and spectral data

S2 - S17

Compounds 9 and $\mathbf{8}$

Compounds 12 and 13 (two reduction methods)

Protection of $\mathbf{1 2}$

Vinyl addition and compound $\mathbf{1 4}$

Compounds 15 and 16

Compounds 17 and 18

Compound 19

Compound $\mathbf{2 0}$ and hydrolysis of $\mathbf{1 7}$

Compound 23

$\mathrm{S} 10$

Compounds 24 and 25

S11

Compounds 26 and 27

S12

Protection of $\mathbf{1 3}$

S13

TMS-7 and Compound 7

S14

Compounds 28 and 29

S15

Compound 30

S16

Compound 31

S17

${ }^{1} \mathrm{H}$ and ${ }^{13} \mathrm{C}$ NMR spectra

S18 - S43

ORTEP plots

S44 - S46

Compound 15

S44

Compound 17

S45

Compound 18

S46 


\section{General}

NMR spectra were acquired at $500 \mathrm{MHz}$ for ${ }^{1} \mathrm{H}$ and $125 \mathrm{MHz}$ for ${ }^{13} \mathrm{C}$, as $\mathrm{CDCl}_{3}$ solutions except where indicated. ${ }^{13} \mathrm{C}$ NMR shifts are followed in brackets by the number of attached hydrogens.

\section{1-(Trimethylsilyl)hept-6-en-1-yn-3-one (9)}

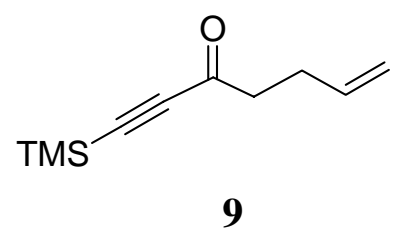

After the procedure of Yamaguchi et al., ${ }^{5} 25.5 \mathrm{~mL}$ of $n$-BuLi in hexanes (1.44 M, $\left.36.8 \mathrm{mmol}\right)$ was added to a solution of ethynyltrimethylsilane $(3.61 \mathrm{~g}, 36.8 \mathrm{mmol})$ in THF $(45 \mathrm{~mL})$ at $-78^{\circ} \mathrm{C}$. The solution was stirred at $-78{ }^{\circ} \mathrm{C}$ for $15 \mathrm{~min}$ before a solution of benzyl pent-4-enoate $(3.50 \mathrm{~g}, 18.4 \mathrm{mmol})$ and $\mathrm{BF}_{3} \cdot \mathrm{OEt}_{2}(4.62 \mathrm{~mL}, 36.8 \mathrm{mmol})$ in $\mathrm{THF}(15 \mathrm{~mL})$ was added dropwise over $10 \mathrm{~min}$. The mixture was stirred at $-78{ }^{\circ} \mathrm{C}$ for $3 \mathrm{~h}$ before it was warmed to $\mathrm{rt}$, and the reaction was quenched with saturated aqueous $\mathrm{NH}_{4} \mathrm{Cl}$. The layers were separated, and the aqueous layer was extracted with $\mathrm{CH}_{2} \mathrm{Cl}_{2}(4 \times 30$ $\mathrm{mL}$ ). The combined organic layers were washed with brine and dried over $\mathrm{MgSO}_{4}$. The solvent was removed under reduced pressure, and flash chromatography (4\% EtOAc/hexanes) of the residue yielded $9(2.87 \mathrm{~g}, 85 \%)$ as a yellow oil. IR (KBr disc): $3080(\mathrm{~m}), 2963(\mathrm{~s}), 2152(\mathrm{~m}), 1680(\mathrm{~s}) \mathrm{cm}^{-1} ;{ }^{1} \mathrm{H}$ NMR: $\delta$ $5.81(1 \mathrm{H}, \mathrm{ddt}, J=17.1,10.3,6.4 \mathrm{~Hz}), 5.06(1 \mathrm{H}, \mathrm{dq}, J=17.1,1.5 \mathrm{~Hz}), 5.01(1 \mathrm{H}, \mathrm{dd}, J=10.3,1.5 \mathrm{~Hz})$, $2.66(2 \mathrm{H}, \mathrm{t}, J=7.3 \mathrm{~Hz}), 2.41(2 \mathrm{H}, \mathrm{m}), 0.25(9 \mathrm{H}, \mathrm{s}) ;{ }^{13} \mathrm{C} \mathrm{NMR}: \delta 187.0(0), 136.4$ (1), 115.8 (2), 102.0 (0), 98.1 (0), 44.5 (2), 28.0 (2), -0.6 (3C, 3) ); HRMS: $m / z 180.0975\left(\mathrm{M}^{+}\right)$, required for $\mathrm{C}_{10} \mathrm{H}_{16} \mathrm{OSi}$ : 180.0970 .

\section{2-(But-3-enyl)-2-((trimethylsilyl)ethynyl)cyclopentane-1,3-dione (8)}

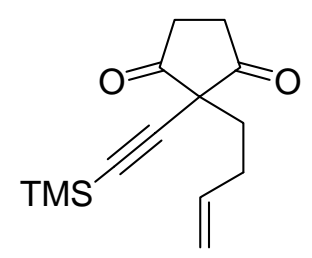

8

$\mathrm{BF}_{3} \cdot \mathrm{OEt}_{2}(2.60 \mathrm{~mL}, 20.7 \mathrm{mmol})$ was added to a solution of 9 (2.50 g, $\left.13.8 \mathrm{mmol}\right)$ in $\mathrm{CH}_{2} \mathrm{Cl}_{2}(60$ $\mathrm{mL})$ at $-78{ }^{\circ} \mathrm{C}$. The solution became yellow as was stirred at $-78{ }^{\circ} \mathrm{C}$ for $15 \mathrm{~min}$. Neat bis[(trimethylsilyl)oxy]cyclobutene (10) (4.73 g, $20.7 \mathrm{mmol})$ was added dropwise at $-78{ }^{\circ} \mathrm{C}$, and the mixture was stirred for $1 \mathrm{~h}$, after which time TLC analysis indicated that none of the compound 9 still 
remained. The mixture was warmed to $\mathrm{rt}$ and was treated sequentially with $\mathrm{H}_{2} \mathrm{O}(4.0 \mathrm{~mL}, 220 \mathrm{mmol})$ and $\mathrm{BF}_{3} \cdot \mathrm{OEt}_{2}(17.3 \mathrm{~mL}, 138 \mathrm{mmol})$. The mixture became red, and it was stirred for a further $1.5 \mathrm{~h}$. The mixture was filtered through a plug of silica gel $\left(\mathrm{CH}_{2} \mathrm{Cl}_{2}\right.$ as the eluent), and the solvent was removed in vacuo to yield $8(2.55 \mathrm{~g}, 74 \%)$ as colorless crystals, which were used without further purification. mp: 68-70 ${ }^{\circ} \mathrm{C}$; IR $\left(\mathrm{CH}_{2} \mathrm{Cl}_{2}\right): 3054$ (s), 2987 (s), 2306 (m), $1779(\mathrm{sh}), 1736(\mathrm{~s}) \mathrm{cm}^{-1} ;{ }^{1} \mathrm{H}$ NMR: $\delta 5.71(1 \mathrm{H}$, ddt, $J=17.0,10.3,6.7 \mathrm{~Hz}), 4.99(1 \mathrm{H}, \mathrm{dd}, J=17.0,1.6 \mathrm{~Hz}), 4.96(1 \mathrm{H}, \mathrm{dd}, J=10.2,1.6 \mathrm{~Hz}), 3.02(2 \mathrm{H}$, m), $2.73(2 \mathrm{H}, \mathrm{m}), 2.17$ (2H, m), 1.95 (2H, m), $0.14(9 \mathrm{H}, \mathrm{s}) ;{ }^{13} \mathrm{C}$ NMR: $\delta 207.6(2 \mathrm{C}, 0), 137.2(1), 115.9$ (2), 97.7 (0), 92.2 (0), 56.2 (0), 35.0 (2C, 2), 32.5 (2), 28.8 (2), -0.2 (3C, 3); HRMS: m/z 248.1227 $\left(\mathrm{M}^{+}\right)$, required for $\mathrm{C}_{14} \mathrm{H}_{20} \mathrm{O}_{2} \mathrm{Si}: 248.1233$.

$\left(2 R^{*}, 3 R^{*}\right)-(12)$ and $\left(2 R^{*}, 3 S^{*}\right)-2$-(But-3-enyl)-3-hydroxy-2-((trimethylsilyl)ethynyl)cyclopentanone (13)

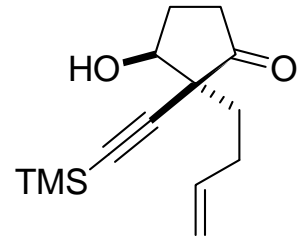

12

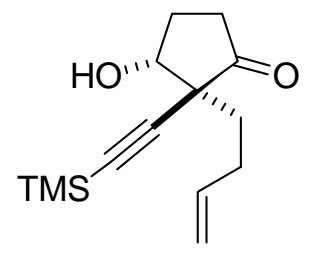

13

Reduction with lithium tri(tert-butoxy)aluminohydride. A $1 \mathrm{M}$ solution of lithium tri(tertbutoxy)aluminohydride $(11.9 \mathrm{~mL}, 11.9 \mathrm{mmol})$ was added dropwise over $10 \mathrm{~min}$ to a solution of diketone $8(2.70 \mathrm{~g}, 10.8 \mathrm{mmol})$ in THF $(108 \mathrm{~mL})$ at $-78{ }^{\circ} \mathrm{C}$. The solution was stirred at $-78{ }^{\circ} \mathrm{C}$ for $1 \mathrm{~h}$ before it was warmed to $0{ }^{\circ} \mathrm{C}$, and the reaction was quenched with excess saturated aqueous $\mathrm{NH}_{4} \mathrm{Cl}$. The mixture was acidified with $1 \mathrm{M} \mathrm{HCl}(25 \mathrm{~mL})$ and extracted with $\mathrm{CH}_{2} \mathrm{Cl}_{2}(4 \times 30 \mathrm{~mL})$. The combined organic layers were washed with brine and dried over $\mathrm{Na}_{2} \mathrm{SO}_{4}$. Evaporation of solvent under reduced pressure, followed by flash chromatography of the residue $\left(75 \% \mathrm{CH}_{2} \mathrm{Cl}_{2} /\right.$ hexanes $)$ gave $12(2.09 \mathrm{~g}, 78$ $\%)$ as a colorless oil and $13(0.510 \mathrm{~g}, 18 \%)$ as a white solid.

Reduction with triethylsilane. TFA $(5.0 \mathrm{~mL})$ was added to diketone $\mathbf{8}(250 \mathrm{mg}, 1.00 \mathrm{mmol})$ and the resulting yellow solution was cooled to $-10^{\circ} \mathrm{C}$. Triethylsilane $(0.175 \mathrm{~mL}, 1.10 \mathrm{mmol})$ was added, and the solution was stirred at $-10^{\circ} \mathrm{C}$ for $8 \mathrm{~h}$. The solution was poured into cold saturated aqueous $\mathrm{NaHCO}_{3}$ solution $(100 \mathrm{~mL})$, and the aqueous layer was extracted with $\mathrm{CH}_{2} \mathrm{Cl}_{2}(4 \times 25 \mathrm{~mL})$. The organic layers were combined, washed with brine, and dried over $\mathrm{Na}_{2} \mathrm{SO}_{4}$. Evaporation of the solvent under reduced pressure and flash chromatography of the residue $\left(\mathrm{CH}_{2} \mathrm{Cl}_{2}\right)$ gave $13(176 \mathrm{mg}, 70 \%)$ as a white solid in addition to a diol resulting from over-reduction (32 $\mathrm{mg}, 13 \%)$, and recovered $\mathbf{8}$ (25 $\mathrm{mg}, 10 \%)$. 
For 12: IR (film): 3543 (br), 3078 (m), 2959 (s), 2164 (s), 1748 (s) cm ${ }^{-1} ;{ }^{1} \mathrm{H}$ NMR: $\delta 5.81(1 \mathrm{H}$, $\mathrm{ddt}, J=17.1,10.2,6.6 \mathrm{~Hz}), 5.04(1 \mathrm{H}, \mathrm{dq}, J=17.1,1.7 \mathrm{~Hz}), 4.97(1 \mathrm{H}, \mathrm{dd}, J=10.2,1.7 \mathrm{~Hz}), 4.06(1 \mathrm{H}, \mathrm{q}$, $J=5.1 \mathrm{~Hz}), 2.59(1 \mathrm{H}, \mathrm{ddd}, J=19.1,9.6,7.0 \mathrm{~Hz}), 2.36-2.12(5 \mathrm{H}, \mathrm{m}), 2.05(1 \mathrm{H}, \mathrm{m}), 1.73(1 \mathrm{H}, \mathrm{ddd}, J=$ 13.7, 11.3, $5.5 \mathrm{~Hz}), 1.59(1 \mathrm{H}, \mathrm{ddd}, J=13.7,11.3,5.5 \mathrm{~Hz}), 0.19(9 \mathrm{H}, \mathrm{s}) ;{ }^{13} \mathrm{C}$ NMR: $\delta 212.0(0), 137.8$ (1), 115.3 (2), 100.4 (0), 93.8 (0), 75.7 (1), 57.4 (0), 34.5 (2), 33.1 (2), 29.2 (2), 27.7 (2), 0.2 (3C, 3); HRMS: $m / z$ 250.1384 $\left(\mathrm{M}^{+}\right)$, required for $\mathrm{C}_{14} \mathrm{H}_{22} \mathrm{O}_{2} \mathrm{Si}: 250.1389$.

For 13: mp: $46-47{ }^{\circ} \mathrm{C}$; IR $\left(\mathrm{CH}_{2} \mathrm{Cl}_{2}\right)$ : 3604 (br), 3054 (s), 2987 (s), 2306 (m), 1750 (s); ${ }^{1} \mathrm{H}$ NMR: $\delta 5.91(1 \mathrm{H}$, ddt $J=17.2,10.2,6.3 \mathrm{~Hz}), 5.11(1 \mathrm{H}, \mathrm{dq}, J=17.2,1.6 \mathrm{~Hz}), 5.01(1 \mathrm{H}, \mathrm{dd}, J=10.2,1.6 \mathrm{~Hz})$, 4.42 (1H, br s), 2.54-2.35 (4 H, m), $2.21(1 \mathrm{H}, \mathrm{m}), 1.97(1 \mathrm{H}, \mathrm{m}), 1.88(1 \mathrm{H}, \mathrm{ddd}, J=14.0,10.4,5.0 \mathrm{~Hz})$, $1.71(1 \mathrm{H}, \mathrm{s}) 1.64(1 \mathrm{H}, \mathrm{ddd}, J=14.0,10.4,6.3 \mathrm{~Hz}), 0.14(9 \mathrm{H}, \mathrm{s}) ;{ }^{13} \mathrm{C}$ NMR: $\delta 212.6(0), 138.6(1), 115.5$ (2), 102.6 (0), 91.4 (0), 76.2 (1), 55.5 (0), 33.3 (2), 29.3 (2), 28.4 (2), 28.3 (2), 0.1 (3C, 3); HRMS: m/z $250.1390\left(\mathrm{M}^{+}\right)$, required for $\mathrm{C}_{14} \mathrm{H}_{22} \mathrm{O}_{2} \mathrm{Si}: 250.1389$.

$\left(2 R^{*}, 3 R^{*}\right)-2-($ But-3-enyl)-3-(tert-butyldimethylsilyl)oxy-2-((trimethylsilyl)ethynyl)cyclopentanone

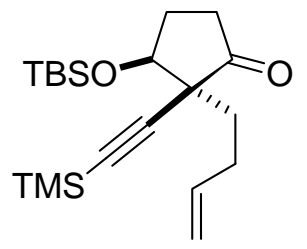

Triethylamine $(1.27 \mathrm{~mL}, 0.921 \mathrm{~g}, 9.10 \mathrm{mmol})$ was added to a solution of alcohol $12(2.09 \mathrm{~g}, 8.27$ $\mathrm{mmol})$ in THF $(60 \mathrm{~mL})$ and cooled to $0{ }^{\circ} \mathrm{C}$. TBSOTf $(1.62 \mathrm{~mL}, 9.1 \mathrm{mmol})$ was added dropwise. The solution was stirred for $30 \mathrm{~min}$ at $0{ }^{\circ} \mathrm{C}$, after which TLC analysis indicated the complete consumption of the starting material. The reaction was quenched with excess saturated aqueous $\mathrm{NaHCO}_{3}$, and the layers were separated. The aqueous layer was extracted with $\mathrm{CH}_{2} \mathrm{Cl}_{2}(4 \times 25 \mathrm{~mL})$. The combined organic layers were washed with brine and dried over $\mathrm{MgSO}_{4}$. Removal of the solvent under reduced pressure, and purification of the residue using flash chromatography (5\% EtOAc/hexanes) afforded the title compound (2.78 g, 92\%) as a colorless oil. IR $\left(\mathrm{CH}_{2} \mathrm{Cl}_{2}\right): 3054(\mathrm{~s}), 2957(\mathrm{~s}), 2306(\mathrm{~m}), 1747(\mathrm{~s}) \mathrm{cm}^{-1} ;{ }^{1} \mathrm{H}$ NMR: $\delta 5.81(1 \mathrm{H}, \mathrm{ddt}, J=17.1,10.3,6.6 \mathrm{~Hz}), 5.02(\mathrm{H}, \mathrm{dq}, J=17.1,1.5 \mathrm{~Hz}), 4.96(1 \mathrm{H}, \mathrm{dd}, J=10.3,1.5$ Hz), $4.10(1 \mathrm{H}, \mathrm{t}, J=7.8 \mathrm{~Hz}), 2.57(1 \mathrm{H}, \mathrm{ddd}, J=19.1,9.5,7.3 \mathrm{~Hz}), 2.35-2.15(3 \mathrm{H}, \mathrm{m}), 2.09(1 \mathrm{H}, \mathrm{m})$, $2.02(1 \mathrm{H}, \mathrm{m}), 1.65(1 \mathrm{H}, \mathrm{ddd}, J=13.4,11.8,5.2 \mathrm{~Hz}), 1.54(1 \mathrm{H}, \mathrm{ddd}, J=13.4,11.8,5.2 \mathrm{~Hz}), 0.94$ (9H, s), 0.14 (9H, s), 0.11 (6H, s); ${ }^{13} \mathrm{C}$ NMR: $\delta 212.7$ (0), 138.3 (1), 115.1 (2), 102.5 (0), 90.9 (0), 76.9 (1), 57.2 (0), 34.2 (2), 33.6 (2), 29.6 (2), 29.0 (2), 26.0 (3C, 3), 18.3 (0), 0.3 (3C, 3), -4.2 (3), -4.5 (3); HRMS: $m / z$ 364.2260 $\left(\mathrm{M}^{+}\right)$, required for $\mathrm{C}_{20} \mathrm{H}_{36} \mathrm{O}_{2} \mathrm{Si}_{2}: 364.2254$. 
$\left(1 R^{*}, 2 R^{*}, 3 R^{*}\right)$-2-(But-3-enyl)-3-(tert-butyldimethylsilyl)oxy-2-((trimethylsilyl)ethynyl)-1-vinylcyclopentan-1-ol

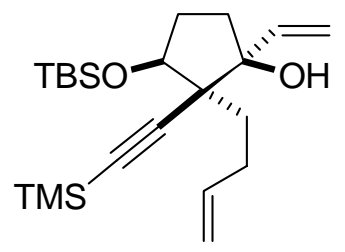

A $1 \mathrm{M}$ solution of vinylmagnesium bromide in THF $(12 \mathrm{~mL}, 12 \mathrm{mmol})$ was added to a suspension of anhydrous $\mathrm{CeCl}_{3}(2.95 \mathrm{~g}, 12.0 \mathrm{mmol})$ stirring in THF $(8 \mathrm{~mL})$ at $-78{ }^{\circ} \mathrm{C}$. The resulting suspension was stirred at this temperature for $2 \mathrm{~h}$. To this mixture was added a solution of $\left(2 R^{*}, 3 R^{*}\right)-2$ (but-3-enyl)-3-(tert-butyldimethylsilyl)oxy-2-((trimethylsilyl)ethynyl)cyclopentanone $\quad\left(\begin{array}{lll}1.75 & \mathrm{~g}, & 4.80\end{array}\right.$ mmol) in THF $(4.0 \mathrm{~mL})$ at $-78{ }^{\circ} \mathrm{C}$, and the mixture was stirred for $1 \mathrm{~h}$. After this time, TLC analysis indicated that no starting material remained. The solution was warmed to $\mathrm{rt}$, and the reaction was quenched with $6 \mathrm{~mL}$ of $5 \%$ aqueous $\mathrm{AcOH}$. The layers were separated, and the aqueous layer was extracted with $\mathrm{Et}_{2} \mathrm{O}(4 \times 20 \mathrm{~mL})$. The combined organic layers were washed with saturated aqueous $\mathrm{NaHCO}_{3}$ and brine, and then dried over $\mathrm{Na}_{2} \mathrm{SO}_{4}$. The solvent was removed in vacuo, and the residue was purified by flash chromatography (3\% EtOAc/hexanes) to give the title compound (1.73 g, 92\%) as a colorless oil. IR $\left(\mathrm{CH}_{2} \mathrm{Cl}_{2}\right)$ : 3468 (br), 3054 (s), 2957 (s), $2162(\mathrm{~m}), 1640(\mathrm{w}) \mathrm{cm}^{-1} ;{ }^{1} \mathrm{H}$ NMR: $\delta 5.88$ $(1 \mathrm{H}, \mathrm{ddd}, J=17.2,10.8,0.6 \mathrm{~Hz}), 5.80(1 \mathrm{H}, \mathrm{ddt}, J=17.0,10.3,6.7 \mathrm{~Hz}), 5.38(1 \mathrm{H}, \mathrm{dd}, J=17.3,1.7 \mathrm{~Hz})$, $5.20(1 \mathrm{H}, \mathrm{dd}, J=10.8,1.7 \mathrm{~Hz}), 5.01(1 \mathrm{H}, \mathrm{ddd}, J=17.2,10.3,1.7 \mathrm{~Hz}), 4.95(1 \mathrm{H}, \mathrm{dd}, J=10.3 \mathrm{~Hz}, 1.7$ $\mathrm{Hz}), 4.1(1 \mathrm{H}, \mathrm{dd}, J=5.7,2.6 \mathrm{~Hz}), 4.01(1 \mathrm{H}, \mathrm{d}, J=0.6 \mathrm{~Hz}), 2.37(1 \mathrm{H}, \mathrm{m}), 2.06-1.94(4 \mathrm{H}, \mathrm{m}), 1.89(1 \mathrm{H}$, m), $1.50(1 \mathrm{H}, \mathrm{ddd}, J=13.4,12.4,5.0 \mathrm{~Hz}), 1.15(1 \mathrm{H}, \mathrm{ddd}, J=13.4,12.4,5.0 \mathrm{~Hz}), 0.92(9 \mathrm{H}, \mathrm{s}), 0.15(9 \mathrm{H}$, s), $0.11(3 \mathrm{H}, \mathrm{s}), 0.10(3 \mathrm{H}, \mathrm{s}) ;{ }^{13} \mathrm{C}$ NMR: $\delta 138.9$ (1), 138.7 (1), 115.6 (2), 114.7 (2), $105.6(0), 90.9$ (0), 84.3 (0), 79.2 (1), 58.0 (0), 35.7 (2), 34.1 (2), 31.8 (2), 30.0 (2), 26.0 (3C, 3), 18.3 (0), 0.4 (3C, 3), -4.5 (3), -4.6 (3); HRMS: $m / z 392.2575\left(\mathrm{M}^{+}\right)$, required for $\mathrm{C}_{22} \mathrm{H}_{40} \mathrm{O}_{2} \mathrm{Si}_{2}: 392.2567$.

$\left(1 R^{*}, 2 R^{*}, 3 R^{*}\right)-2-($ But-3-enyl)-3-(tert-butyldimethylsilyl)oxy-2-ethynyl-1-vinylcyclopentan-1-ol (14)

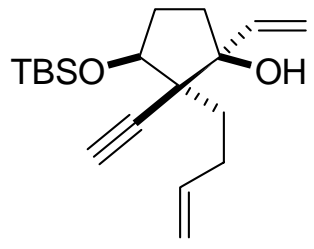

14

Anhydrous $\mathrm{K}_{2} \mathrm{CO}_{3}(0.790 \mathrm{~g}, 5.45 \mathrm{mmol})$ was added to a solution of $\left(1 \mathrm{R}^{*}, 2 \mathrm{R} *, 3 \mathrm{R} *\right)-2-($ but-3enyl)-3-(tert-butyldimethylsilyl)oxy-2-((trimethylsilyl)ethynyl)-1-vinyl-cyclopentan-1-ol (1.95 g, 4.96 
mmol) in methanol $(16 \mathrm{~mL})$ at $0{ }^{\circ} \mathrm{C}$. The mixture was warmed to $\mathrm{rt}$ and stirred for $6 \mathrm{~h}$. After this time the mixture was diluted with $\mathrm{H}_{2} \mathrm{O}$ and $\mathrm{Et}_{2} \mathrm{O}$, and the layers were separated. The aqueous layer was extracted with $\mathrm{Et}_{2} \mathrm{O}(5 \times 10 \mathrm{~mL})$. The combined organic layers were washed with brine and then dried over $\mathrm{Na}_{2} \mathrm{SO}_{4}$. The solvent was evaporated under reduced pressure, and the residue was purified using flash chromatography (5\% EtOAc / hexanes) to give $14(1.48 \mathrm{~g}, 93 \%)$ as a colorless oil. IR $\left(\mathrm{CH}_{2} \mathrm{Cl}_{2}\right)$ : 3465 (br), 3302 (m), 3054 (s), 2955 (s), 2306 (m), 1641 (w) $\mathrm{cm}^{-1} ;{ }^{1} \mathrm{H}$ NMR: $\delta 5.91$ (1H, dd, $J=17.3$, $10.8 \mathrm{~Hz}), 5.79(1 \mathrm{H}, \mathrm{ddt}, J=17.0,10.5,6.6 \mathrm{~Hz}), 5.43(1 \mathrm{H}, \mathrm{dd}, J=17.3,1.7 \mathrm{~Hz}), 5.23(1 \mathrm{H}, \mathrm{dd}, J=10.8$, $1.7 \mathrm{~Hz}), 5.03(1 \mathrm{H}, \mathrm{dq}, J=17.1,1.7 \mathrm{~Hz}), 4.96(1 \mathrm{H}, \mathrm{dd}, J=10.5,1.7 \mathrm{~Hz}), 4.34(1 \mathrm{H}$, br s), $4.18(1 \mathrm{H}, \mathrm{m})$, $2.40(1 \mathrm{H}, \mathrm{s}), 2.27(1 \mathrm{H}, \mathrm{s}), 2.17-2.02(4 \mathrm{H}, \mathrm{m}), 1.92(1 \mathrm{H}, \mathrm{m}) 1.55(1 \mathrm{H}, \mathrm{ddd}, J=13.7,12.2,4.8 \mathrm{~Hz}), 1.14$ $(1 \mathrm{H}, \mathrm{ddd}, J=13.7,12.2,4.8 \mathrm{~Hz}), 0.92(9 \mathrm{H}, \mathrm{s}), 0.13(3 \mathrm{H}, \mathrm{s}), 0.11(3 \mathrm{H}, \mathrm{s}) ;{ }^{13} \mathrm{C} \mathrm{NMR}: \delta 138.5(1), 138.3$ (1), 115.9 (2), 115.0 (2), 84.8 (0), 83.4 (1), 79.4 (1), 74.3 (0), 57.1 (0), 35.7 (2), 33.7 (2), 31.7 (2), 29.9 (2), 26.0 (3C, 3), $18.3(0),-4.5$ (3), -4.6 (3); HRMS: $m / z 320.2166\left(\mathrm{M}^{+}\right)$, required for $\mathrm{C}_{19} \mathrm{H}_{32} \mathrm{O}_{2} \mathrm{Si}$ : 320.2172 .

$\left(1 R^{*}, 2 R^{*}, 3 a^{\prime} R^{*}, 5 R^{*}\right)-\quad(15)$ and $\left(1 R^{*}, 2 R^{*}, 3 a^{\prime} S^{*}, 5 R^{*}\right)-5$-(tert-Butyldimethylsilyl)oxy-2',3',3a',4'tetrahydro-2-hydroxy-2-vinyl-5' $H$-spiro[cyclopentane-1,1'-pentalen]-5' -one (16)

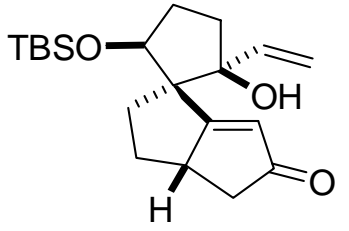

15

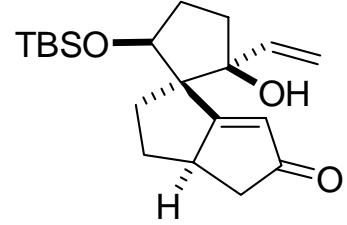

16

Dicobalt octacarbonyl (1.54 g, $4.50 \mathrm{mmol})$ was added to a solution of $\mathbf{1 4}(1.20 \mathrm{~g}, 3.74 \mathrm{mmol})$ in $\mathrm{CH}_{2} \mathrm{Cl}_{2}(15 \mathrm{~mL})$. The resulting red solution was stirred at $\mathrm{rt}$ for $8 \mathrm{~h}$, after which time TLC analysis indicated that no alcohol remained. The mixture was diluted with $\mathrm{CH}_{2} \mathrm{Cl}_{2}(220 \mathrm{~mL})$ and warmed to 30 ${ }^{\circ} \mathrm{C}$. A solution of anhydrous TMANO ${ }^{\dagger}(2.25 \mathrm{~g}, 29.9 \mathrm{mmol})$ in $\mathrm{CH}_{2} \mathrm{Cl}_{2}(160 \mathrm{~mL})$ was added. The mixture was stirred for $14 \mathrm{~h}$ at $30{ }^{\circ} \mathrm{C}$, during which time a deep blue precipitate formed. The mixture was filtered through a plug of silica gel (EtOAc as eluent) and concentrated in vacuo to afford a yellow oil. Flash chromatography (20\% EtOAc/hexanes) yielded $15(0.783$ g, 60\%) and 16 (0.072 g, 6\%) as colorless crystalline solids, and some starting material $14(0.118 \mathrm{~g}, 10 \%)$ was recovered.

For 15: mp: 78-80 ${ }^{\circ} \mathrm{C}$; IR $\left(\mathrm{CH}_{2} \mathrm{Cl}_{2}\right)$ : 3461(br), 3055 (m), 2957 (s), 1701 (s), $1621(\mathrm{~m}) \mathrm{cm}^{-1}$; ${ }^{1} \mathrm{H}$ NMR: $\delta 6.38(1 \mathrm{H}, \mathrm{d}, J=2.3 \mathrm{~Hz}), 5.77(1 \mathrm{H}, \mathrm{ddd}, J=17.1,10.8,1.0 \mathrm{~Hz}), 5.36(1 \mathrm{H}, \mathrm{dd}, J=17.3,1.3 \mathrm{~Hz})$,

\footnotetext{
$\dagger$ Anhydrous TMANO was obtained from TMANO dihydrate by azeotropic distillation in benzene.
} 
$5.14(1 \mathrm{H}, \mathrm{dd}, J=10.8,1.7 \mathrm{~Hz}), 4.83(1 \mathrm{H}, \mathrm{d}, J=1.0 \mathrm{~Hz}), 4.14(1 \mathrm{H}, \mathrm{d}, J=4.7 \mathrm{~Hz}), 2.92(1 \mathrm{H}, \mathrm{m}), 2.57$ $(1 \mathrm{H}, \mathrm{dd}, J=17.8,6.4 \mathrm{~Hz}), 2.24-2.01(5 \mathrm{H}, \mathrm{m}), 1.96(1 \mathrm{H}, \mathrm{dd}, J=17.8,5.0 \mathrm{~Hz}), 1.86(1 \mathrm{H}, \mathrm{dd}, J=13.5$, $7.9 \mathrm{~Hz}), 1.53(1 \mathrm{H}, \mathrm{ddd}, J=13.5,11.8,6.9 \mathrm{~Hz}), 1.13(1 \mathrm{H}, \mathrm{ddt}, J=10.1,8.1,11.9 \mathrm{~Hz}), 0.87(9 \mathrm{H}, \mathrm{s}), 0.05$ $(3 \mathrm{H}, \mathrm{s}),-0.02(3 \mathrm{H}, \mathrm{s}) ;{ }^{13} \mathrm{C}$ NMR: $\delta 211.1(0), 187.3$ (0), $137.6(1), 129.1(1), 116.5(2), 85.8(0), 83.9$ (1), 61.2 (0), 46.5 (1), 41.5 (2), 37.4 (2), 35.9 (2), 31.7 (2), 30.0 (2), 26.7 (3C, 3), 17.9 (0), -4.9 (3), -5.0 (3); HRMS: $m / z 348.2114\left(\mathrm{M}^{+}\right)$, required for $\mathrm{C}_{20} \mathrm{H}_{32} \mathrm{O}_{3} \mathrm{Si}$ : 348.2121.

For 16: mp: 66-68 ${ }^{\circ} \mathrm{C}$; IR $\left(\mathrm{CH}_{2} \mathrm{Cl}_{2}\right)$ : 3469 (br), 3055 (m), 2956 (s), 1700 (s), 1620 (m) cm ${ }^{-1} ;{ }^{1} \mathrm{H}$ NMR: $\delta 6.16(1 \mathrm{H}, \mathrm{d}, J=2.3 \mathrm{~Hz}), 5.87(1 \mathrm{H}, \mathrm{ddd}, J=17.2,10.8,0.8 \mathrm{~Hz}), 5.37(1 \mathrm{H}, \mathrm{dd}, J=17.2,1.7 \mathrm{~Hz})$, $5.22(1 \mathrm{H}, \mathrm{dd}, J=10.8,1.7 \mathrm{~Hz}), 4.40(1 \mathrm{H}, \mathrm{s}), 4.02(1 \mathrm{H}, \mathrm{dd}, J=6.0,1.2 \mathrm{~Hz}), 2.88(1 \mathrm{H}, \mathrm{m}), 2.58(1 \mathrm{H}, \mathrm{dd}$, $J=17.5,6.3 \mathrm{~Hz}), 2.29(1 \mathrm{H}, \mathrm{m}), 2.13-1.92(6 \mathrm{H}, \mathrm{m}), 1.46(1 \mathrm{H}, \mathrm{dd}, J=13.3,7.3 \mathrm{~Hz}), 1.16(1 \mathrm{H}, \mathrm{ddt}, J=$ 19.4, 7.5, $11.9 \mathrm{~Hz}), 0.92(9 \mathrm{H}, \mathrm{s}), 0.07$ (3H, s), $0.02(3 \mathrm{H}, \mathrm{s}) ;{ }^{13} \mathrm{C}$ NMR: $\delta 210.6$ (0), $187.6(0), 138.3$ (1), 129.1 (1), 116.5 (2), 85.3 (0), 82.3 (1), 62.1 (0), 48.7 (1), 42.2 (2), 36.2 (2), 35.5 (2), 33.2 (2), 29.8 (2), 25.8 (3C, 3), $17.8(0),-4.6$ (3), -4.9 (3); HRMS: $m / z 348.2117\left(\mathrm{M}^{+}\right)$, required for $\mathrm{C}_{20} \mathrm{H}_{32} \mathrm{O}_{3} \mathrm{Si}$ : 348.2121 .

$\left(4 a R^{*}, 7 R^{*}, 7 a R^{*}, 9 a R^{*}\right)-7-(t e r t-B u t y l d i m e t h y l s i l y l) o x y-3,4,4 a, 5,6,7,9,9 a-o c t a h y d r o-4 a-h y d r o x y-1 H-$ cyclopent $[k l]$-as-indacen-2(8H)-one $(17)$ and $\left(2 a R^{*}, 4 a S^{*}, 5 R^{*}, 6 a R^{*}, 9 a S^{*}, 9 b R^{*}\right)-5$-(tert-butyldimethylsilyl)oxy-2,2a,3,4,4a,5,6,6a,9,9a-decahydro-1H-pentaleno[1,6-dc]indene-1,7(8H)-dione (18)

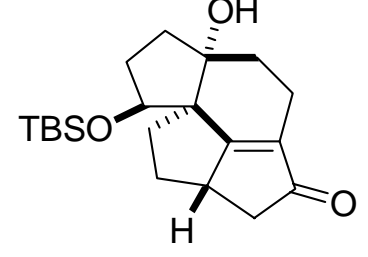

17

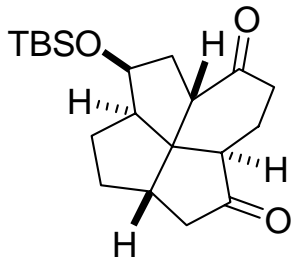

18

Potassium hydroxide $(0.053 \mathrm{~g}, 9.5 \mathrm{mmol})$ was added in one portion to a solution of $15(0.300 \mathrm{~g}$, $8.60 \mathrm{mmol})$ in $\mathrm{MeOH}(15 \mathrm{~mL})$ at $0{ }^{\circ} \mathrm{C}$. The reaction was allowed to warm to $\mathrm{rt}$ and stirred for $16 \mathrm{~h}$. The reaction was quenched with saturated aqueous $\mathrm{NH}_{4} \mathrm{Cl}$ and $\mathrm{MeOH}$ was removed in vacuo. The resulting mixture was extracted with $\mathrm{CH}_{2} \mathrm{Cl}_{2}(4 \times 10 \mathrm{~mL})$. The organic layers were combined, washed with brine, and then dried over $\mathrm{Na}_{2} \mathrm{SO}_{4}$. Evaporation of the solvent and flash chromatography (35\% EtOAc/hexanes) provided $17(0.25 \mathrm{~g}, 83 \%)$ and $18(0.018,6 \%)$ as colorless crystalline solids.

For 17: mp: $114-116{ }^{\circ} \mathrm{C}$; IR (KBr disc): 3445 (br), 2953 (s), 1692 (s), 1662 (s) cm ${ }^{-1} ;{ }^{1} \mathrm{H}$ NMR: $\delta$ $4.15(1 \mathrm{H}, \mathrm{dd}, J=5.7,3.4 \mathrm{~Hz}), 3.00(1 \mathrm{H}, \mathrm{m}), 2.74(1 \mathrm{H}, \mathrm{dd}, J=18.5,6.1 \mathrm{~Hz}), 2.35(1 \mathrm{H}, \mathrm{dt}, J=13.6,7.8$ Hz), 2.30-2.03 (7H, m), 1.83 (1H, ddd, $J=13.4,10.2,6.3 \mathrm{~Hz}), 1.71(2 \mathrm{H}, \mathrm{m}), 1.54(1 \mathrm{H}, \mathrm{m}), 1.24(1 \mathrm{H}, \mathrm{br}$ 
s), $1.01(1 \mathrm{H}, \mathrm{m}) ;{ }^{13} \mathrm{C}$ NMR: $\delta 208.9(0), 185.2(0), 135.8(0), 81.3$ (1), $79.2(0), 58.5(0), 43.5$ (2), 40.5 (1), 37.0 (2), 34.5 (2), 33.4 (2), 32.0 (2), 30.7 (2), 25.6 (3C, 3), 17.8 (0), 17.3 (2), -4.6 (3), -5.1 (3); HRMS: $m / z, 348.2113\left(\mathrm{M}^{+}\right)$, required for $\mathrm{C}_{20} \mathrm{H}_{32} \mathrm{O}_{3} \mathrm{Si}: 348.2121$.

For 18: mp: $102-103{ }^{\circ} \mathrm{C}$; IR $\left(\mathrm{CH}_{2} \mathrm{Cl}_{2}\right): 2986$ (s), 1737 (s), 1709 (s) cm ${ }^{-1} ;{ }^{1} \mathrm{H}$ NMR $\left(\mathrm{C}_{6} \mathrm{D}_{6}\right): \delta$ $4.07(1 \mathrm{H}, \mathrm{ddd}, J=9.0,7.8,6.1 \mathrm{~Hz}), 2.51(1 \mathrm{H}, \mathrm{ddd}, J=12.2,5.8,2.6 \mathrm{~Hz}), 2.12(1 \mathrm{H}, \mathrm{dq}, J=13.6,4.9$ Hz), 2.03 (2H, m), 1.91-1.82 (4H, m), 1.72-1.58 (4H, m), $1.47(2 \mathrm{H}, \mathrm{m}), 1.31(1 \mathrm{H}, \mathrm{m}), 0.98(9 \mathrm{H}, \mathrm{s})$, $0.93(1 \mathrm{H}, \mathrm{m}), 0.09(3 \mathrm{H}, \mathrm{s}), 0.07(3 \mathrm{H}, \mathrm{s}) ;{ }^{13} \mathrm{C} \mathrm{NMR}\left(\mathrm{C}_{6} \mathrm{D}_{6}\right): \delta 216.6(0), 209.5(0), 73.5(1), 64.1(0), 54.2$ (1), 52.4 (1), 52.0 (1), 47.8 (1), 41.0 (2), 37.8 (2), 35.1 (2), 34.1 (2), 27.2 (2), 26.4 (3C, 3), 23.0 (2), 18.7 (0), -4.2 (3), -4.6 (3); HRMS: $m / z 348.2123\left(\mathrm{M}^{+}\right)$, required for $\mathrm{C}_{20} \mathrm{H}_{32} \mathrm{O}_{3} \mathrm{Si}: 348.2121$.

$\left(1 R^{*}, 2 R^{*}, 3 a^{\prime} R^{*}, 5 R^{*}, 5 \mathrm{a}^{\prime} S^{*}\right)-5$-(tert-Butyldimethylsilyl)oxy-2',3',3a',4'-tetrahydro-2-vinyl-5' $H$ spiro[cyclopentane-1,1'-pentalene]-2,5'-diol (19)

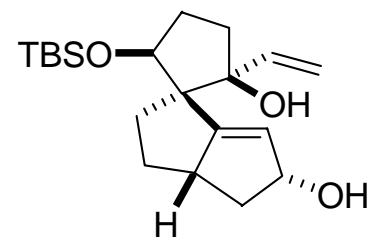

19

$\mathrm{CeCl}_{3} \cdot 7 \mathrm{H}_{2} \mathrm{O}$ (32 $\left.\mathrm{mg}, 0.086 \mathrm{mmol}\right)$ was added to a solution of 15 (20 mg, $\left.0.057 \mathrm{mmol}\right)$ in methanol $(1.0 \mathrm{~mL})$ and cooled to $0{ }^{\circ} \mathrm{C} . \mathrm{NaBH}_{4}(4 \mathrm{mg}, 0.1 \mathrm{mmol})$ was added in one portion, and the mixture was stirred at $0{ }^{\circ} \mathrm{C}$ for $1 \mathrm{~h}$, after which time TLC analysis indicated that no starting material remained. The reaction was quenched with saturated aqueous $\mathrm{NH}_{4} \mathrm{Cl}(1 \mathrm{~mL})$, and the resulting mixture was extracted with $\mathrm{CH}_{2} \mathrm{Cl}_{2}(5 \times 4 \mathrm{~mL})$. The combined organic layers were washed with brine, and then dried over $\mathrm{Na}_{2} \mathrm{SO}_{4}$. Removal of the solvent in vacuo yielded 19 (19 mg, 95\%) as a colorless oil that was used without further purification. IR (KBr disc): 3476 (br), $1655 \mathrm{~cm}^{-1} ;{ }^{1} \mathrm{H}$ NMR: $\delta 5.89(1 \mathrm{H}$, ddd, $J=$ 17.0, 10.8, $1.8 \mathrm{~Hz}), 5.82(1 \mathrm{H}, \mathrm{m}), 5.35(1 \mathrm{H}, \mathrm{dd}, J=17.0,1.8 \mathrm{~Hz}), 5.15(1 \mathrm{H}, \mathrm{dd}, J=10.8,1.8 \mathrm{~Hz}), 5.03$ $(1 \mathrm{H}, \mathrm{m}), 4.76(1 \mathrm{H}, \mathrm{s}), 3.93(1 \mathrm{H}, \mathrm{d}, J=4.3 \mathrm{~Hz}), 2.68(1 \mathrm{H}, \mathrm{m}), 2.59(1 \mathrm{H}, \mathrm{dt}, J=12.4,6.8 \mathrm{~Hz}), 2.08(2 \mathrm{H}$, m), $2.00(1 \mathrm{H}, \mathrm{m}), 1.83(2 \mathrm{H}, \mathrm{m}), 1.75(1 \mathrm{H}, \mathrm{dd}, J=13.6,8.4 \mathrm{~Hz}), 1.62(1 \mathrm{H}, \mathrm{br} \mathrm{s}), 1.38(1 \mathrm{H}, \mathrm{ddd}, J=13.0$, 11.4, 7.2 Hz), $1.16(1 \mathrm{H}, \mathrm{dt}, J=12.2,7.8 \mathrm{~Hz}), 1.08(1 \mathrm{H}, \mathrm{ddt}, J=20.0,11.4,8.4 \mathrm{~Hz}), 0.88(9 \mathrm{H}, \mathrm{s}), 0.03$ (3H, s), 0.02 (3H, s); ${ }^{13} \mathrm{C}$ NMR: $\delta 152.3$ (0), 138.8 (1), 127.8 (1), 115.4 (2), 85.5 (0), 83.1 (1), 83.0 (1), 59.5 (0), 50.0 (1), 42.8 (2), 37.1 (2), 36.8 (2), 31.3 (2), 31.2 (2), 25.8 (3C, 3), 18.0 (0), -4.9 (3), -5.1 (3); MS (ESI): $373\left(\mathrm{M}^{+}+\mathrm{Na}\right)$. 
$\left(1 R^{*}, 2 R^{*}, 3 a^{\prime} R^{*}, 5 R^{*}, 5 \mathrm{a}^{\prime} S^{*}\right)-5$-(tert-Butyldimethylsilyl)oxy-2',3',3a',4'-tetrahydro-5'-(methoxymethoxy)-2-vinyl-5' $H$-spiro[cyclopentane-1,1'-pentalen]-2-ol (20)

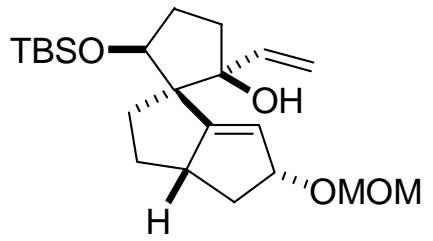

20

$N, N$-Diisopropylethylamine $(13 \mu \mathrm{L}, 0.075 \mathrm{mmol})$ was added to a solution of 19 (15 $\mathrm{mg}, 0.043$ mmol $)$ in $\mathrm{CH}_{2} \mathrm{Cl}_{2}(0.5 \mathrm{~mL})$ and cooled to $0{ }^{\circ} \mathrm{C}$. $\mathrm{MOMCl}(6 \mu \mathrm{L}, 0.075 \mathrm{mmol})$ was added, and the mixture was allowed to warm to rt. The mixture was stirred for $8 \mathrm{~h}$ before addition of saturated aqueous $\mathrm{NaHCO}_{3}$ quenched the reaction. The layers were then separated, and the aqueous layer was extracted with $\mathrm{CH}_{2} \mathrm{CL}_{2}(4 \times 5 \mathrm{~mL})$. The combined organic layers were washed with brine, and then dried over $\mathrm{Na}_{2} \mathrm{SO}_{4}$. The solvent was removed in vacuo, and the residue was purified by flash chromatography (10 \% EtOAc/hexanes) to give 20 (14 mg, 83\%) as a colorless oil. IR ( $\mathrm{KBr}$ disc): 3482 (br) cm ${ }^{-1}$; ${ }^{1} \mathrm{H}$ NMR: $\delta 5.91(1 \mathrm{H}, \mathrm{dd}, J=17.2,10.7 \mathrm{~Hz}), 5.87(1 \mathrm{H}, \mathrm{m}), 5.35(1 \mathrm{H}, \mathrm{dd}, J=17.2,1.8 \mathrm{~Hz}), 5.15(1 \mathrm{H}, \mathrm{dd}, J=10.7$, $1.8 \mathrm{~Hz}), 4.91(1 \mathrm{H}, \mathrm{m}), 4.72(1 \mathrm{H}, \mathrm{s}), 4.70(2 \mathrm{H}, \mathrm{s}), 3.93(1 \mathrm{H}, \mathrm{m}), 3.38(3 \mathrm{H}, \mathrm{s}), 2.65(1 \mathrm{H}, \mathrm{m}), 2.52(1 \mathrm{H}, \mathrm{dt}$, $J=12.1,6.7 \mathrm{~Hz}), 2.08(2 \mathrm{H}, \mathrm{m}), 1.99(1 \mathrm{H}, \mathrm{t}, J=9.2 \mathrm{~Hz}), 1.83(2 \mathrm{H}, \mathrm{m}), 1.76(1 \mathrm{H}, \mathrm{ddd}, J=13.1,8.4,1.2$ $\mathrm{Hz}), 1.36(2 \mathrm{H}, \mathrm{m}), 1.10(1 \mathrm{H}, \mathrm{ddt}, J=19.5,11.0,8.7 \mathrm{~Hz}), 0.87(9 \mathrm{H}, \mathrm{s}), 0.03(3 \mathrm{H}, \mathrm{s}),-0.02(3 \mathrm{H}, \mathrm{s}) ;{ }^{13} \mathrm{C}$ NMR: $\delta 152.0$ (0), 138.8 (1), 125.7 (1), 115.4 (2), 95.8 (2), 88.3 (0), 85.5 (1), 82.9 (1), 59.7 (0), 55.2 (3), 49.2 (1), 39.8 (2), 36.9 (2), 36.6 (2), 31.16 (2), 31.15 (2), 25.7 (3C, 3), 18.0 (0), -4.9 (3), -5.1 (3); MS (ESI): $317\left(\mathrm{M}^{+}+\mathrm{Na}\right)$.

$\left(4 a R^{*}, 7 R^{*}, 7 a R^{*}, 9 a R^{*}\right)-3,4,4 a, 5,6,7,9,9 a-O c t a h y d r o-4 a, 7-d i h y d r o x y-1 H$-cyclopent $[k l]$-as-indacen2(8H)-one

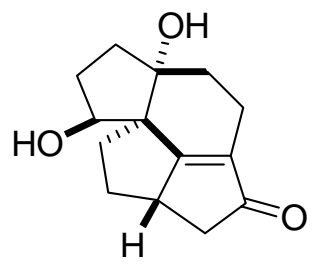

$\mathrm{HF} \bullet$ pyridine $(2.0 \mathrm{~mL})$ was added to a solution of $\mathbf{1 7}(0.303 \mathrm{~g}, 0.870 \mathrm{mmol})$ in $\mathrm{THF}(6.0 \mathrm{~mL})$ at 0 ${ }^{\circ} \mathrm{C}$. The mixture was stirred for $1 \mathrm{~h}$, after which TLC analysis indicated that no starting material remained. The mixture was poured into cold, saturated aqueous $\mathrm{NaHCO}_{3}$, and the mixture was extracted with 1:1 $\mathrm{CHCl}_{3}-\mathrm{CH}_{2} \mathrm{Cl}_{2}(10 \times 25 \mathrm{~mL})$. The combined organic layers were washed with brine and then dried over $\mathrm{Na}_{2} \mathrm{SO}_{4}$. Removal of the solvent in vacuo gave the title compound $(0.187 \mathrm{~g}, 92 \%)$ as a color- 
less crystalline solid. mp: $155-156{ }^{\circ} \mathrm{C}$; IR (KBr disc): 3405 (br), 2942 (s), 1692 (s), 1656 (s) cm ${ }^{-1} ;{ }^{1} \mathrm{H}$ $\operatorname{NMR}\left(\mathrm{CD}_{3} \mathrm{OD}\right): \delta 4.23(1 \mathrm{H}, \mathrm{dd}, J=6.3,4.1 \mathrm{~Hz}), 3.16(1 \mathrm{H}, \mathrm{m}), 2.75(1 \mathrm{H}, \mathrm{dd}, J=18.6,6.0 \mathrm{~Hz}), 2.35$ (1H, ddd, $J=13.4,10.2,3.2 \mathrm{~Hz}), 2.30-2.11(6 \mathrm{H}, \mathrm{m}), 2.04(1 \mathrm{H}, \mathrm{ddd}, J=13.6,8.9,5.0 \mathrm{~Hz}), 1.89-1.73$ (3H, m), 1.59 (1H, m), $1.10(1 \mathrm{H}, \mathrm{m}) ;{ }^{13} \mathrm{C} \mathrm{NMR}\left(\mathrm{CD}_{3} \mathrm{OD}\right): \delta 212.1(0), 188.9$ (0), $137.3(0), 82.0$ (1), 79.9 (0), 59.8 (0), 44.7 (2), 42.0 (1), 38.2 (2), 35.5 (2), 34.9 (2), 32.1 (2), 31.3 (2), 18.5 (2); HRMS: $m / z$ 234.1265 $\left(\mathrm{M}^{+}\right)$, required for $\mathrm{C}_{14} \mathrm{H}_{18} \mathrm{O}_{3}: 234.1256$.

$\left(4 a R^{*}, 7 R^{*}, 7 a R^{*}, 9 a R^{*}\right)-3,4,4 a, 5,6,7,9,9 a-O c t a h y d r o-4 a$-hydroxy-7-methanesulfonyl-1H-cyclopent$[k l]$-as-indacen-2(8H)-one (23)

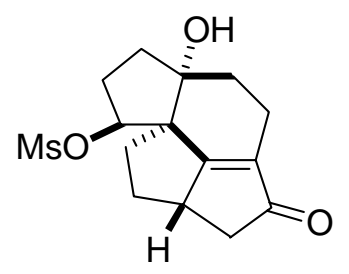

23

Triethylamine $(0.022 \mathrm{~mL}, 0.16 \mathrm{mmol})$ was added to a solution of $(4 a \mathrm{R} *, 7 \mathrm{R} *, 7 a \mathrm{R} *, 9 a \mathrm{R} *)$ 3,4,4a,5,6,7,9,9a-octahydro-4a,7-dihydroxy-1H-cyclopent[k1]-as-indacen-2(8H)-one (0.163 g, 0.694 mmol) in $\mathrm{CH}_{2} \mathrm{Cl}_{2}$. Mesyl chloride (60 $\mu \mathrm{L}, 0.76 \mathrm{mmol}$ ) was added, and the mixture was warmed to rt. After $3 \mathrm{~h}$ the reaction was quenched with saturated aqueous $\mathrm{NaHCO}_{3}$. The solution was extracted with $\mathrm{CH}_{2} \mathrm{Cl}_{2}(4 \times 5 \mathrm{~mL})$. The combined organic layers were washed with brine, and then dried over $\mathrm{Na}_{2} \mathrm{SO}_{4}$. Evaporation of solvent in vacuo followed by flash chromatography (75\% EtOAc/hexanes) yielded $\mathbf{2 3}$ (0.154 g, 71\%) as a white powder. mp: 132-134 ${ }^{\circ} \mathrm{C}$; IR (KBr disc): 3425 (br), 2938 (s), 1682 (s), 1653 (s), $1385(\mathrm{~s}) \mathrm{cm}^{-1}$; ${ }^{1} \mathrm{H}$ NMR: $\delta 5.09(1 \mathrm{H}, \mathrm{dd}, J=6.6,2.9 \mathrm{~Hz}), 3.13(1 \mathrm{H}, \mathrm{m}), 2.94(3 \mathrm{H}, \mathrm{s}), 2.80(1 \mathrm{H}, \mathrm{dd}, J$ = 18.6, 6.1 Hz), 2.48-2.34 (2H, m), 2.33-2.22 (3H, m), 2.19-2.04 (3H, m), 2.00-1.90 (2H, m), 1.82 (2H, m), $1.31(1 \mathrm{H}, \mathrm{s}), 1.07(1 \mathrm{H}, \mathrm{m}) ;{ }^{13} \mathrm{C} \mathrm{NMR}: \delta 208.6(0), 181.3(0), 136.5(0), 88.0(1), 78.6(0), 56.9$ (0), 43.7 (2), 40.2 (1), 38.6 (3), 36.6 (2), 33.9 (2), 33.7 (2), 30.4(2), 29.4 (2), 17.2 (2); HRMS: m/z $312.1029\left(\mathrm{M}^{+}\right)$, required for $\mathrm{C}_{15} \mathrm{H}_{20} \mathrm{O}_{5} \mathrm{~S}: 312.1031$. 
$\left(2 R^{*}, 4 a S^{*}, 7 S^{*}, 7 a S^{*}, 9 a S^{*}\right)-2,3,4,4 a, 5,6,7,8,9,9 a-D e c a h y d r o-7-m e t h a n e s u l f o n y l-1 H$-cyclopent $[k l]$-asindacene-2,4a-diol (24)

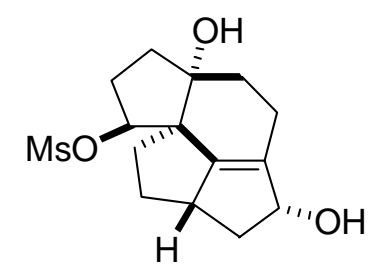

24

$\mathrm{CeCl}_{3} \cdot 7 \mathrm{H}_{2} \mathrm{O}(133 \mathrm{mg}, 0.356 \mathrm{mmol})$ was added to a solution of $23(9.3 \mathrm{mg}, 0.30 \mathrm{mmol})$ in methanol $(4.0 \mathrm{~mL})$ at $0^{\circ} \mathrm{C}$. The solution was then treated with $\mathrm{NaBH}_{4}(9 \mathrm{mg}, 0.02 \mathrm{mmol})$. After stirring the mixture for $30 \mathrm{~min}$ at $0^{\circ} \mathrm{C}$, TLC analysis indicated that the reaction was complete. The reaction was quenched with saturated aqueous $\mathrm{NH}_{4} \mathrm{Cl}$. The mixture was extracted with $\mathrm{CH}_{2} \mathrm{Cl}_{2}(5 \times 5 \mathrm{~mL})$. The combined organic layers were washed with brine and then dried over $\mathrm{Na}_{2} \mathrm{SO}_{4}$. The solvent was removed in vacuo to give 24 as a colorless crystalline solid (9.2 mg, 97\%). mp: 92-94 ${ }^{\circ} \mathrm{C}$ (dec.); IR (KBr disc): 3356 (br), 2935 (s), 1446 (s), 1344 (s) cm ${ }^{-1}$; ${ }^{1} \mathrm{H}$ NMR: $\delta 5.04$ (1H, m), 4.89 (1H, m), 2.90 (3H, s), 2.84 (1H, m), $2.81(1 \mathrm{H}, \mathrm{m}), 2.40-2.25(3 \mathrm{H}, \mathrm{m}), 2.18(1 \mathrm{H}, \mathrm{m}), 2.11-1.97(4 \mathrm{H}, \mathrm{m}), 1.93-1.78(3 \mathrm{H}, \mathrm{m}), 1.54$ (2H, m), 1.43 (1H, m), $1.20(1 \mathrm{H}, \mathrm{m}) ;{ }^{13} \mathrm{C}$ NMR: $\delta 146.7$ (0), 137.2 (0), 88.8 (1), $81.2(1), 78.4$ (0), 55.8 (0), 44.6 (2), 44.3 (1), 38.5 (3), 36.2 (2), 34.5 (2), 33.4 (2), 31.3 (2), 29.3 (2), 18.8 (2); MS (ESI): 337 $\left(\mathrm{M}^{+}+\mathrm{Na}\right)$.

$\left(2 a R^{*}, 4 S^{*}\right)-2,2 a, 3,4,5,6,8,9-O c t a h y d r o-4-h y d r o x y c y c l o n o n a[c d] p e n t a l e n-7(1 H)-o n e ~(25)$

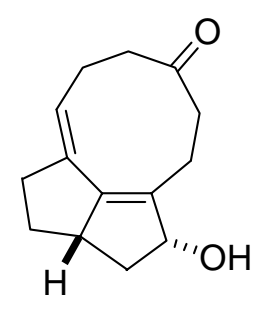

25

To a solution of $24(21 \mathrm{mg}, 0.067 \mathrm{mmol})$ in $t-\mathrm{BuOH}(1.0 \mathrm{~mL})$ at $\mathrm{rt}$ was added $\mathrm{KO} t \mathrm{Bu}(15 \mathrm{mg}$, $0.13 \mathrm{mmol})$ followed by 18 -crown-6 $(38 \mathrm{mg}, 0.15 \mathrm{mmol})$. The mixture was stirred for $5 \mathrm{~h}$ after which time TLC indicated that no starting material remained. The reaction was then quenched with saturated aqueous $\mathrm{NH}_{4} \mathrm{Cl}$, and the mixture was extracted with $\mathrm{CH}_{2} \mathrm{Cl}_{2}(4 \times 10 \mathrm{~mL})$. The combined organic layers were washed with brine and dried over $\mathrm{Na}_{2} \mathrm{SO}_{4}$. Evaporation of the solvent in vacuo followed by flash chromatography (8\% EtOAc/hexanes) afforded $25(7 \mathrm{mg}, 45 \%)$ as a white solid. $\mathrm{mp}: 94-96{ }^{\circ} \mathrm{C}$; IR (KBr disc): 3404 (br), 2950 (s), 2859 (m), 1694 (s), 1435 (m) cm ${ }^{-1} ;{ }^{1} \mathrm{H}$ NMR: $\delta 5.40$ (1H, m), 4.85 (1H, 
m), 3.26-3.09 (2H, m), 2.95 (1H, dt, $J=12.4,4.9 \mathrm{~Hz}), 2.76-2.38$ (10H, m), 1.91 (1H, m), 1.21-1.08 (2H, m); ${ }^{13} \mathrm{C}$ NMR: $\delta 213.3$ (0), 144.8 (0), 138.3 (0), 134.7 (0), 121.4 (1), 85.0 (1), 49.2 (1), 43.5 (2), 43.2 (2), 43.1 (2), 35.2 (2), 31.1 (2), 27.1 (2), 22.1 (2). MS: $m / z 218.1315\left(\mathrm{M}^{+}\right)$, required for $\mathrm{C}_{14} \mathrm{H}_{18} \mathrm{O}_{2}$ : 218.1307.

$\left(2 R^{*}, 4 a S^{*}, 7 S^{*}, 7 a S^{*}, 9 a S^{*}\right)-2,3,4,4 a, 5,6,7,8,9,9 a$-Decahydro-7-methanesulfonyl-2-(methoxymethyl)$1 H$-cyclopent $[k l]$-as-indacen-4a-ol (26)

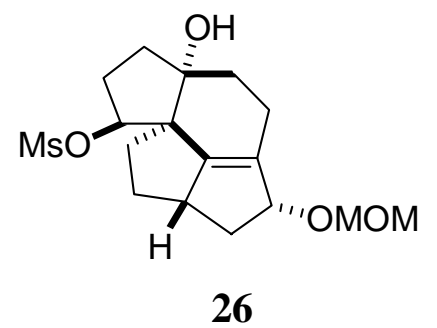

$N, N$-Diisopropylethylamine (31 mg, $0.24 \mathrm{mmol}$ ) was added to a solution of 24 (50 $\mathrm{mg}, 0.16$ mmol) in $\mathrm{CH}_{2} \mathrm{Cl}_{2}(1.6 \mathrm{~mL})$ at $0{ }^{\circ} \mathrm{C}$. MOMCl $(19 \mathrm{mg}, 0.24 \mathrm{mmol})$ was added, and the mixture was allowed to attain rt. After $4 \mathrm{~h}$, TLC analysis indicated that no starting material remained. The reaction was quenched with saturated aqueous $\mathrm{NaHCO}_{3}$, and the solution was extracted with $\mathrm{CH}_{2} \mathrm{Cl}_{2}(4 \times 5 \mathrm{~mL})$. The combined organic layers were washed with brine and then dried over $\mathrm{Na}_{2} \mathrm{SO}_{4}$. Removal of solvent in vacuo followed by flash chromatography (20\% EtOAc/hexanes) afforded 26 (46 mg, 81\%) as a colorless oil. IR (KBr disc): 3448 (br), 2942 (s), 1443 (m), 1351 (s) cm ${ }^{-1} ;{ }^{1} \mathrm{H}$ NMR: $\delta 4.94(1 \mathrm{H}, \mathrm{m}), 4.91$ $(1 \mathrm{H}, \mathrm{m}), 4.68(1 \mathrm{H}, \mathrm{d}, J=6.7 \mathrm{~Hz}), 4.66(1 \mathrm{H}, \mathrm{d}, J=6.7 \mathrm{~Hz}), 3.38(3 \mathrm{H}, \mathrm{s}), 2.90(3 \mathrm{H}, \mathrm{s}), 2.85(1 \mathrm{H}, \mathrm{m}), 2.76$ (1H, m), 2.39-2.25 (2H, m), 2.18 (1H, m), 2.11-1.98 (4H, m), 1.93-1.79 (3H, m), 1.55 (2H, m), 1.18 (1H, m); ${ }^{13} \mathrm{C}$ NMR: $\delta 147.0(0), 136.0$ (0), 95.8 (2), 88.7 (1), 86.4 (1), 78.4 (0), 55.8 (0), 55.4 (3), 44.2 (1), 42.2 (2), 38.5 (3), 35.9 (2), 34.5 (2), 33.2 (2), 31.1 (2), 29.3 (2), 19.1 (2); MS (ESI): $381\left(\mathrm{M}^{+}+\mathrm{Na}\right)$.

$\left(2 a R *, 4 S^{*}\right)-2,2 a, 3,4,5,6,8,9-O c t a h y d r o-4-(m e t h o x y m e t h o x y) c y c l o n o n a[c d] p e n t a l e n-7(1 H)-o n e ~(27)$

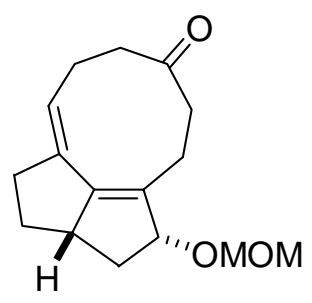

27

To a solution of $26(10 \mathrm{mg}, 0.027 \mathrm{mmol})$ in $t$-BuOH $(0.3 \mathrm{~mL})$ at $\mathrm{rt}$ was added $\mathrm{KO} t \mathrm{Bu}(4 \mathrm{mg}$, $0.04 \mathrm{mmol})$ followed by 18 -crown-6 $(8 \mathrm{mg}, 0.03 \mathrm{mmol})$. The mixture was stirred for $4 \mathrm{~h}$ after which 
time TLC analysis showed that the no starting material remained. The reaction was then quenched with saturated aqueous $\mathrm{NH}_{4} \mathrm{Cl}$, and the mixture was extracted with $\mathrm{CH}_{2} \mathrm{Cl}_{2}(4 \times 10 \mathrm{~mL})$. The combined organic layers were washed with brine and then dried over $\mathrm{Na}_{2} \mathrm{SO}_{4}$. Evaporation of the solvent in vacuo followed by flash chromatography (5\% EtOAc/hexanes) gave 27 (5 mg, 81\%) as a colorless oil. IR (KBr disc): 2949 (s), 1702 (s), 1441 (m); ${ }^{1} \mathrm{H}$ NMR: $\delta 5.41(1 \mathrm{H}, \mathrm{m}), 4.77(1 \mathrm{H}, \mathrm{m}), 4.71(1 \mathrm{H}, \mathrm{d}, J=7.0$ Hz), $4.66(1 \mathrm{H}, \mathrm{d}, J=7.0 \mathrm{~Hz}), 3.38(3 \mathrm{H}, \mathrm{s}), 3.08(2 \mathrm{H}, \mathrm{m}), 2.94(1 \mathrm{H}, \mathrm{m}), 2.77-2.35(9 \mathrm{H}, \mathrm{m}), 1.91(1 \mathrm{H}$, m), 1.35 (1H, m), $1.16(1 \mathrm{H}, \mathrm{m}) ;{ }^{13} \mathrm{C}$ NMR: $\delta 213.3$ (0), $144.6(0), 138.2(0), 133.5(0), 121.5$ (1), 96.2 (2), 90.3 (1), 55.5 (3), 49.3 (1), 42.9 (2), 42.7 (2), 40.7 (2), 35.0 (2), 30.9 (2), 27.0 (2), 22.2 (2); HRMS: $m / z, 262.1567\left(\mathrm{M}^{+}\right)$, required for $\mathrm{C}_{16} \mathrm{H}_{22} \mathrm{O}_{3}: 262.1569$.

\section{$\left(2 R^{*}, 3 S^{*}\right)$-2-(But-3-enyl)-3-(tert-butyldimethylsilyl)oxy-2-((trimethylsilyl)ethynyl)cyclopentanone}

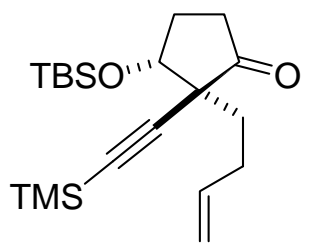

Triethylamine $(0.175 \mathrm{~mL}, 1.20 \mathrm{mmol})$ was added to a solution of $\mathbf{1 3}(0.282 \mathrm{~g}, 1.11 \mathrm{mmol})$ in THF (10 mL) and cooled to $0{ }^{\circ} \mathrm{C}$. TBSOTf $(0.22 \mathrm{~mL}, 9.1 \mathrm{mmol})$ was added dropwise to the solution. The mixture was stirred for $30 \mathrm{~min}$ at $0{ }^{\circ} \mathrm{C}$, after which time TLC analysis indicated that no alcohol remained. The reaction was quenched with excess saturated aqueous $\mathrm{NaHCO}_{3}$, and the layers were separated. The aqueous layer was extracted with $\mathrm{CH}_{2} \mathrm{Cl}_{2}(4 \times 5 \mathrm{~mL})$. The combined organic layers were washed with brine and dried over $\mathrm{MgSO}_{4}$. Removal of the solvent in vacuo and purification of the residue using flash chromatography (3\% EtOAc/hexanes) yielded the title compound (0.390 g, 96\%) as a colorless oil. IR $\left(\mathrm{CH}_{2} \mathrm{Cl}_{2}\right): 3054(\mathrm{~s}), 2986(\mathrm{~s}), 2306(\mathrm{~m}), 1750(\mathrm{~s}) \mathrm{cm}^{-1} ;{ }^{1} \mathrm{H}$ NMR: $\delta 5.85(1 \mathrm{H}, \mathrm{ddt}, J=$ 16.8, 10.2, $6.7 \mathrm{~Hz}), 5.04(1 \mathrm{H}, \mathrm{dq}, J=17.2,1.7 \mathrm{~Hz}), 4.96(1 \mathrm{H}, \mathrm{dd}, J=10.2,1.8 \mathrm{~Hz}), 4.35(1 \mathrm{H}, \mathrm{m}), 2.48-$ $2.31(4 \mathrm{H}, \mathrm{m}), 2.09(1 \mathrm{H}, \mathrm{m}), 1.91(1 \mathrm{H}, \mathrm{m}), 1.75(1 \mathrm{H}, \mathrm{dt}, J=13.4,4.8 \mathrm{~Hz}), 1.67$ (1H, ddd, $J=13.4,12.1$, $4.8 \mathrm{~Hz}), 0.86(9 \mathrm{H}, \mathrm{s}), 0.13(9 \mathrm{H}, \mathrm{s}), 0.11(3 \mathrm{H}, \mathrm{s}), 0.10(3 \mathrm{H}, \mathrm{s}) ;{ }^{13} \mathrm{C}$ NMR: $\delta 212.6(0), 138.4(1), 114.5$ (2), 102.9(0), 90.9 (0), 76.6 (1), 55.7 (0), 33.2 (2), 29.1 (2), 28.9 (2), 28.0 (2), 25.6 (3C, 3$), 18.3$ (0), -2.9 (3C, 3), -4.4 (3), -4.9 (3); HRMS: $m / z, 364.2265\left(\mathrm{M}^{+}\right)$, required for $\mathrm{C}_{20} \mathrm{H}_{36} \mathrm{O}_{2} \mathrm{Si}_{2}: 364.2254$. 


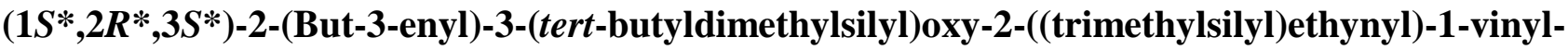
cyclopentan-1-ol

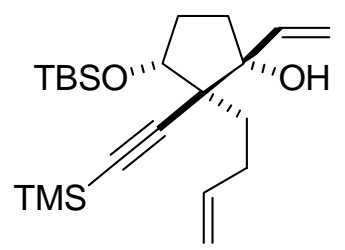

A $1 \mathrm{M}$ solution of vinylmagnesium bromide in $\mathrm{THF}(1.1 \mathrm{~mL}, 1.1 \mathrm{mmol})$ was added to a suspension of anhydrous $\mathrm{CeCl}_{3}(0.270 \mathrm{~g}, 12.0 \mathrm{mmol})$ stirring in $\mathrm{THF}(3.0 \mathrm{~mL})$ at $-78{ }^{\circ} \mathrm{C}$. The resulting suspension was stirred at $-78{ }^{\circ} \mathrm{C}$ for $2 \mathrm{~h}$. A solution of (2R*,3S*)-2-(but-3-enyl)-3-(tertbutyldimethylsilyl)oxy-2-((trimethylsilyl)ethynyl)cyclopentanone $(0.200 \mathrm{~g}, 0.548 \mathrm{mmol})$ in THF (1.0 $\mathrm{mL}$ ) was added, and the mixture was stirred at $-78^{\circ} \mathrm{C}$ for $1 \mathrm{~h}$. After this time, TLC analysis indicated that no starting material remained. The mixture was then warmed to $\mathrm{rt}$, and the reaction was quenched with $5 \%$ aqueous $\mathrm{AcOH}(1.0 \mathrm{~mL})$. The layers were separated, and the aqueous layer was extracted with $\mathrm{Et}_{2} \mathrm{O}(4 \times 20 \mathrm{~mL})$. The combined organic layers were washed with saturated aqueous $\mathrm{NaHCO}_{3}$ and brine, and then dried over $\mathrm{Na}_{2} \mathrm{SO}_{4}$. The solvent was evaporated under reduced pressure, and the residue was purified by flash chromatography (2\% EtOAc/hexanes) to give the title compound $(0.195 \mathrm{~g}, 91 \%)$ as a colorless oil. IR $\left(\mathrm{CH}_{2} \mathrm{Cl}_{2}\right): 3476(\mathrm{br}), 3054(\mathrm{~s}), 2986(\mathrm{~s}), 2305(\mathrm{~m}), 1421(\mathrm{~s}) \mathrm{cm}^{-1} ;{ }^{1} \mathrm{H}$ NMR: $\delta 6.14$ $(1 \mathrm{H}, \mathrm{ddd}, J=17.2,10.8,2.0 \mathrm{~Hz}), 5.84(1 \mathrm{H}, \mathrm{ddt}, J=17.0,10.2,6.6 \mathrm{~Hz}), 5.40(1 \mathrm{H}, \mathrm{dd}, J=17.0,2.0 \mathrm{~Hz})$, $5.17(1 \mathrm{H}, \mathrm{dd}, J=10.8,2.0 \mathrm{~Hz}), 5.02(1 \mathrm{H}, \mathrm{dq}, J=17.0,1.8 \mathrm{~Hz}), 4.93(1 \mathrm{H}, \mathrm{dd}, J=10.2,1.8 \mathrm{~Hz}), 4.27$ $(1 \mathrm{H}, \mathrm{d}, J=4.9 \mathrm{~Hz}), 4.09(1 \mathrm{H}, \mathrm{d}, J=1.7 \mathrm{~Hz}), 2.41-2.26(3 \mathrm{H}, \mathrm{m}), 2.03(1 \mathrm{H}, \mathrm{m}), 1.93(1 \mathrm{H}, \mathrm{m}), 1.80(2 \mathrm{H}$, m), $1.50(1 \mathrm{H}, \mathrm{ddd}, J=18.0,13.1,4.9 \mathrm{~Hz}), 0.90(9 \mathrm{H}, \mathrm{s}), 0.14(9 \mathrm{H}, \mathrm{s}), 0.14(3 \mathrm{H}, \mathrm{s}), 0.12(3 \mathrm{H}, \mathrm{s}) ;{ }^{13} \mathrm{C}$ NMR: $\delta 140.2$ (1), 138.9 (1), 114.1 (2), 114.1 (2), 107.8 (0), 90.4 (0), 85.1 (0), 80.5 (0), 55.7 (0), 38.6 (2), 32.2 (2), 29.9 (2), 26.6 (2), 25.7 (3C, 3), 17.8 (0), 0.0 (3C, 3), -4.5 (3), -5.1 (3); HRMS: m/z $392.2577\left(\mathrm{M}^{+}\right)$, required for $\mathrm{C}_{22} \mathrm{H}_{40} \mathrm{O}_{2} \mathrm{Si}_{2}: 392.2567$.

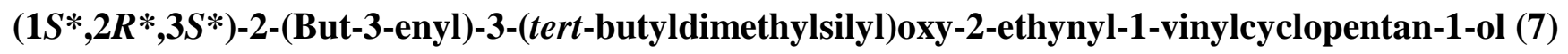

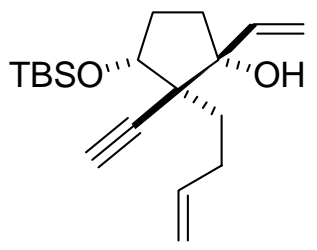

7

Anhydrous $\mathrm{K}_{2} \mathrm{CO}_{3}(75 \mathrm{mg}, 0.54 \mathrm{mmol})$ was added to a solution of $(1 \mathrm{~S} *, 2 \mathrm{R} *, 3 \mathrm{~S} *)-2-($ but-3enyl)-3-(tert-butyldimethylsilyl)oxy-2-((trimethylsilyl)ethynyl)-1-vinyl-cyclopentan-1-ol (0.164 g, 0.417 $\mathrm{mmol})$ in methanol $(2.5 \mathrm{~mL})$ at $0{ }^{\circ} \mathrm{C}$. The mixture was warmed to $\mathrm{rt}$ and stirred for $8 \mathrm{~h}$. The mixture was 
diluted with $\mathrm{H}_{2} \mathrm{O}$ and $\mathrm{Et}_{2} \mathrm{O}$, and the layers were separated. The aqueous layer was extracted with $\mathrm{Et}_{2} \mathrm{O}(5$ $\times 10 \mathrm{~mL}$ ). The combined organic layers were washed with brine and dried over $\mathrm{Na}_{2} \mathrm{SO}_{4}$. The solvent was removed under vacuum, and the residue was purified using flash chromatography (5\% EtOAc/hexanes) to give $7(0.128 \mathrm{~g}, 96 \%)$ as a colorless oil. IR $\left(\mathrm{CH}_{2} \mathrm{Cl}_{2}\right): 3503$ (br), 3308 (w), 2931 (m) $\mathrm{cm}^{-1} ;{ }^{1} \mathrm{H}$ NMR: $\delta 6.15(1 \mathrm{H}, \mathrm{ddd}, J=17.3,10.8,2.0 \mathrm{~Hz}), 5.83(1 \mathrm{H}, \mathrm{ddt}, J=17.0,10.3,6.6 \mathrm{~Hz}), 5.42$ $(1 \mathrm{H}, \mathrm{dd}, J=17.3,2.0 \mathrm{~Hz}), 5.19(1 \mathrm{H}, \mathrm{dd}, J=10.8,2.0 \mathrm{~Hz}), 5.03(1 \mathrm{H}, \mathrm{dd}, J=17.0,1.5 \mathrm{~Hz}), 4.94(1 \mathrm{H}, \mathrm{dd}$, $J=10.3,1.5 \mathrm{~Hz}), 4.31(1 \mathrm{H}, \mathrm{d}, J=4.6 \mathrm{~Hz}), 4.06(1 \mathrm{H}, \mathrm{d}, J=1.5 \mathrm{~Hz}), 2.43-2.28(3 \mathrm{H}, \mathrm{m}), 2.25(1 \mathrm{H}, \mathrm{s})$, $2.05(1 \mathrm{H}, \mathrm{m}), 1.96(1 \mathrm{H}, \mathrm{m}), 1.83(2 \mathrm{H}, \mathrm{m}), 1.53(1 \mathrm{H}, \mathrm{ddd}, J=18.0,13.3,4.6 \mathrm{~Hz}), 0.91(9 \mathrm{H}, \mathrm{s}), 0.14(3 \mathrm{H}$, s), 0.12 (3H, s); ${ }^{13} \mathrm{C}$ NMR: $\delta 139.9$ (1), 138.7 (1), 114.4 (2), 114.3 (2), 85.3 (0), 84.9 (0), 80.5 (1), 73.8 (0), 54.7 (0), 38.5 (2), 32.1 (2), 29.7 (2), $26.6(2), 25.7$ (3C, 3), 17.8 (0), -4.4 (3), -5.2 (3).

$\left(1 R^{*}, 2 S^{*}, 3 a^{\prime} R^{*}, 5 S^{*}, 5 \mathrm{a}^{\prime} S^{*}\right)-(28)$ and $\left(1 R^{*}, 2 S^{*}, 3 a^{\prime} S^{*}, 5 S^{*}, 5 \mathrm{a}^{\prime} R^{*}\right)-5$-(tert-Butyldimethylsilyl)oxy2',3',3a',4'-tetrahydro-2-vinyl-5' $H$-spiro[cyclopentane-1,1'-pentalene]-2,5'-diol (29)

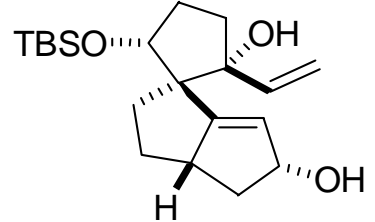

28

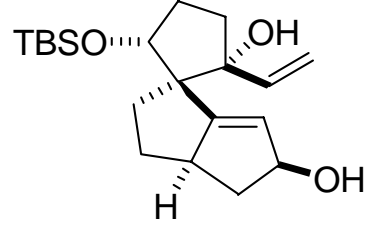

29

Dicobalt octacarbonyl (135 mg, $0.395 \mathrm{mmol})$ was added to a solution of 7 (110 $\mathrm{mg}, 0.343 \mathrm{mmol})$ in $\mathrm{CH}_{2} \mathrm{Cl}_{2}(1.8 \mathrm{~mL})$. The resulting red solution was stirred at $\mathrm{rt}$ for $6 \mathrm{~h}$, after which time TLC analysis indicated that no alcohol remained. The mixture was diluted with $\mathrm{CH}_{2} \mathrm{Cl}_{2}(40 \mathrm{~mL})$ and treated with a solution of anhydrous TMANO ${ }^{\dagger}(185 \mathrm{mg}, 0.246 \mathrm{mmol})$ in $\mathrm{CH}_{2} \mathrm{Cl}_{2}(28 \mathrm{~mL})$. The mixture was stirred for $14 \mathrm{~h}$ at $\mathrm{rt}$, during which time a deep blue precipitate formed. The mixture was filtered through a plug of silica gel (EtOAc eluent) and then concentrated under reduced pressure to give a mixture of diastereomeric enones (0.108 g, 90\%, $d r$ 4:1 by ${ }^{1} \mathrm{H}$ NMR), which were inseparable by flash chromatography. These diastereomers were dissolved in $\mathrm{MeOH}(2.0 \mathrm{~mL})$ and cooled to $0{ }^{\circ} \mathrm{C}$. $\mathrm{CeCl}_{3} \cdot 7 \mathrm{H}_{2} \mathrm{O}(0.191 \mathrm{~g}, 0.515 \mathrm{~mol})$ was added to the solution followed by $\mathrm{NaBH}_{4}(6.0 \mathrm{mg}, 0.16 \mathrm{mmol})$. The mixture was stirred at $0{ }^{\circ} \mathrm{C}$ for $1 \mathrm{~h}$, and the reaction was quenched with saturated aqueous $\mathrm{NH}_{4} \mathrm{Cl}(1$ $\mathrm{mL})$. The mixture extracted with $\mathrm{CH}_{2} \mathrm{Cl}_{2}(4 \times 5 \mathrm{~mL})$. The organic layers were combined, washed with brine, and then dried over $\mathrm{Na}_{2} \mathrm{SO}_{4}$. Removal of the solvent in vacuo and flash chromatography (15\% EtOAc/hexanes) gave $\mathbf{2 8}$ (85 mg, 71\% for two steps) and $\mathbf{2 9}$ (8 mg, 6\% for two steps) as colorless oils.

\footnotetext{
${ }^{\dagger}$ Anhydrous TMANO was obtained from TMANO dihydrate by azeotropic distillation in benzene.
} 
For 28: IR (KBr disc): 3489 (br s), 2953 (s), 2859 (s), 1653 (w) cm ${ }^{-1} ;{ }^{1} \mathrm{H}$ NMR: $\delta 5.94$ (1H, ddd, $J=17.1,10.8,1.6 \mathrm{~Hz}), 5.42(1 \mathrm{H}, \mathrm{dd}, J=17.1,2.3 \mathrm{~Hz}), 5.23(1 \mathrm{H}, \mathrm{m}), 5.09(1 \mathrm{H}, \mathrm{dd}, J=10.8,2.3 \mathrm{~Hz})$, $5.03(1 \mathrm{H}, \mathrm{m}), 4.52(1 \mathrm{H}, \mathrm{d}, J=1.4 \mathrm{~Hz}), 3.87(1 \mathrm{H}, \mathrm{d}, J=5.0 \mathrm{~Hz}), 2.73(1 \mathrm{H}, \mathrm{m}), 2.57(1 \mathrm{H}, \mathrm{dt}, J=12.1,6.6$ Hz), 2.28 (1H, dd, J = 14.0, 7.9 Hz), 2.20 (2H, m), 2.10 (1H, m), 1.94 (1H, m), 1.90-1.79 (3H, m), 1.61 (1H, br s) $1.21(1 \mathrm{H}, \mathrm{ddd}, J=16.5,12.1,8.1 \mathrm{~Hz}), 1.11(1 \mathrm{H}, \mathrm{ddt}, J=19.8,8.1,11.6 \mathrm{~Hz}), 0.90(9 \mathrm{H}, \mathrm{s})$, $0.09(3 \mathrm{H}, \mathrm{s}), 0.09(3 \mathrm{H}, \mathrm{s}) ;{ }^{13} \mathrm{C}$ NMR: $\delta 156.1(0), 139.8(1), 124.3(1), 114.0(2), 84.2(0), 83.0(1), 82.3$ (1), 61.5 (0), 50.8 (1), 43.0 (2), 38.0 (2), 32.1 (2), 31.1 (2), 30.1 (2), 25.7 (3C, 3), 17.9 (0), -4.7 (3), -5.1 (3); $\mathrm{MS}(\mathrm{ESI}): 373\left(\mathrm{M}^{+}+\mathrm{Na}\right)$.

For 29: IR (KBr disc): 3498 (br s), 2954 (s), 2859 (s), 1647 (w) cm ${ }^{-1} ;{ }^{1} \mathrm{H}$ NMR: $\delta 5.56$ (1H, ddd, $J=17.1,10.7,1.7 \mathrm{~Hz}), 5.39(1 \mathrm{H}, \mathrm{dd}, J=17.1,2.3 \mathrm{~Hz}), 5.21(1 \mathrm{H}, \mathrm{m}), 5.12(1 \mathrm{H}, \mathrm{dd}, J=10.7,2.3 \mathrm{~Hz})$, $5.09(1 \mathrm{H}, \mathrm{m}), 4.14(1 \mathrm{H}, \mathrm{d}, J=1.7 \mathrm{~Hz}), 4.04(1 \mathrm{H}, \mathrm{d}, J=5.5 \mathrm{~Hz}), 2.67(1 \mathrm{H}, \mathrm{m}), 2.55(1 \mathrm{H}, \mathrm{dt}, J=12.2,6.6$ Hz), $2.31(1 \mathrm{H}, \mathrm{m}), 2.15(1 \mathrm{H}, \mathrm{dd}, J=13.3,7.6 \mathrm{~Hz}), 2.08(1 \mathrm{H}, \mathrm{ddd}, J=18.0,12.2,6.6 \mathrm{~Hz}), 2.03-1.83$ $(4 \mathrm{H}, \mathrm{m}), 1.19(1 \mathrm{H}, \mathrm{dt}, J=12.2,8.1 \mathrm{~Hz}), 1.03(1 \mathrm{H}, \mathrm{ddt}, J=17.7,6.1,10.5 \mathrm{~Hz}), 0.91(9 \mathrm{H}, \mathrm{s}), 0.10(3 \mathrm{H}, \mathrm{s})$, 0.09 (3H, s); ${ }^{13} \mathrm{C}$ NMR: $\delta 158.1(0), 139.9$ (1), 123.1 (1), 114.8 (2), $85.2(0), 82.7$ (1), $80.9(1), 61.4(0)$, 51.7 (1), 42.9 (2), 37.3 (2), 32.7 (2), 31.5 (2), 29.2 (2), 25.8 (3C, 3), 17.9 (0), -4.8 (3), -5.1 (3); HRMS: $m / z 350.2262\left(\mathrm{M}^{+}\right)$, required for $\mathrm{C}_{20} \mathrm{H}_{34} \mathrm{O}_{3} \mathrm{Si}: 350.2277$.

\section{$\left(1 R^{*}, 2 S^{*}, 3 a^{\prime} R^{*}, 5 S^{*}, 5 \mathrm{a}^{\prime} S^{*}\right)-5$-(tert-Butyldimethylsilyl)oxy-2',3',3a',4'-tetrahydro-5' -(methoxy-} methoxy)-2-vinyl-5' $H$-spiro[cyclopentane-1,1'-pentalen]-2-ol (30)

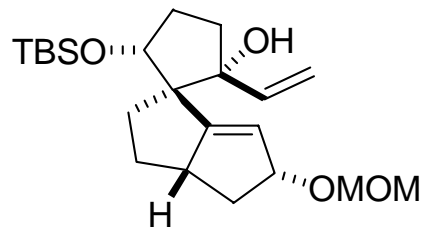

30

$N, N$-Diisopropylethylamine $(56 \mu \mathrm{L}, 0.32 \mathrm{mmol})$ was added to a solution of $28(93 \mathrm{mg}, 0.26$ mmol) in $\mathrm{CH}_{2} \mathrm{Cl}_{2}(2.0 \mathrm{~mL})$ and cooled to $0{ }^{\circ} \mathrm{C} . \mathrm{MOMCl}(24 \mu \mathrm{L}, 0.32 \mathrm{mmol})$ was added, and the mixture was allowed to warm to $\mathrm{rt}$. After stirring for $7 \mathrm{~h}$, TLC analysis indicated that none of the starting material remained. The reaction was quenched by addition of saturated aqueous $\mathrm{NaHCO}_{3}$, and the layers were separated. The aqueous layer was extracted with $\mathrm{CH}_{2} \mathrm{Cl}_{2}(4 \times 5 \mathrm{~mL})$. The combined organic layers were washed with brine and then dried over $\mathrm{Na}_{2} \mathrm{SO}_{4}$. The solvent was removed in vacuo, and the residue was purified by flash chromatography (8\% EtOAc/hexanes) to provide 30 (103 $\mathrm{mg}, 82 \%)$ as a colorless oil. IR (KBr disc): 3492 (br m), 2953 (s), 1646 (w) cm ${ }^{-1} ;{ }^{1} \mathrm{H}$ NMR: $\delta 5.97$ (1H, ddd, $J=17.1,10.8,1.6$ $\mathrm{Hz}), 5.41(1 \mathrm{H}, \mathrm{dd}, J=17.1,1.6 \mathrm{~Hz}), 5.27(1 \mathrm{H}, \mathrm{br} \mathrm{s}), 5.08(1 \mathrm{H}, \mathrm{dd}, J=10.8,1.6 \mathrm{~Hz}), 4.87(1 \mathrm{H}, \mathrm{m}), 4.70$ 
$(1 \mathrm{H}, \mathrm{d}, J=6.7 \mathrm{~Hz}), 4.68(1 \mathrm{H}, \mathrm{d}, J=6.7 \mathrm{~Hz}), 4.51(1 \mathrm{H}, \mathrm{s}), 3.86(1 \mathrm{H}, \mathrm{d}, J=4.7 \mathrm{~Hz}), 3.38(3 \mathrm{H}, \mathrm{s}), 2.70$ $(1 \mathrm{H}, \mathrm{m}), 2.50(1 \mathrm{H}, \mathrm{dt}, J=14.0,8.2 \mathrm{~Hz}), 2.28(1 \mathrm{H}, \mathrm{dd}, J=14.0,8.2 \mathrm{~Hz}), 2.20(2 \mathrm{H}, \mathrm{m}), 2.09(1 \mathrm{H}, \mathrm{t}, J=$ $9.5 \mathrm{~Hz}), 1.98-1.78(3 \mathrm{H}, \mathrm{m}), 1.38(1 \mathrm{H}, \mathrm{dt}, J=12.0,8.6 \mathrm{~Hz}), 1.12(1 \mathrm{H}, \mathrm{ddt}, J=19.8,12.0,8.6 \mathrm{~Hz}), 0.9$ (9H, s), 0.09 (6H, s); ${ }^{13} \mathrm{C}$ NMR: $\delta 155.9$ (0), 139.9 (1), 122.5 (1), 113.9 (2), 96.1 (2), 88.8 (1), 84.2 (0), 82.3 (1), 61.6 (0), 55.3 (3), 50.18 (1), 40.0 (2), 38.2 (2), 32.1 (2), 31.0 (2), 30.2 (2), 25.7 (3, 3C), 17.9 (0), -4.7 (3), -5.1 (3); HRMS: $m / z 394.2539\left(\mathrm{M}^{+}\right)$, required for $\mathrm{C}_{22} \mathrm{H}_{38} \mathrm{O}_{4} \mathrm{Si}: 394.2539$.

\section{$\left(2 a R^{*}, 4 S^{*}, 4 a R^{*}, 10 S^{*}\right)-10-($ tert-Butyldimethylsilyl)oxy-1,2,2a,3,4,4a,5,6,9,10-decahydro-4-} (methoxymethoxy)cyclonona[cd]pentalen-7(8H)-one (31)

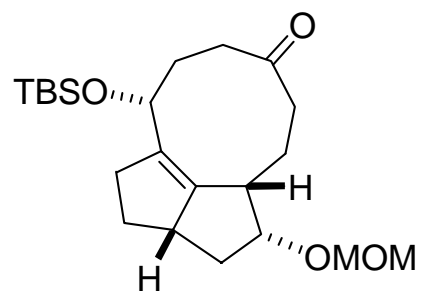

31

18-Crown-6 (29 mg, $0.11 \mathrm{mmol})$ was added to a solution of $\mathbf{3 0}(36 \mathrm{mg}, 0.09 \mathrm{mmol})$ in $\mathrm{THF}$ at 0 ${ }^{\circ} \mathrm{C}$. KH $(6 \mathrm{mg}, 0.1 \mathrm{mmol})$ was then added, and the mixture was allowed to warm to $\mathrm{rt}$. TLC analysis showed the formation of a more polar compound. After $5 \mathrm{~h}$, the reaction was quenched with saturated aqueous $\mathrm{NH}_{4} \mathrm{Cl}$. The aqueous layer was extracted with $\mathrm{CH}_{2} \mathrm{Cl}_{2}(4 \times 5 \mathrm{~mL})$. The combined organic layers were washed with brine and then dried over $\mathrm{MgSO}_{4}$. The solvent was removed in vacuo, and the residue was purified by flash chromatography (10\% EtOAc/hexanes) to afford 31 (24 mg, 67\%) as colorless crystals. mp: 60-61 ${ }^{\circ} \mathrm{C}$. IR: 2932 (s), 2857 (s), 1702 (s) cm ${ }^{-1} ;{ }^{1} \mathrm{H}$ NMR: $\delta 4.69$ (1H, d, J = 6.4 Hz), 4.65 $(1 \mathrm{H}, \mathrm{d}, J=6.4 \mathrm{~Hz}), 4.44(1 \mathrm{H}, \mathrm{dd}, J=11.1,2.5 \mathrm{~Hz}), 4.29(1 \mathrm{H}, \mathrm{ddd}, J=11.3,9.8,6.4 \mathrm{~Hz}), 3.38(3 \mathrm{H}, \mathrm{s})$, $2.89(1 \mathrm{H}, \mathrm{m}), 2.73(1 \mathrm{H}, \mathrm{ddd}, J=16.8,13.0,4.0 \mathrm{~Hz}), 2.63(1 \mathrm{H}, \mathrm{m}), 2.50-2.32(4 \mathrm{H}, \mathrm{m}), 2.29-2.13(4 \mathrm{H}$, m), $2.03(1 \mathrm{H}, \mathrm{dt}, J=13.0,4.0 \mathrm{~Hz}), 1.89(1 \mathrm{H}, \mathrm{m}), 1.81(1 \mathrm{H}, \mathrm{m}), 1.41(1 \mathrm{H}, \mathrm{p}, J=10.4 \mathrm{~Hz}), 1.28(1 \mathrm{H}, \mathrm{q}, J$ $=11.6 \mathrm{~Hz}), 0.87(9 \mathrm{H}, \mathrm{s}), 0.02(3 \mathrm{H}, \mathrm{s}),-0.01(3 \mathrm{H}, \mathrm{s}) ;{ }^{13} \mathrm{C} \mathrm{NMR}: \delta 214.4(0), 145.5(0), 136.4(0), 96.1$ (2), 82.6 (1), 70.4 (1), 55.4 (3), 48.1 (1), 44.8 (2), 38.3 (2), 37.1 (1), 35.7 (2), 34.8 (2), 34.4 (2), 29.9 (2), 25.8 (3C, 3), 25.1 (2), $18.4(0),-4.9$ (3), -5.1 (3); HRMS: $m / z 394.2518\left(\mathrm{M}^{+}\right)$, required for $\mathrm{C}_{22} \mathrm{H}_{38} \mathrm{O}_{4} \mathrm{Si}$ : 394.2539. 
NMR spectra of 1-(trimethylsilyl)hept-6-en-1-yn-3-one (9)

${ }^{1} \mathrm{H} \mathrm{NMR}\left(\mathrm{CDCl}_{3}, 500 \mathrm{MHz}\right)$
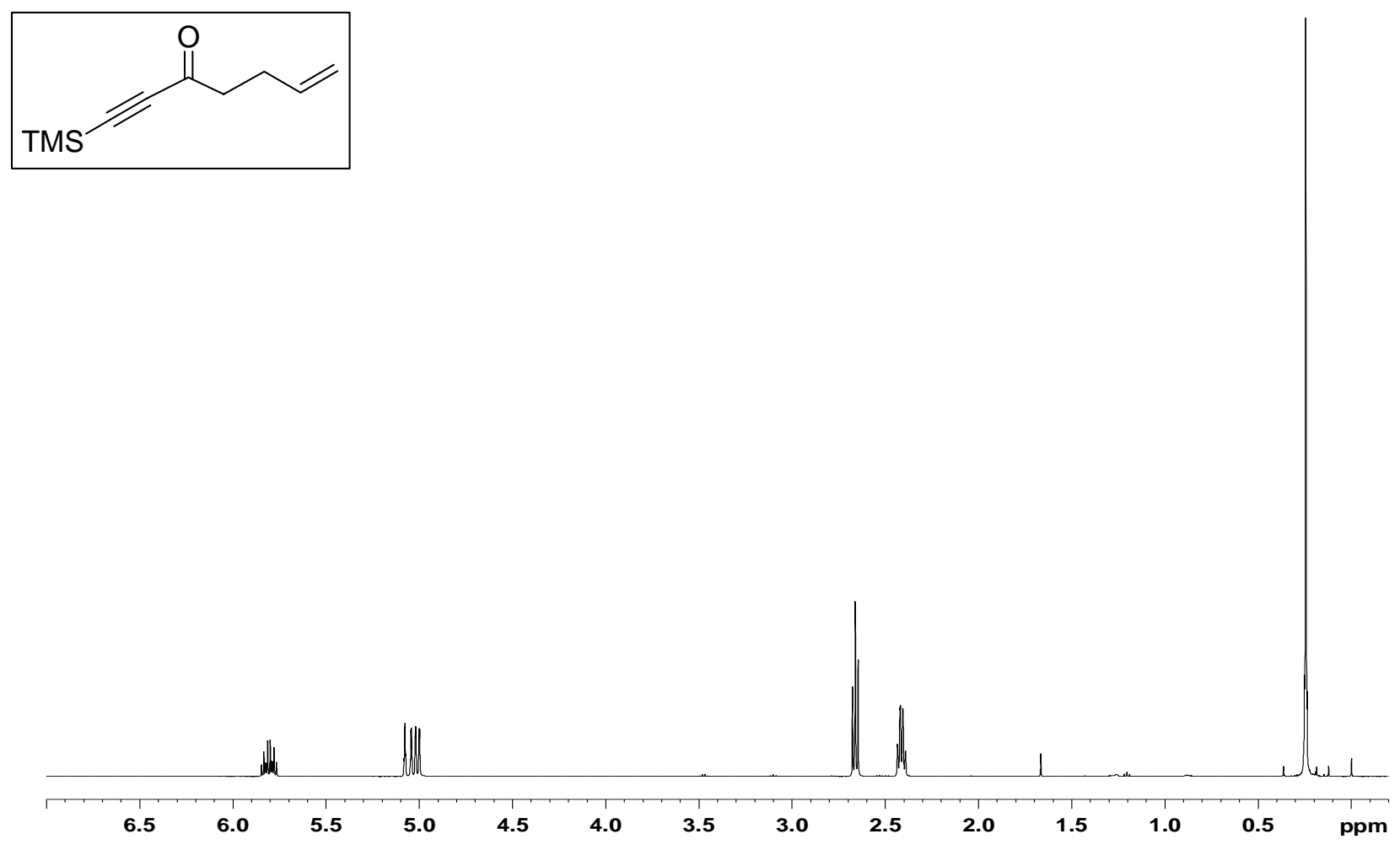

${ }^{13} \mathrm{C} \mathrm{NMR}\left(\mathrm{CDCl}_{3}, 125 \mathrm{MHz}\right)$

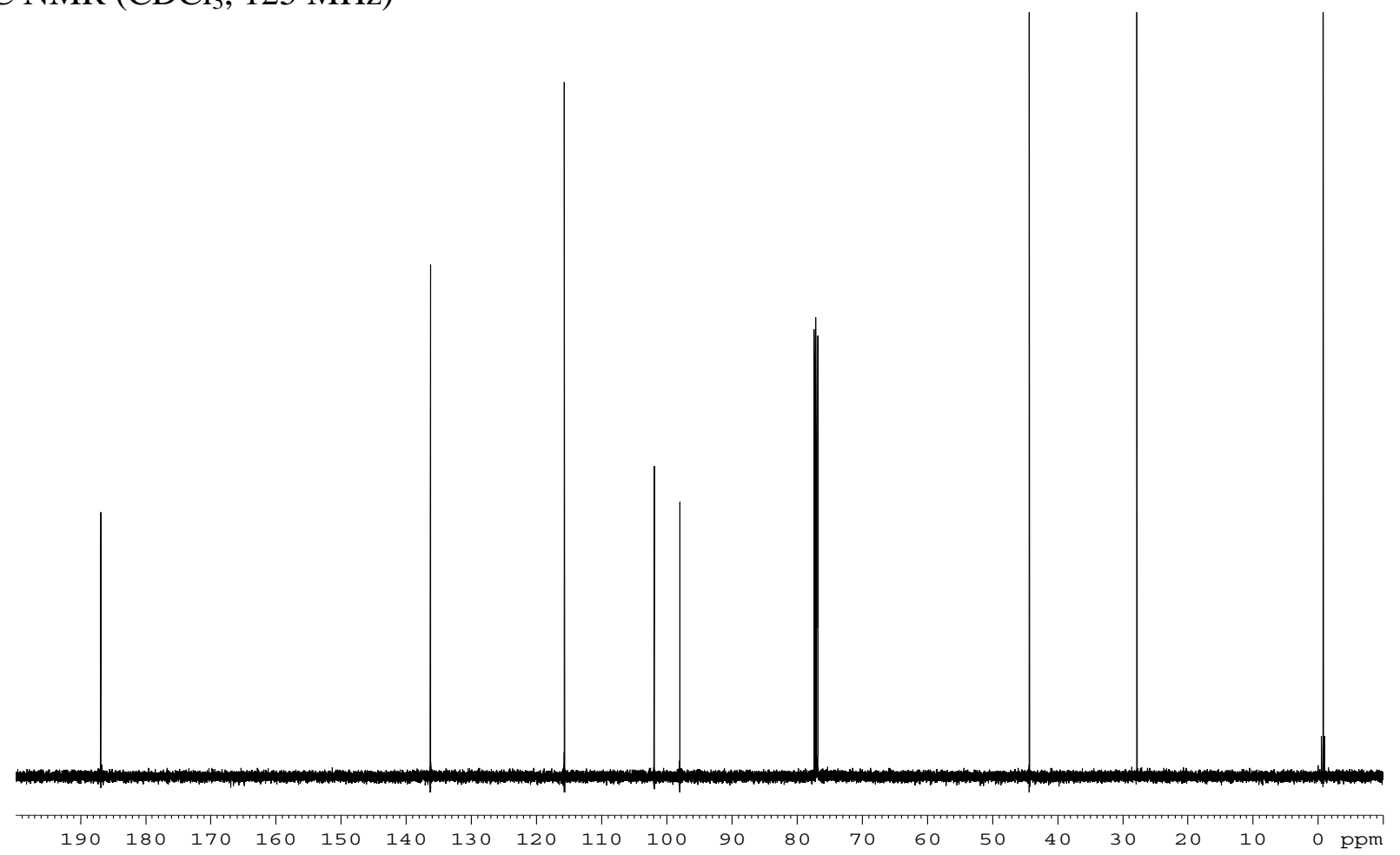


NMR spectra of 2-(but-3-enyl)-2-((trimethylsilyl)ethynyl)cyclopentane-1,3-dione (8)

${ }^{1} \mathrm{H} \mathrm{NMR}\left(\mathrm{CDCl}_{3}, 500 \mathrm{MHz}\right)$
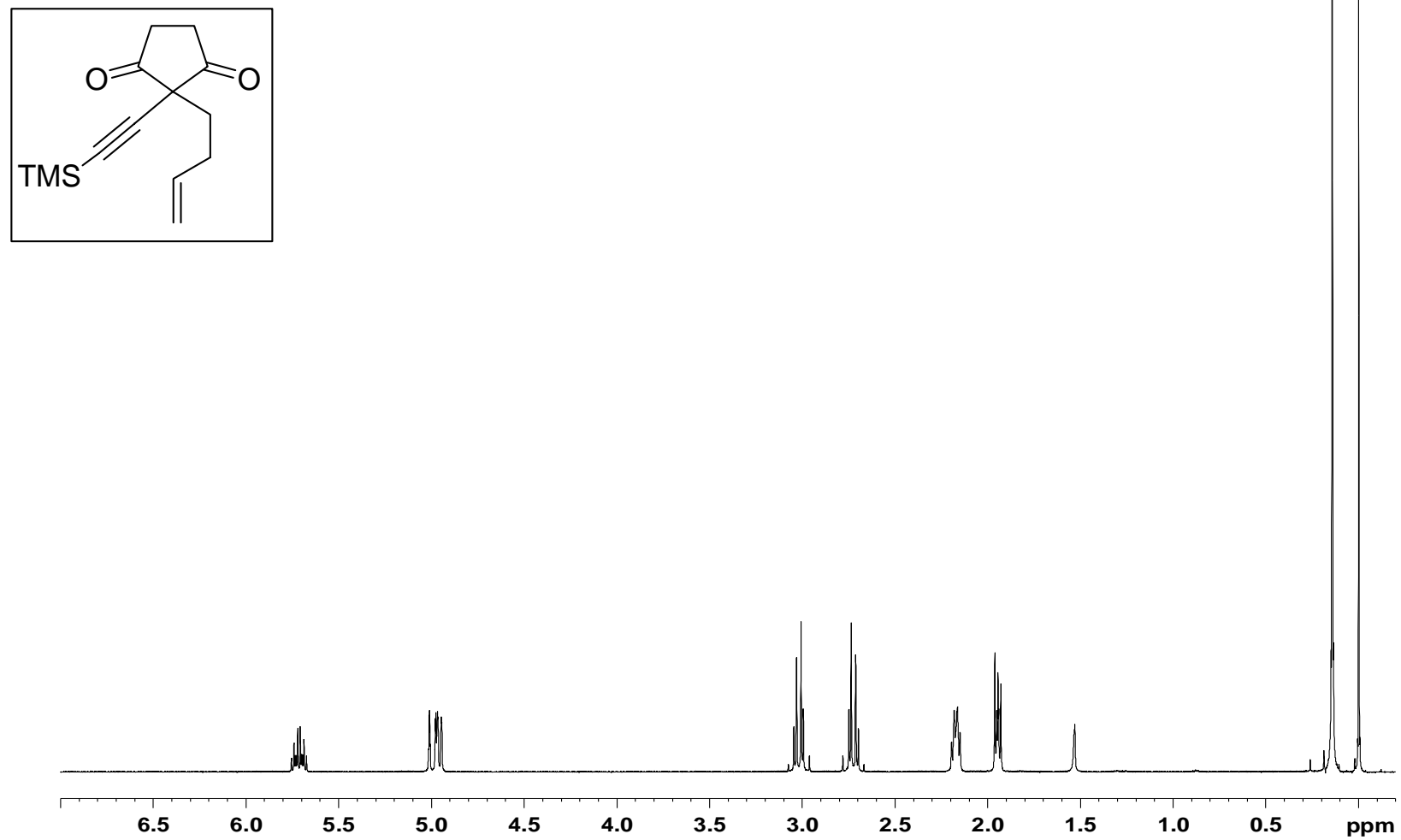

${ }^{13} \mathrm{C} \mathrm{NMR}\left(\mathrm{CDCl}_{3}, 125 \mathrm{MHz}\right)$

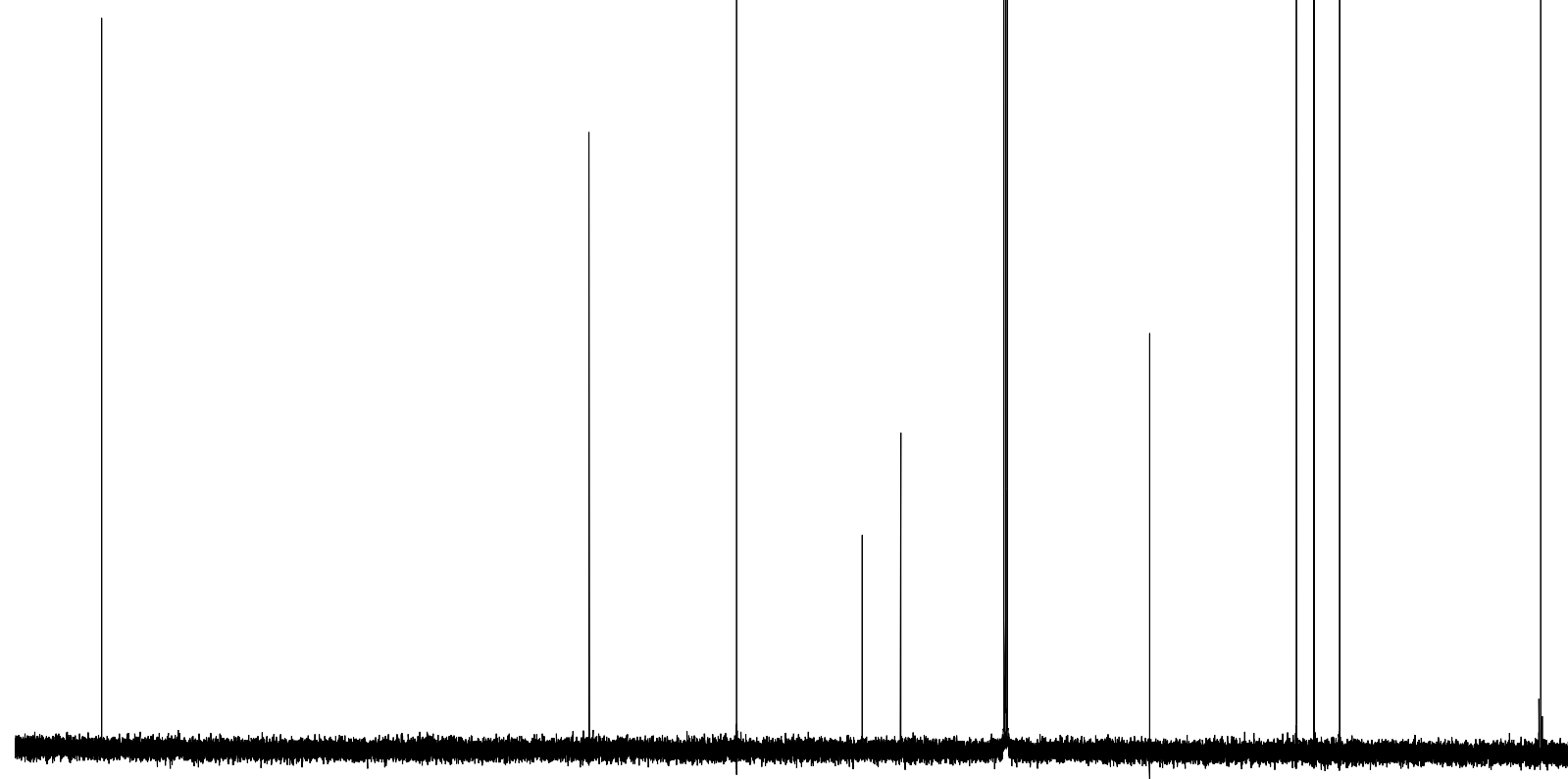

$\begin{array}{llllllllllllllllllllll}210 & 200 & 190 & 180 & 170 & 160 & 150 & 140 & 130 & 120 & 110 & 100 & 90 & 80 & 70 & 60 & 50 & 40 & 30 & 20 & 10 & \mathrm{ppm}\end{array}$ 
NMR spectra of $\left(2 R^{*}, 3 R^{*}\right)-2$-(but-3-enyl)-3-hydroxy-2-((trimethylsilyl)ethynyl)cyclopentanone (12)

${ }^{1} \mathrm{H} \mathrm{NMR}\left(\mathrm{CDCl}_{3}, 500 \mathrm{MHz}\right)$
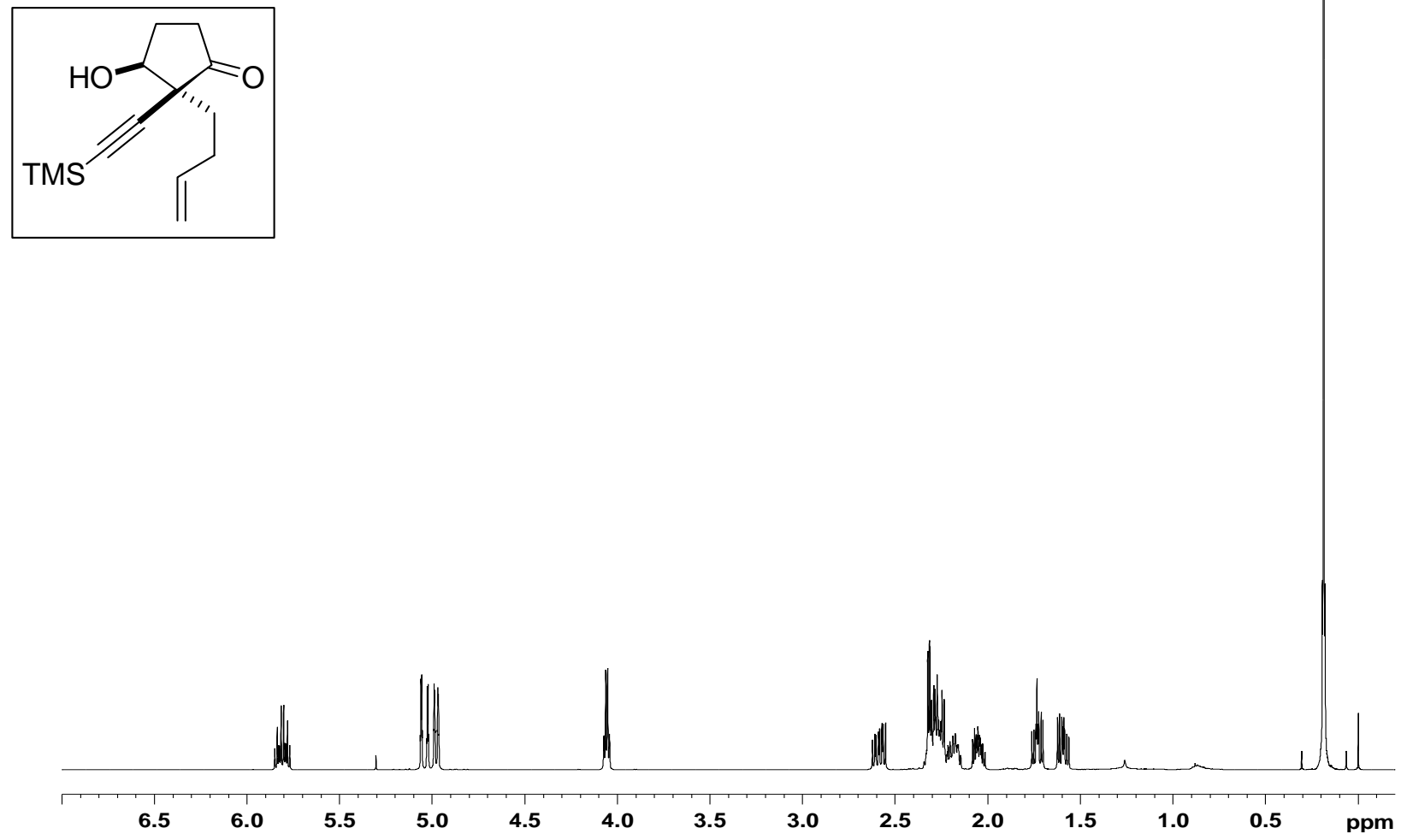

${ }^{13} \mathrm{C} \mathrm{NMR}\left(\mathrm{CDCl}_{3}, 125 \mathrm{MHz}\right)$

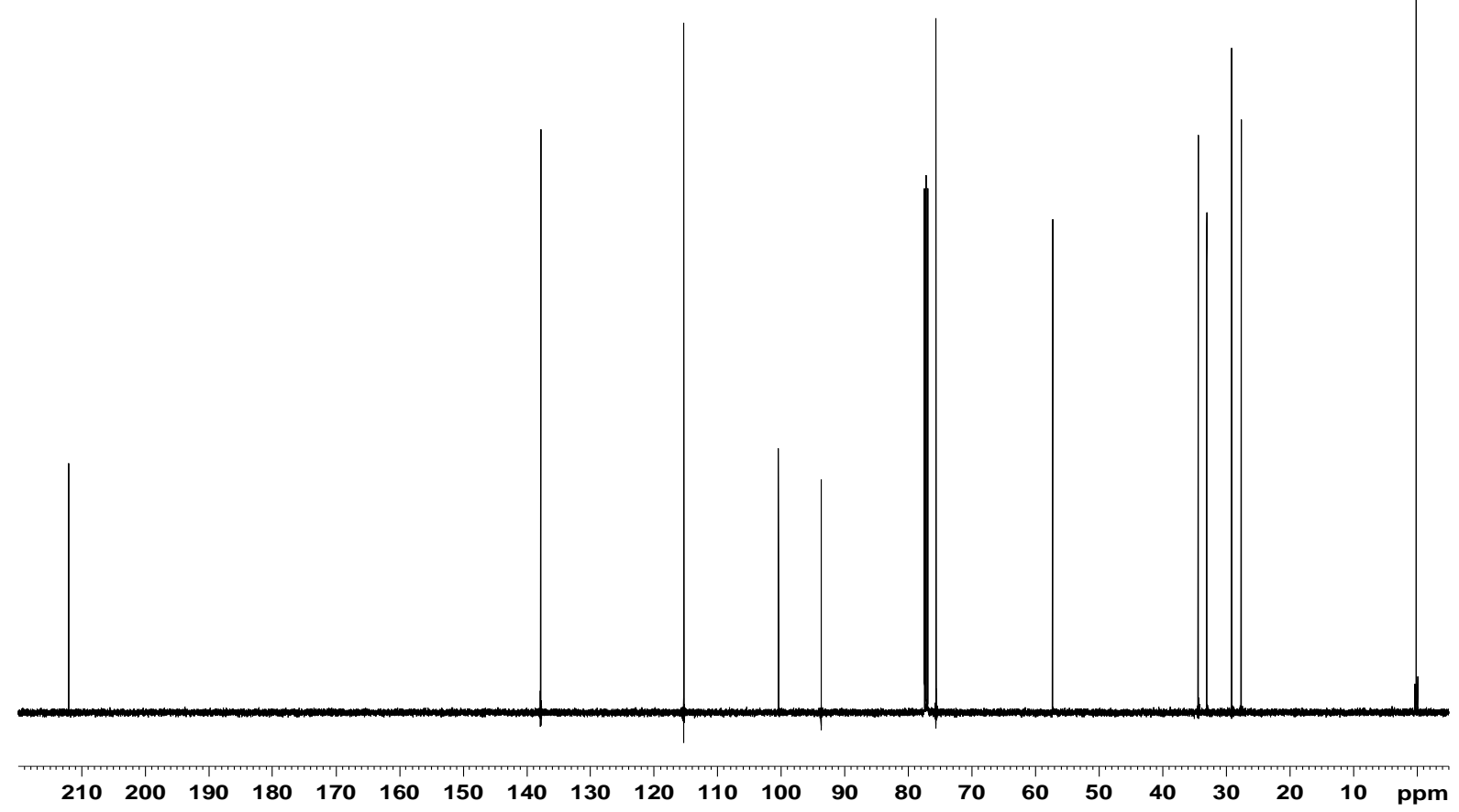


NMR spectra of $\left(2 R^{*}, 3 S^{*}\right)-2-($ But-3-enyl)-3-hydroxy-2-((trimethylsilyl)ethynyl)cyclopentanone (13)

${ }^{1} \mathrm{H} \mathrm{NMR}\left(\mathrm{CDCl}_{3}, 500 \mathrm{MHz}\right)$
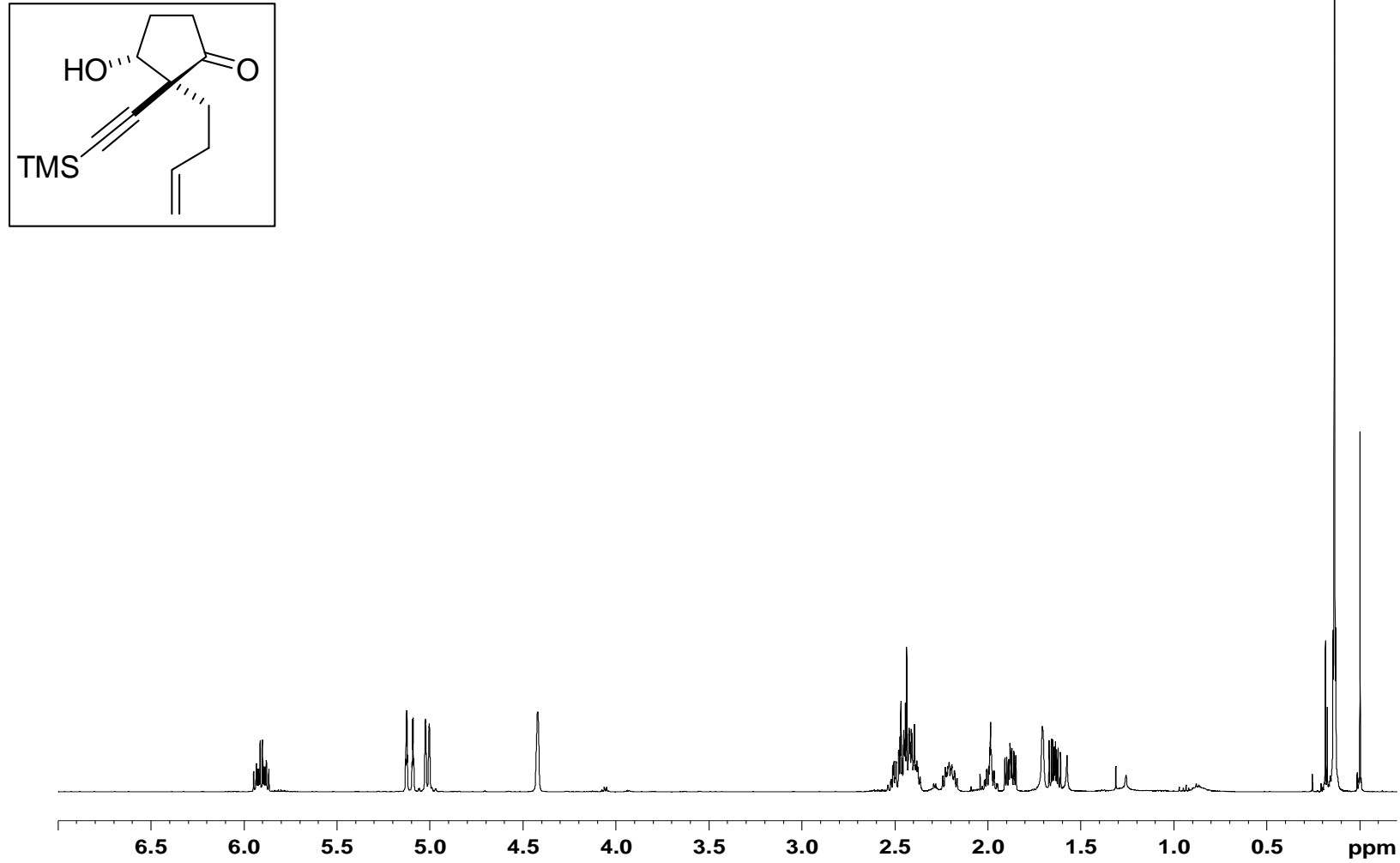

${ }^{13} \mathrm{C} \mathrm{NMR}\left(\mathrm{CDCl}_{3}, 125 \mathrm{MHz}\right)$

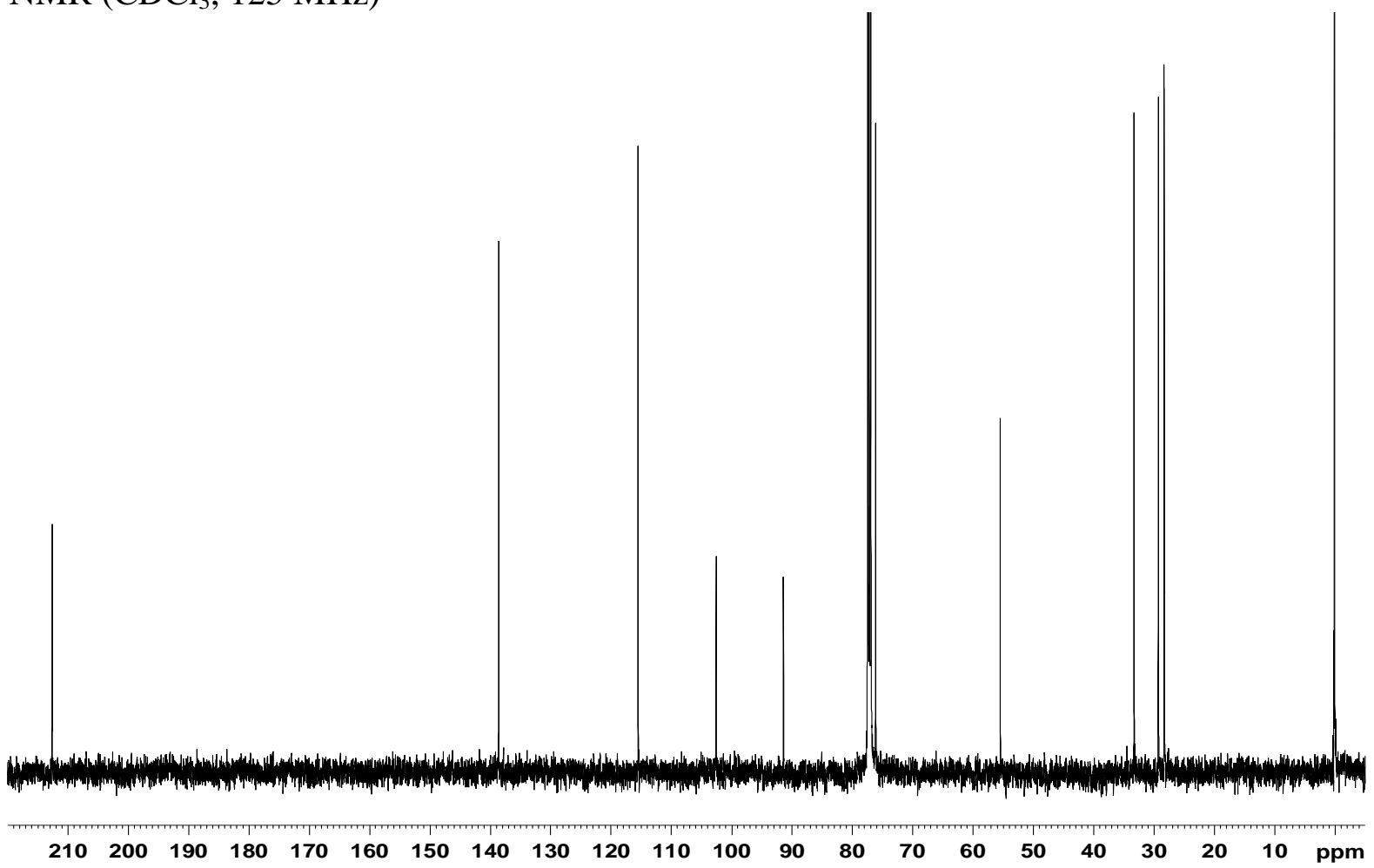


NMR spectra of $\left(2 R^{*}, 3 R^{*}\right)-2$-(but-3-enyl)-3-(tert-butyldimethylsilyl)oxy-2-((trimethylsilyl)ethynyl)cyclopentanone

${ }^{1} \mathrm{H} \mathrm{NMR}\left(\mathrm{CDCl}_{3}, 500 \mathrm{MHz}\right)$
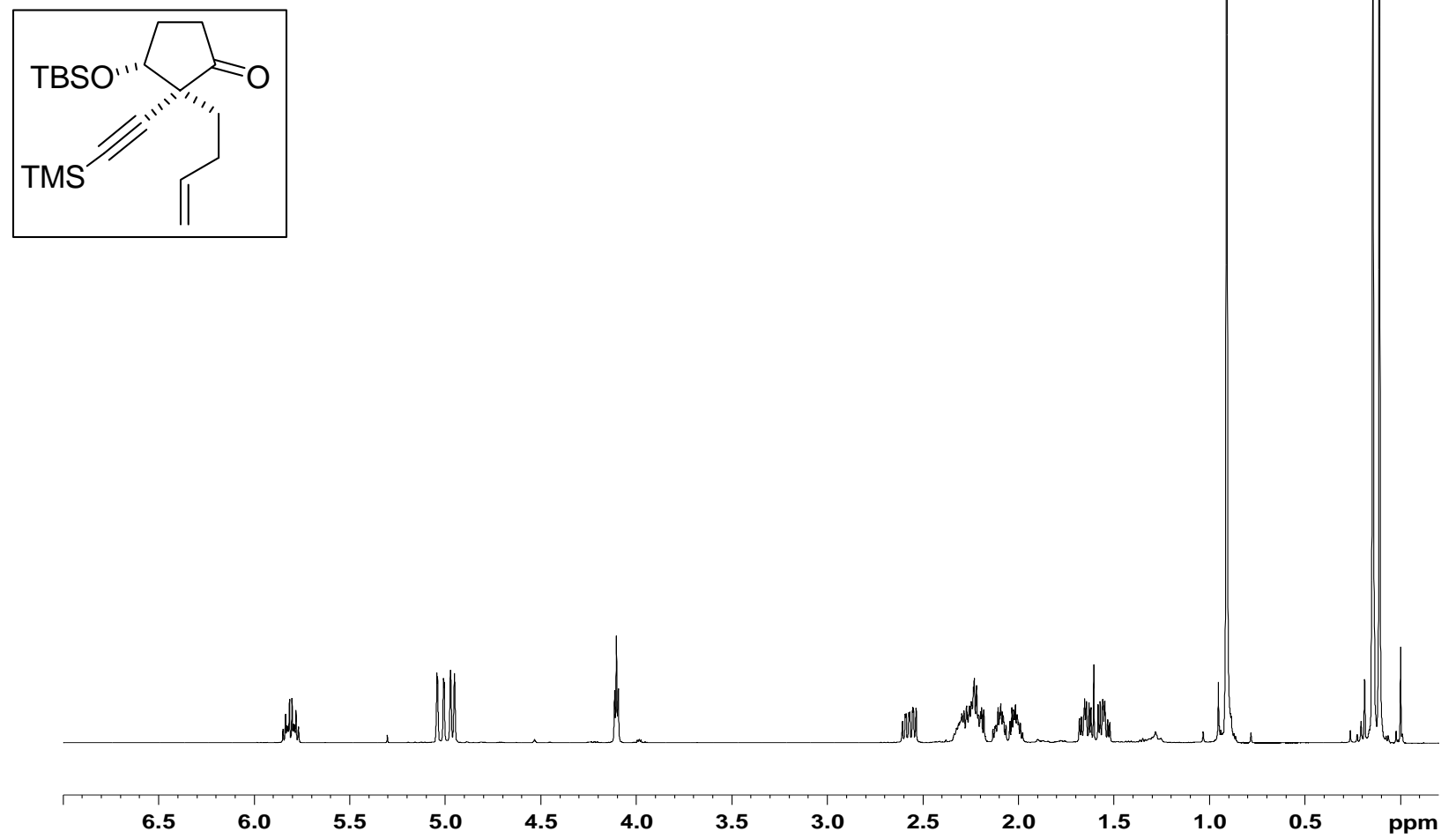

${ }^{13} \mathrm{C} \mathrm{NMR}\left(\mathrm{CDCl}_{3}, 125 \mathrm{MHz}\right)$

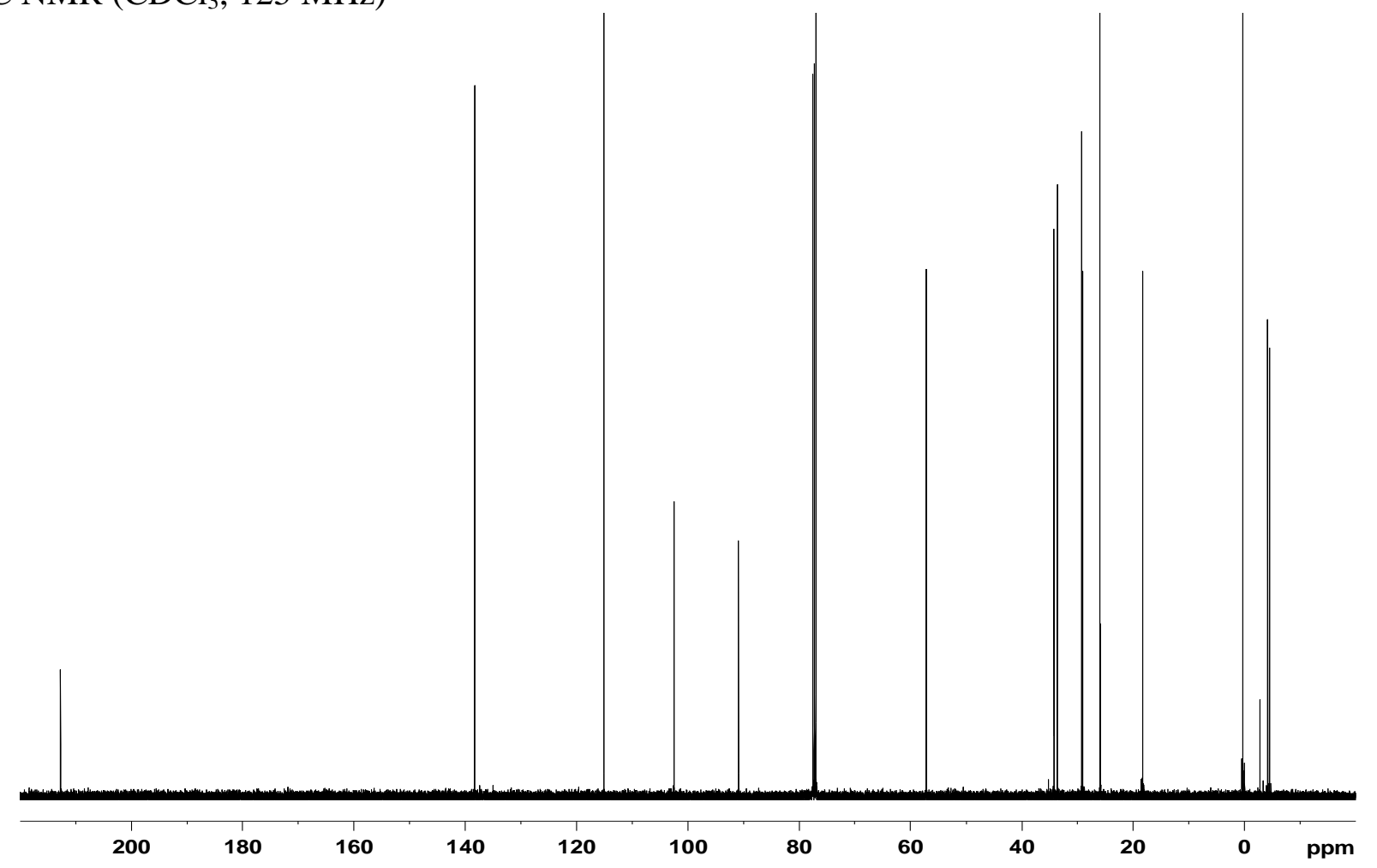


NMR spectra of $\left(1 R^{*}, 2 R^{*}, 3 R^{*}\right)-2$-(but-3-enyl)-3-(tert-butyldimethylsilyl)oxy-2-((trimethylsilyl)ethynyl)-1-vinylcyclopentanol

${ }^{1} \mathrm{H} \mathrm{NMR}\left(\mathrm{CDCl}_{3}, 500 \mathrm{MHz}\right)$
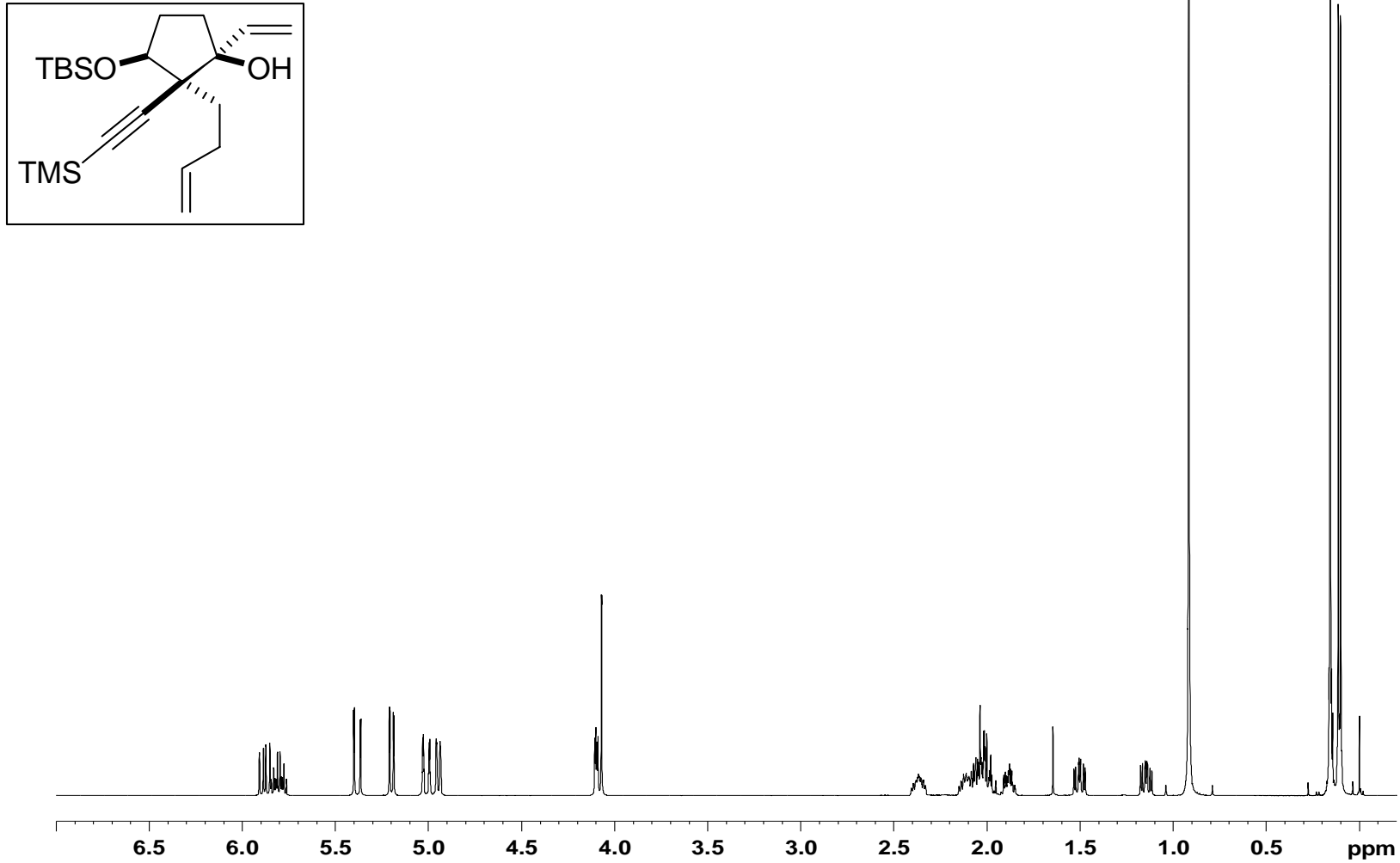

${ }^{13} \mathrm{C} \mathrm{NMR}\left(\mathrm{CDCl}_{3}, 125 \mathrm{MHz}\right)$

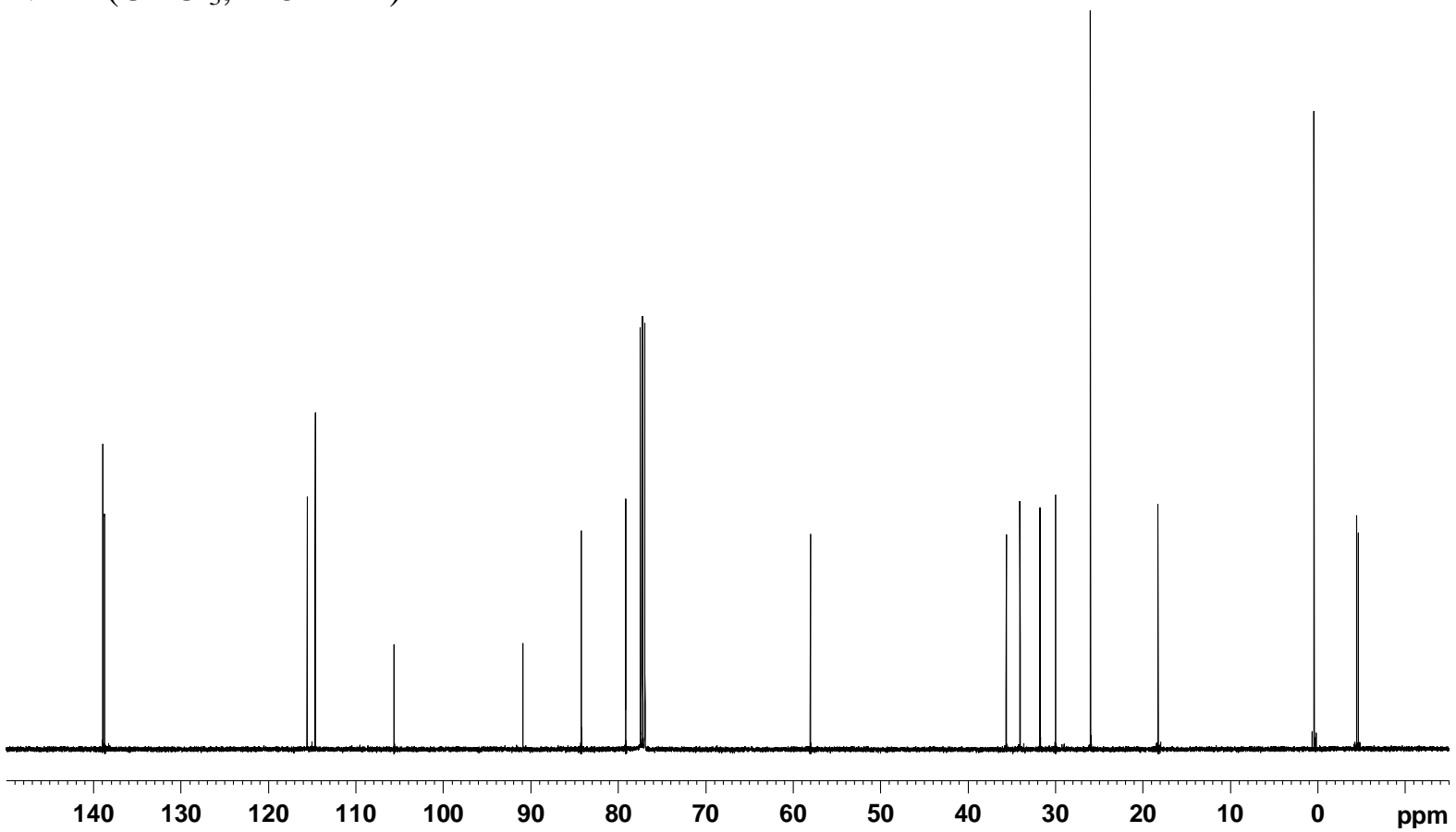


NMR spectra of $\left(1 R^{*}, 2 R^{*}, 3 R^{*}\right)$-2-(but-3-enyl)-3-(tert-butyldimethylsilyl)oxy-2-ethynyl-1-vinylcyclopentanol (14)

${ }^{1} \mathrm{H} \mathrm{NMR}\left(\mathrm{CDCl}_{3}, 500 \mathrm{MHz}\right)$
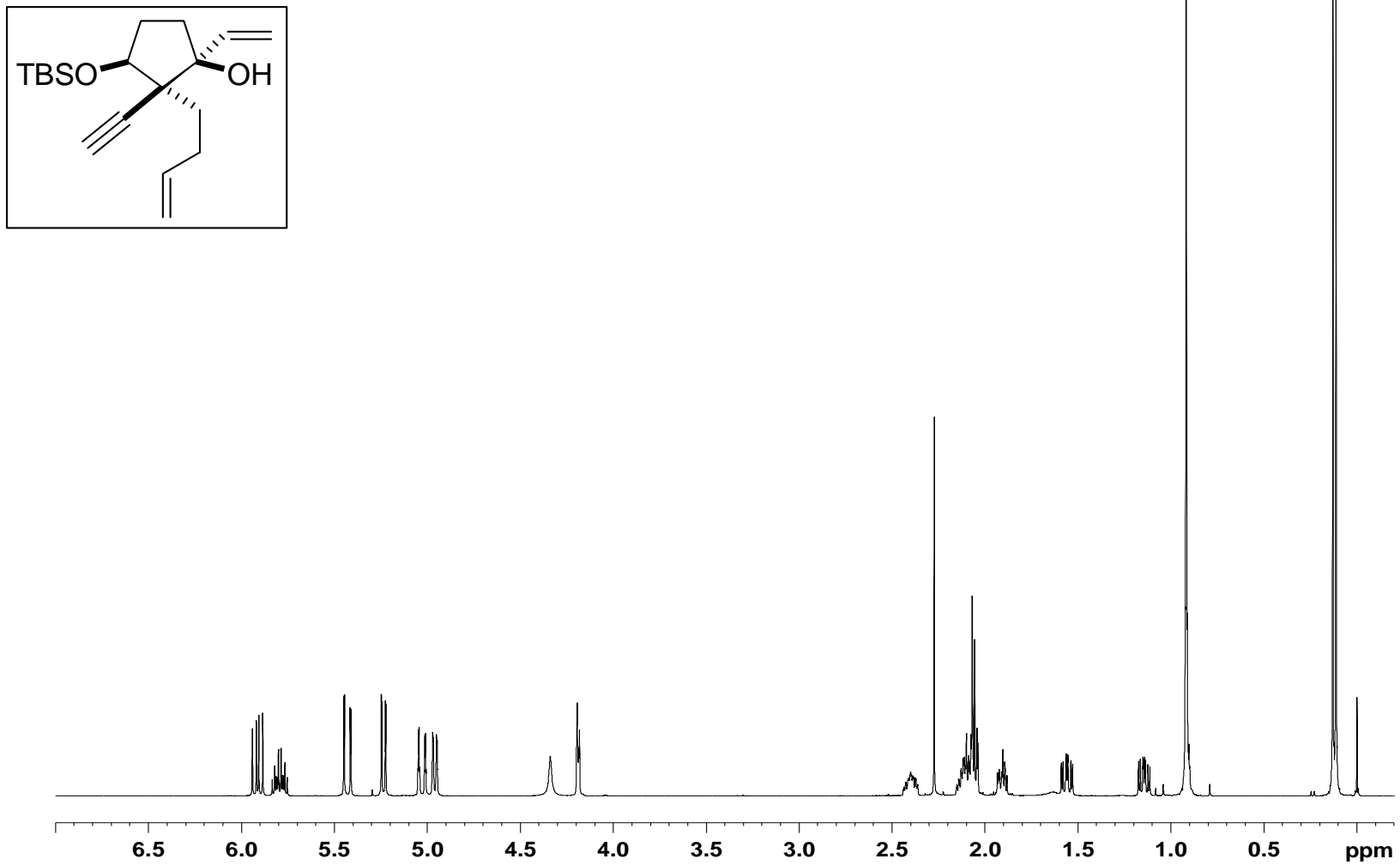

${ }^{13} \mathrm{C} \mathrm{NMR}\left(\mathrm{CDCl}_{3}, 125 \mathrm{MHz}\right)$

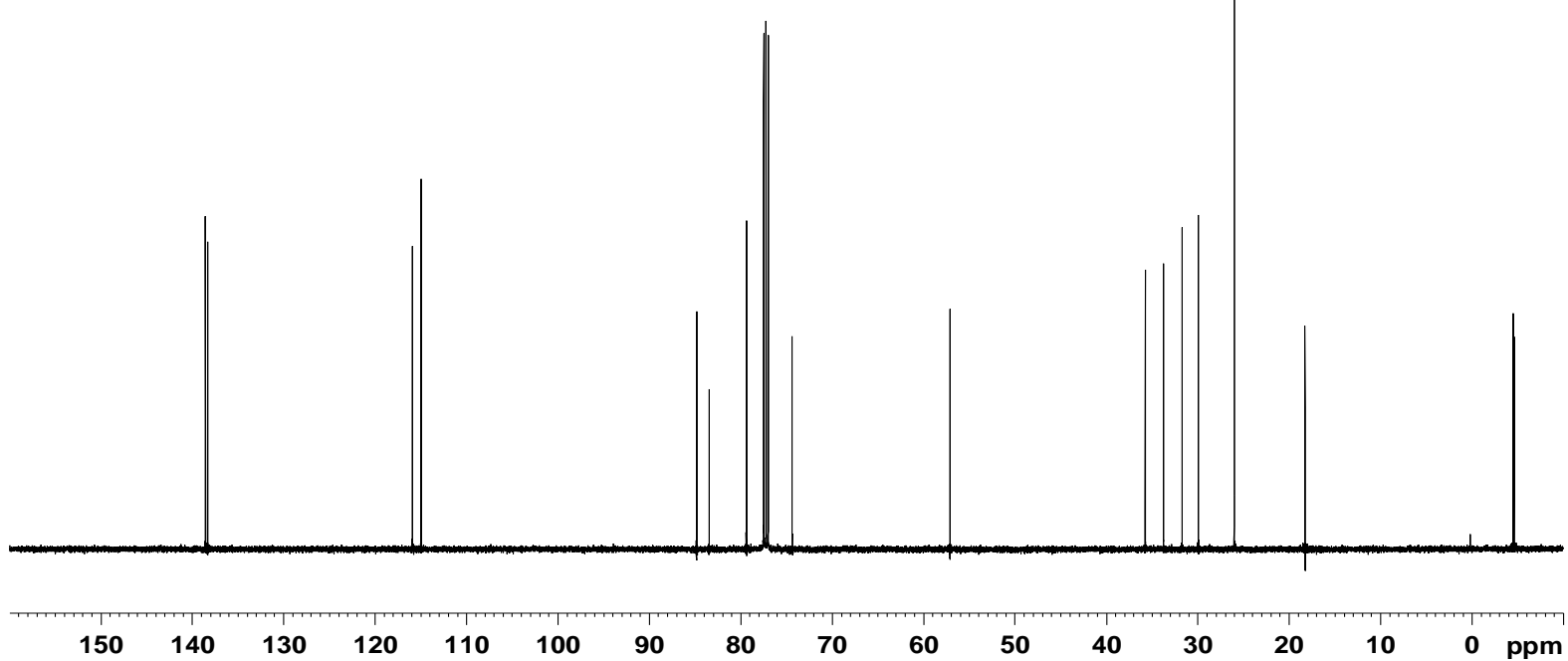


NMR spectra of $\left(1 R^{*}, 2 R^{*}, 3 a^{\prime} R^{*}, 5 R^{*}\right)$-5-(tert-butyldimethylsilyl)oxy-2',3',3 $a^{\prime}, 4^{\prime}$-tetrahydro-2hydroxy-2-vinyl-5' $H$-spiro[cyclopentane-1,1'-pentalen]-5'-one (15)

${ }^{1} \mathrm{H} \mathrm{NMR}\left(\mathrm{CDCl}_{3}, 500 \mathrm{MHz}\right)$
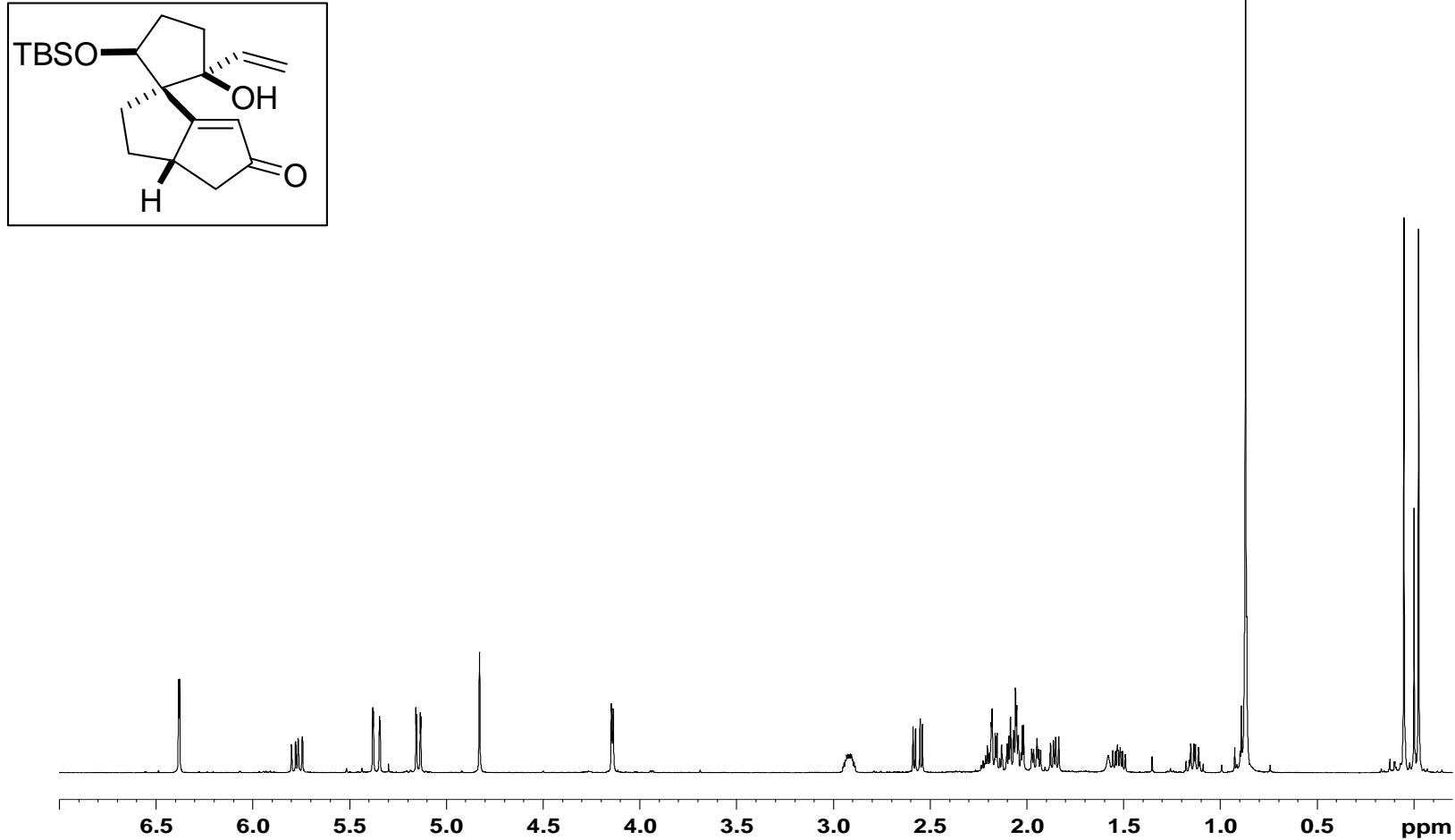

${ }^{13} \mathrm{C} \mathrm{NMR}\left(\mathrm{CDCl}_{3}, 125 \mathrm{MHz}\right)$

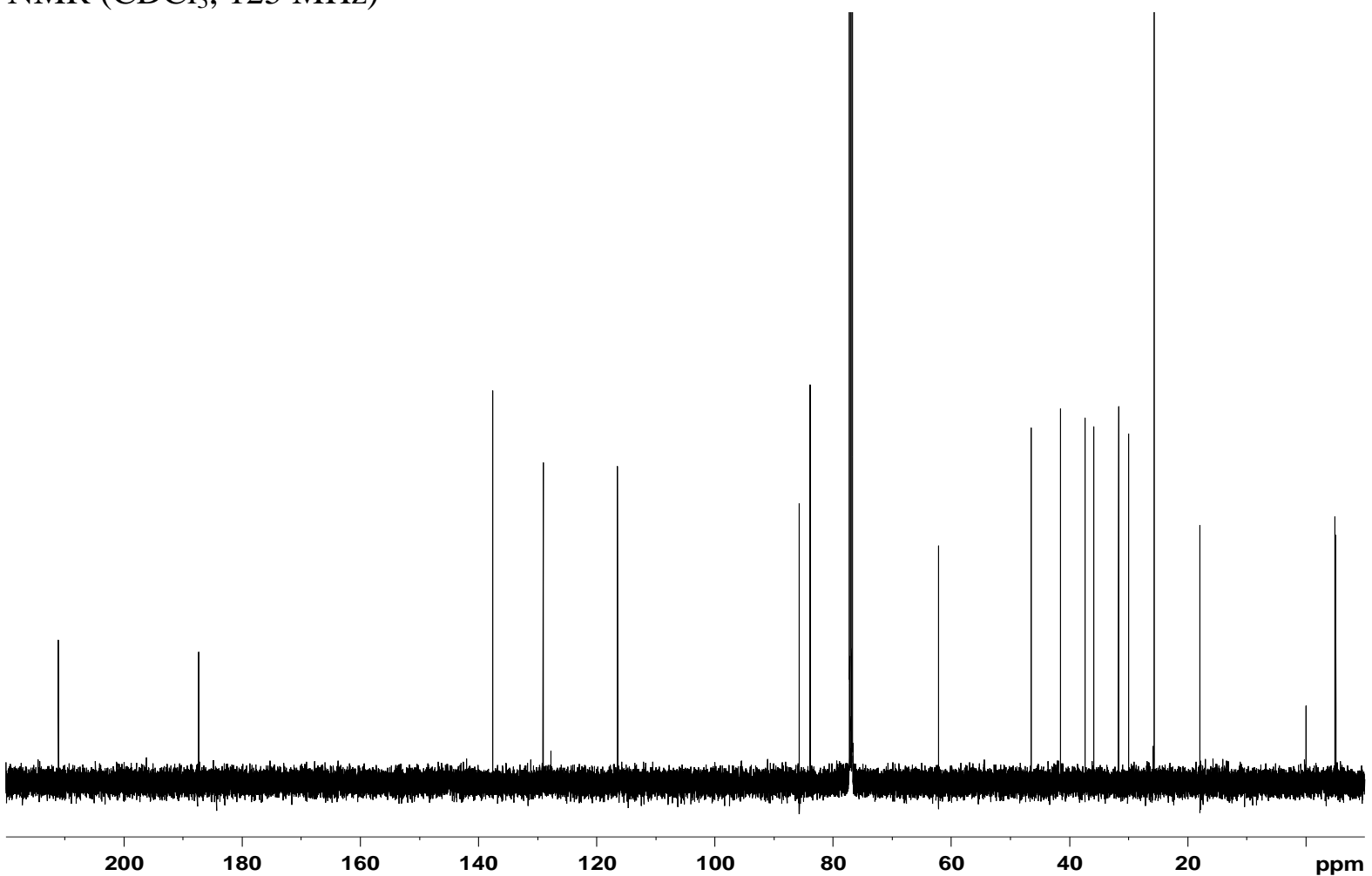


NMR spectra of $\left(1 R^{*}, 2 R^{*}, 3 a^{\prime} S^{*}, 5 R^{*}\right)$-5-(tert-butyldimethylsilyl)oxy-2',3',3 $a^{\prime}, 4^{\prime}$-tetrahydro-2hydroxy-2-vinyl-5' $H$-spiro[cyclopentane-1,1'-pentalen]-5'-one (16)

${ }^{1} \mathrm{H} \mathrm{NMR}\left(\mathrm{CDCl}_{3}, 500 \mathrm{MHz}\right)$
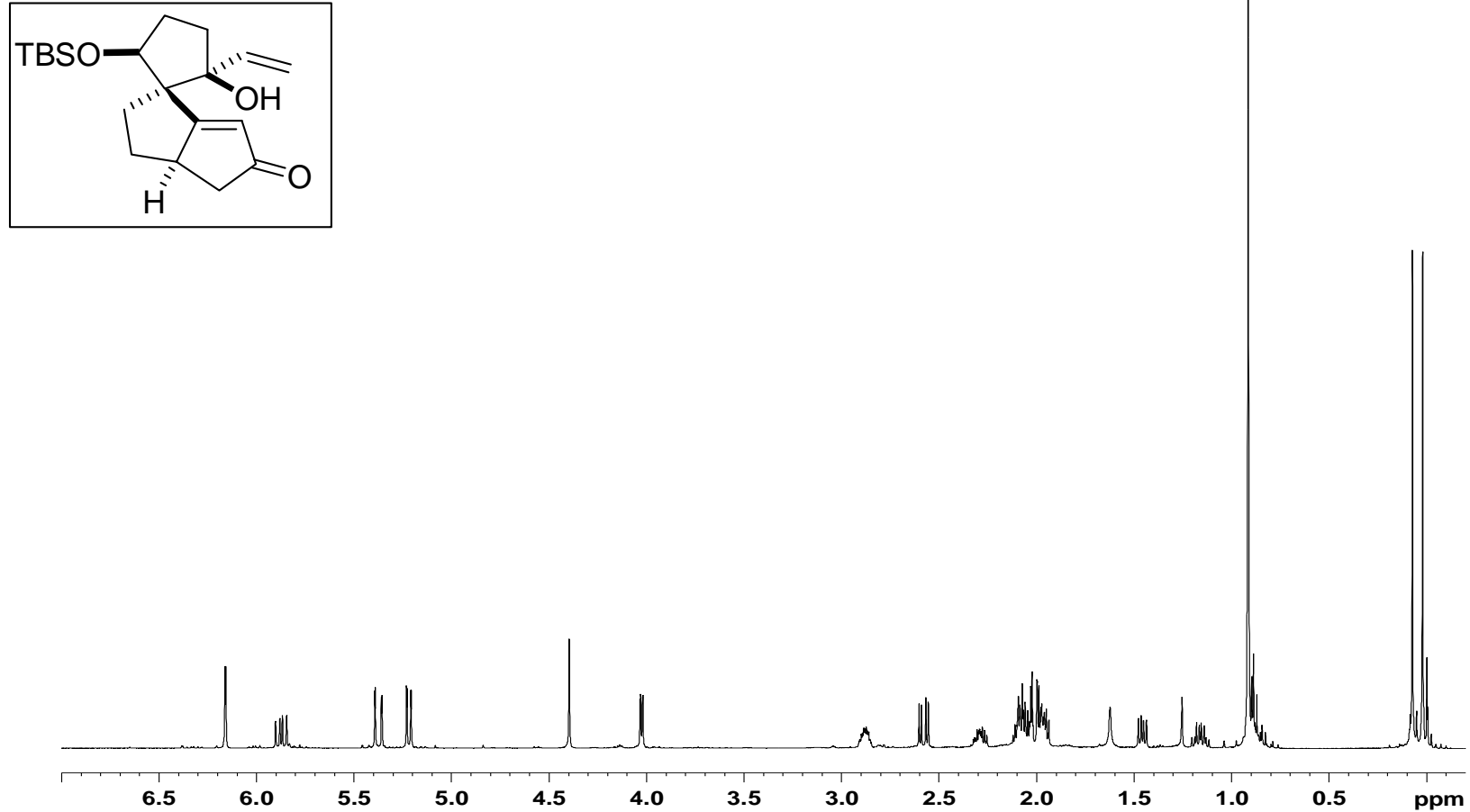

${ }^{13} \mathrm{C} \mathrm{NMR}\left(\mathrm{CDCl}_{3}, 125 \mathrm{MHz}\right)$

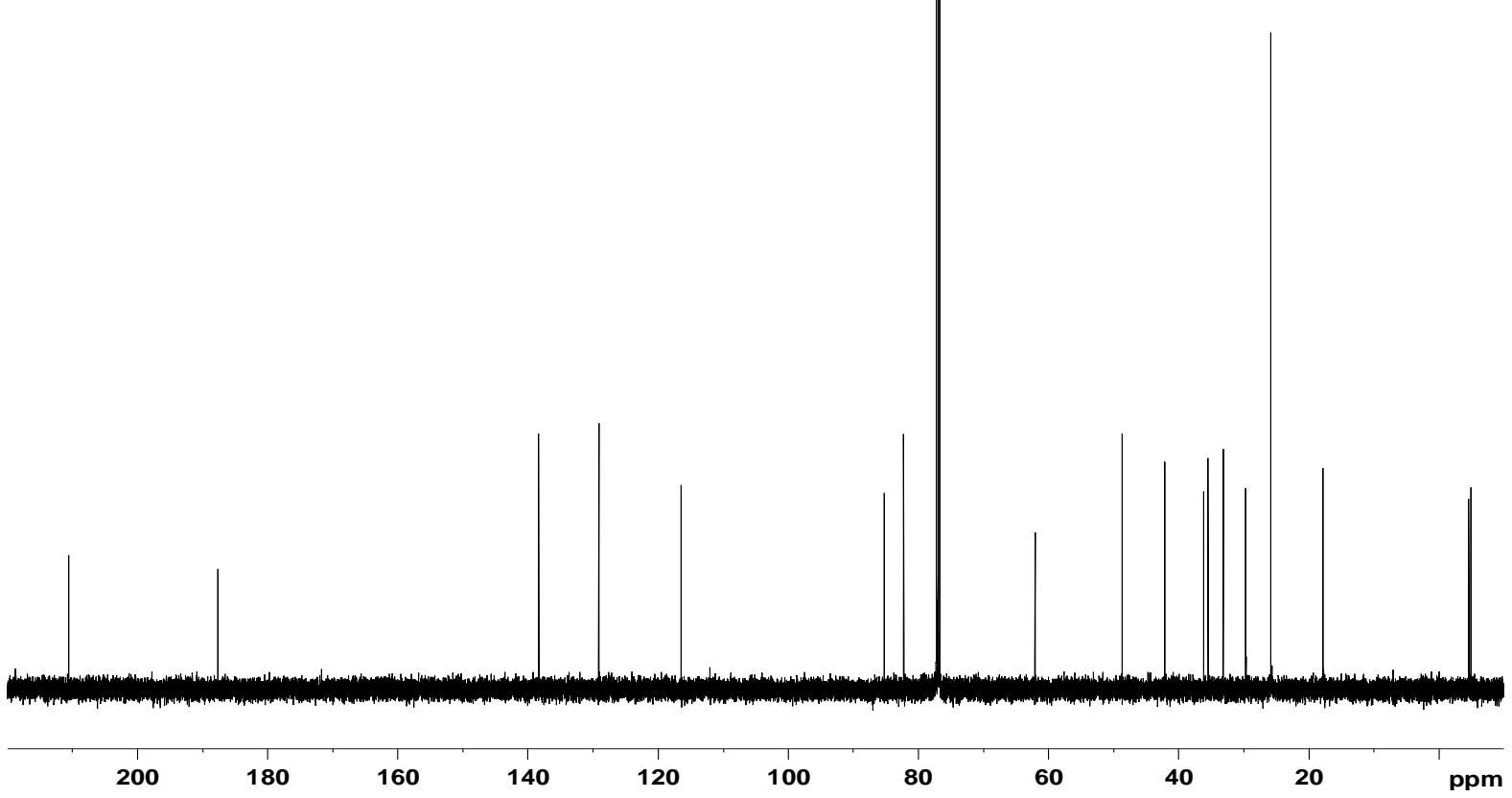


NMR spectra of $\left(4 a R^{*}, 7 R^{*}, 7 a R^{*}, 9 a R^{*}\right)-7-($ tert-butyldimethylsilyl)oxy-3,4,4a,5,6,7,9,9a-octahydro4a-hydroxy-1H-cyclopent $[\mathrm{kl}]$-as-indacen-2(8H)-one (17)

${ }^{1} \mathrm{H} \mathrm{NMR}\left(\mathrm{CDCl}_{3}, 500 \mathrm{MHz}\right)$
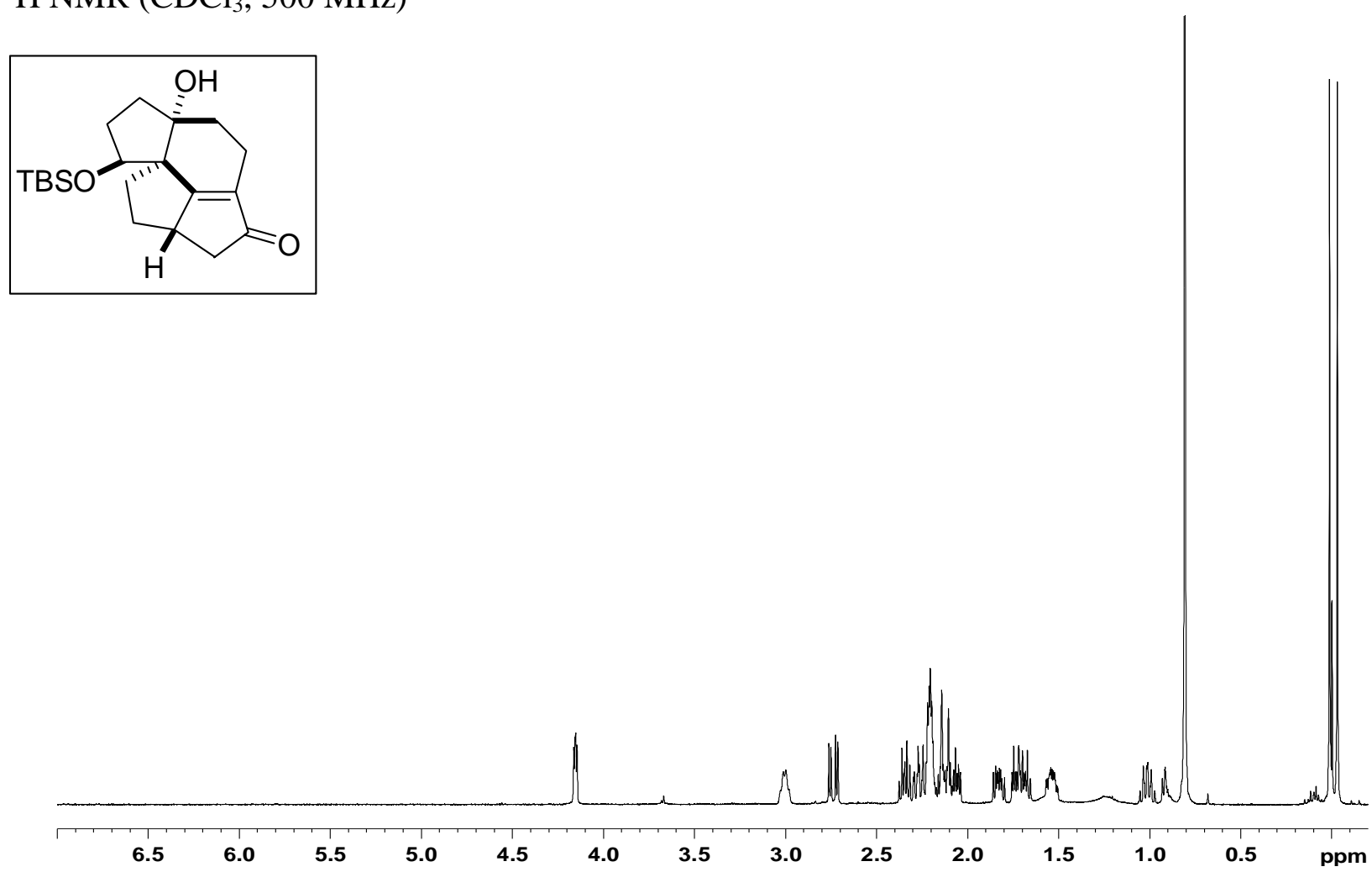

${ }^{13} \mathrm{C} \mathrm{NMR}\left(\mathrm{CDCl}_{3}, 125 \mathrm{MHz}\right)$

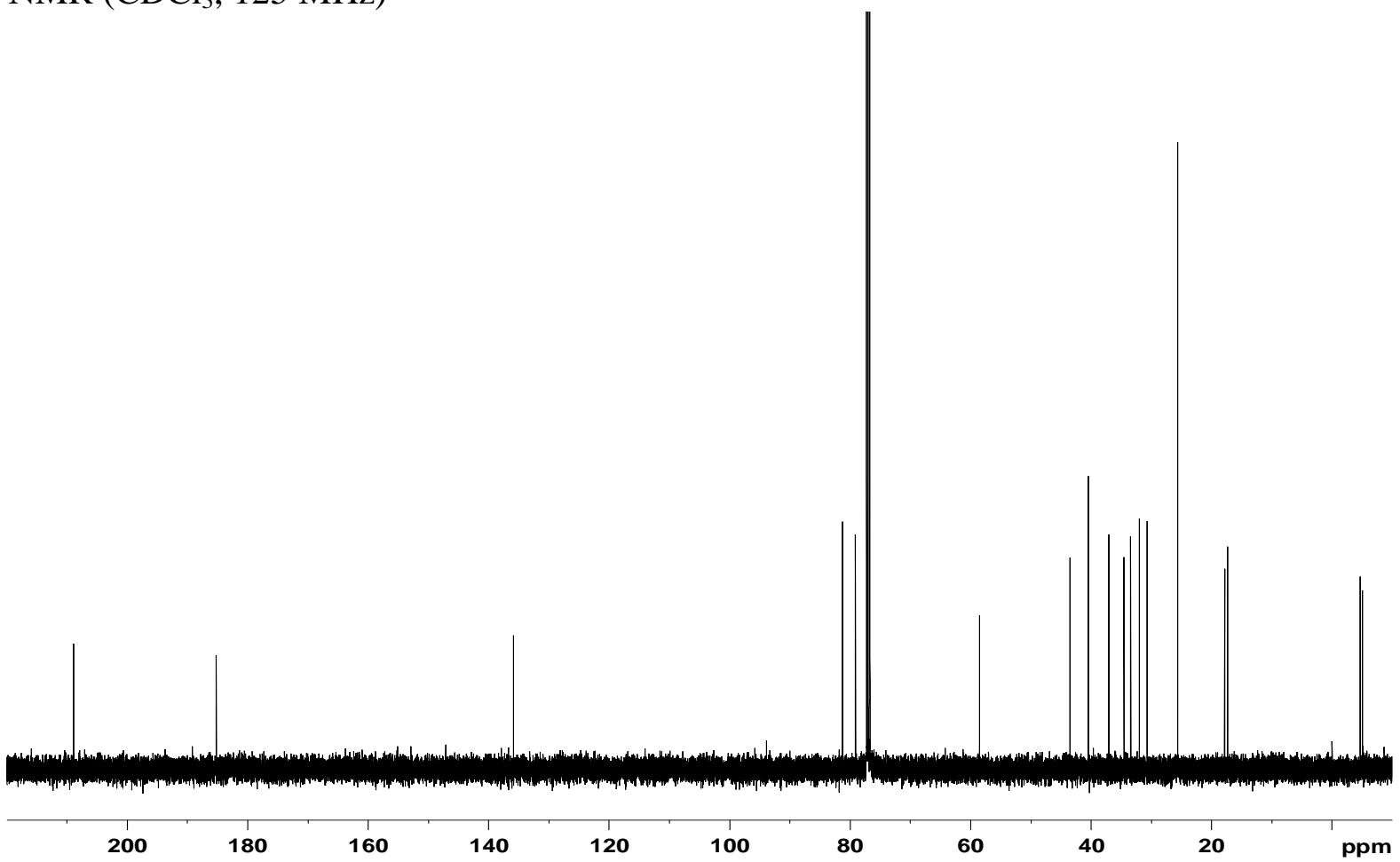


NMR spectra of $\left(2 a R^{*}, 4 a S^{*}, 5 R^{*}, 6 a R^{*}, 9 a S^{*}, 9 b R^{*}\right)-5$-(tert-butyldimethylsilyl)oxy-2,2a,3,4,4a,5,6, 6a,9,9a-decahydro-1H-pentaleno[1,6-dc]indene-1,7(8H)-dione (18)

${ }^{1} \mathrm{H}$ NMR $\left(\mathrm{C}_{6} \mathrm{D}_{6}, 500 \mathrm{MHz}\right)$
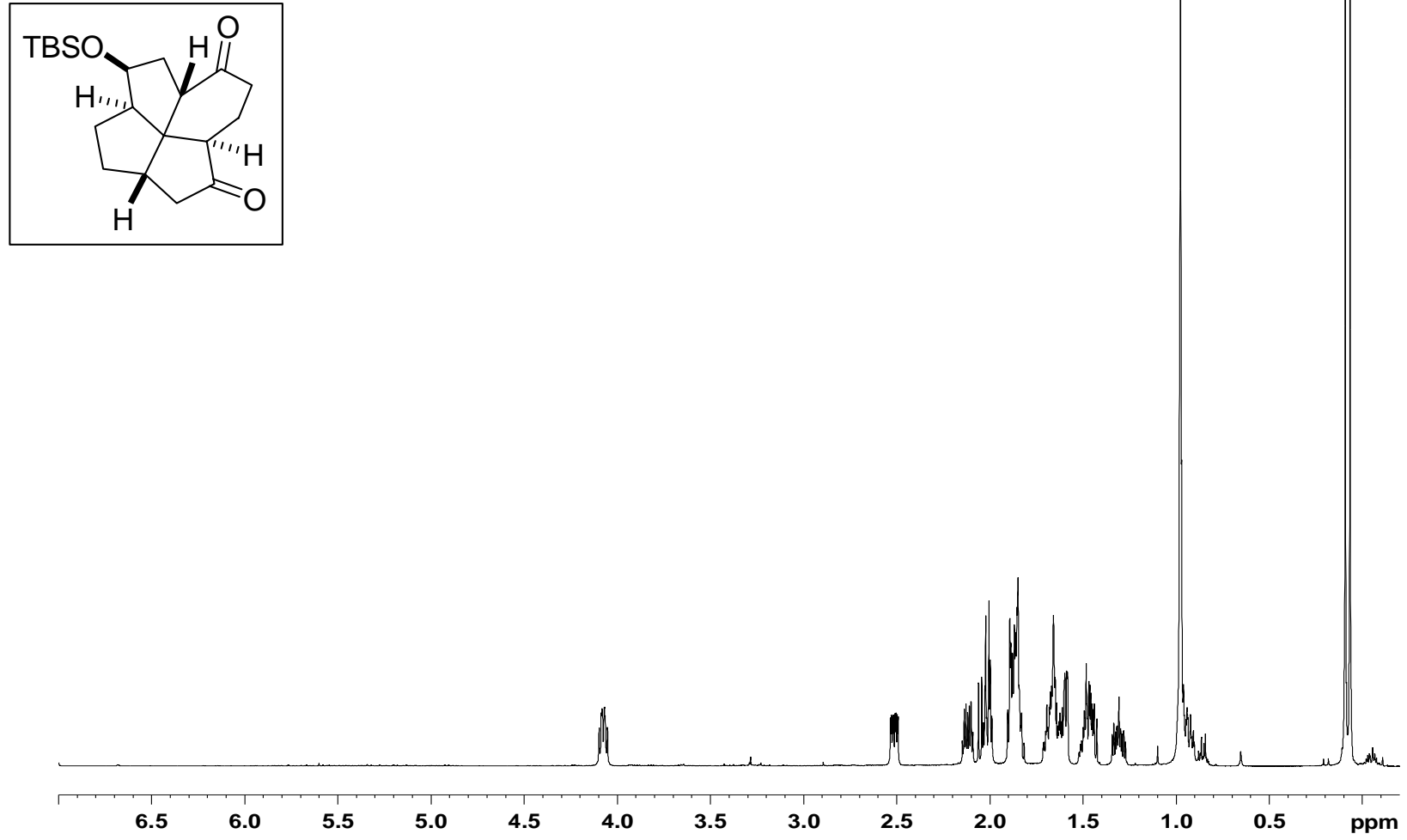

${ }^{13} \mathrm{C}$ NMR $\left(\mathrm{C}_{6} \mathrm{D}_{6}, 125 \mathrm{MHz}\right)$

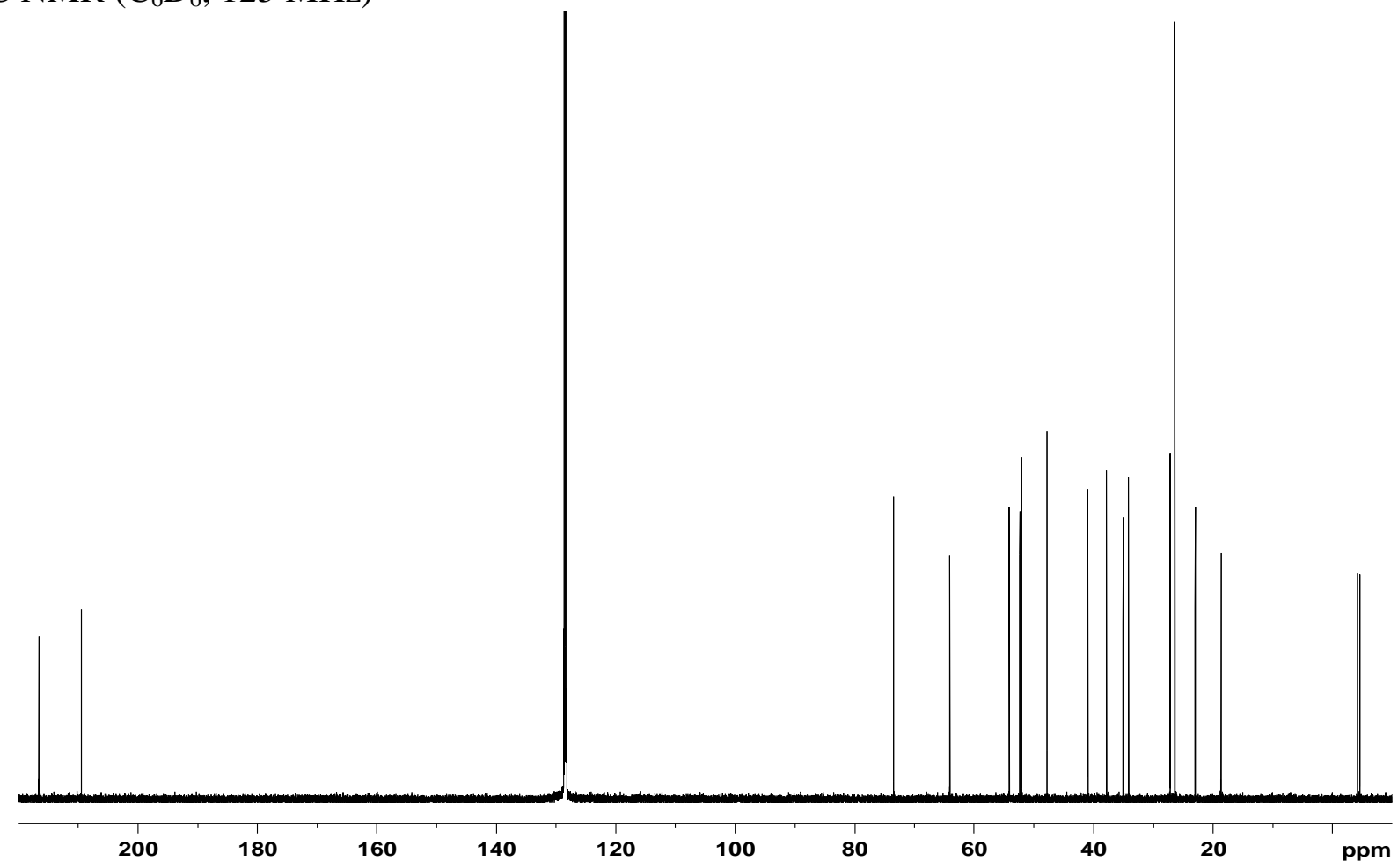


NMR spectra of $\left(1 R^{*}, 2 R^{*}, 3 a^{\prime} R^{*}, 5 R^{*}, 5 \mathrm{a}^{\prime} S^{*}\right)$-5-(tert-butyldimethylsilyl)oxy-2',3',3a',4'-tetrahydro2-vinyl-5' $H$-spiro[cyclopentane-1,1'-pentalene]-2,5'-diol (19)

${ }^{1} \mathrm{H} \mathrm{NMR}\left(\mathrm{CDCl}_{3}, 500 \mathrm{MHz}\right)$
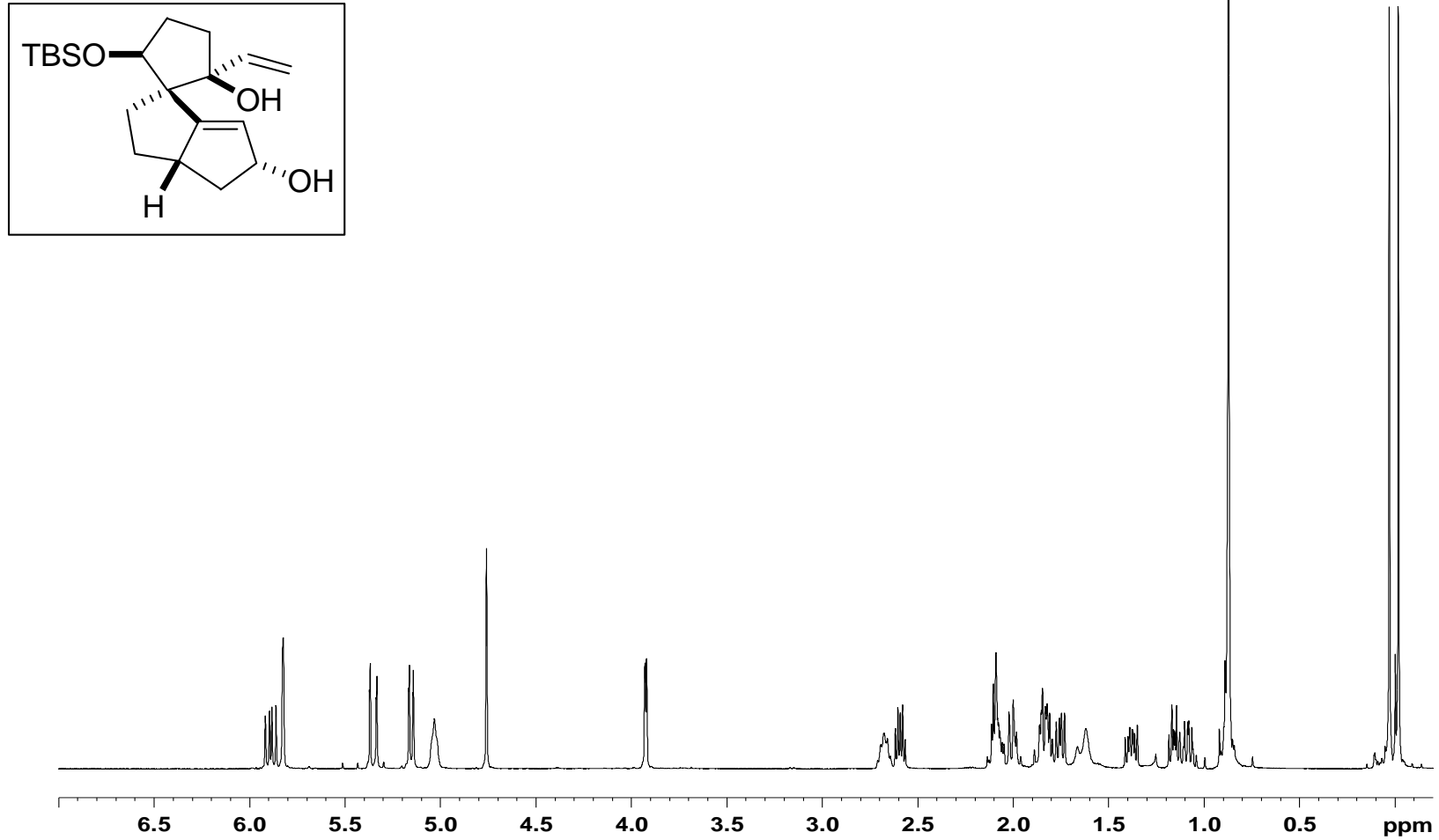

${ }^{13} \mathrm{C} \mathrm{NMR}\left(\mathrm{CDCl}_{3}, 125 \mathrm{MHz}\right)$

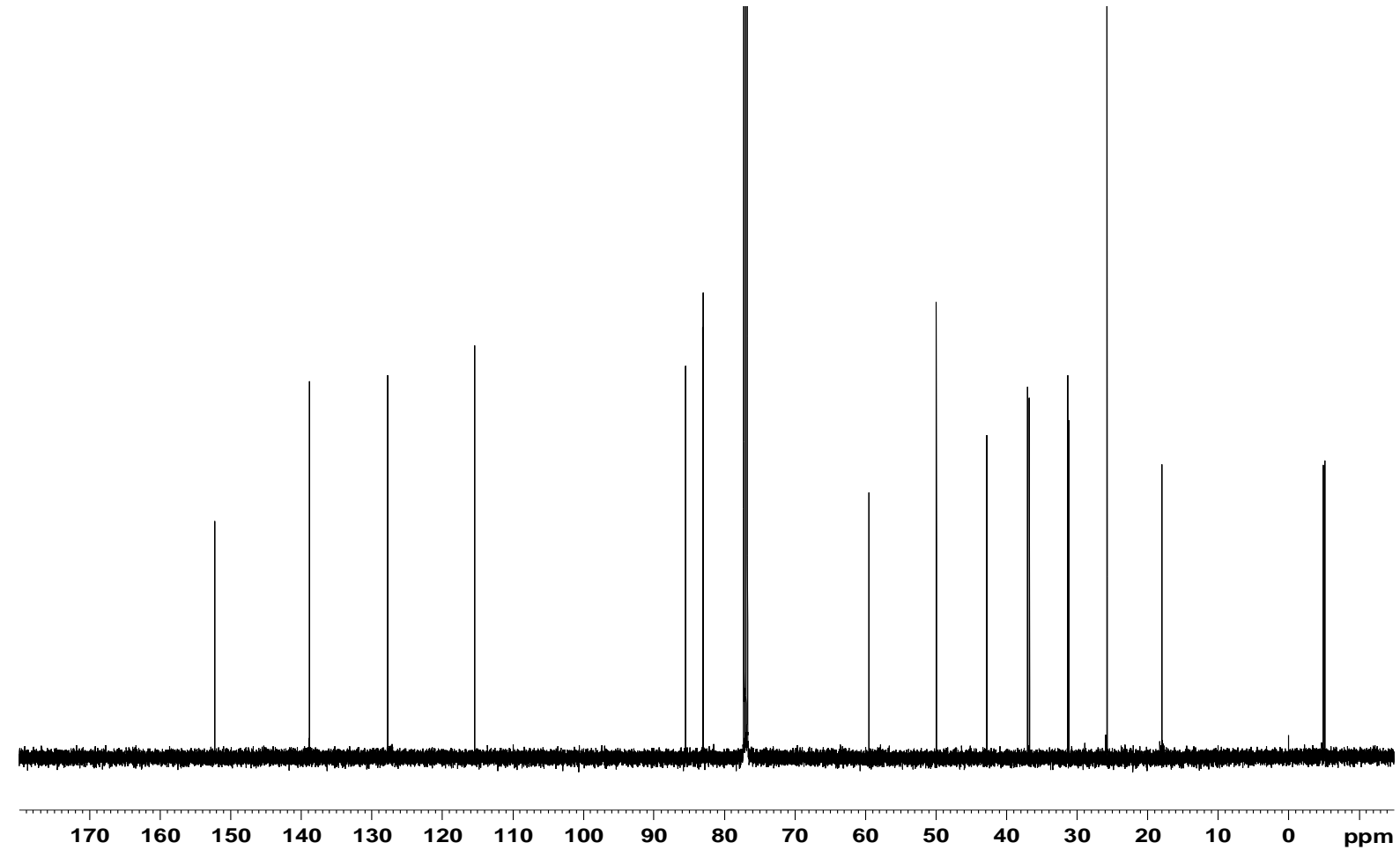


NMR spectra of $\left(1 R^{*}, 2 R^{*}, 3 a^{\prime} R^{*}, 5 R^{*}, 5 \mathrm{a}^{\prime} S^{*}\right)$-5-(tert-butyldimethylsilyl)oxy-2',3',3a',4'-tetrahydro5'-(methoxymethoxy)-2-vinyl-5' $H$-spiro[cyclopentane-1,1'-pentalen]-2-ol (20)

${ }^{1} \mathrm{H} \mathrm{NMR}\left(\mathrm{CDCl}_{3}, 500 \mathrm{MHz}\right)$
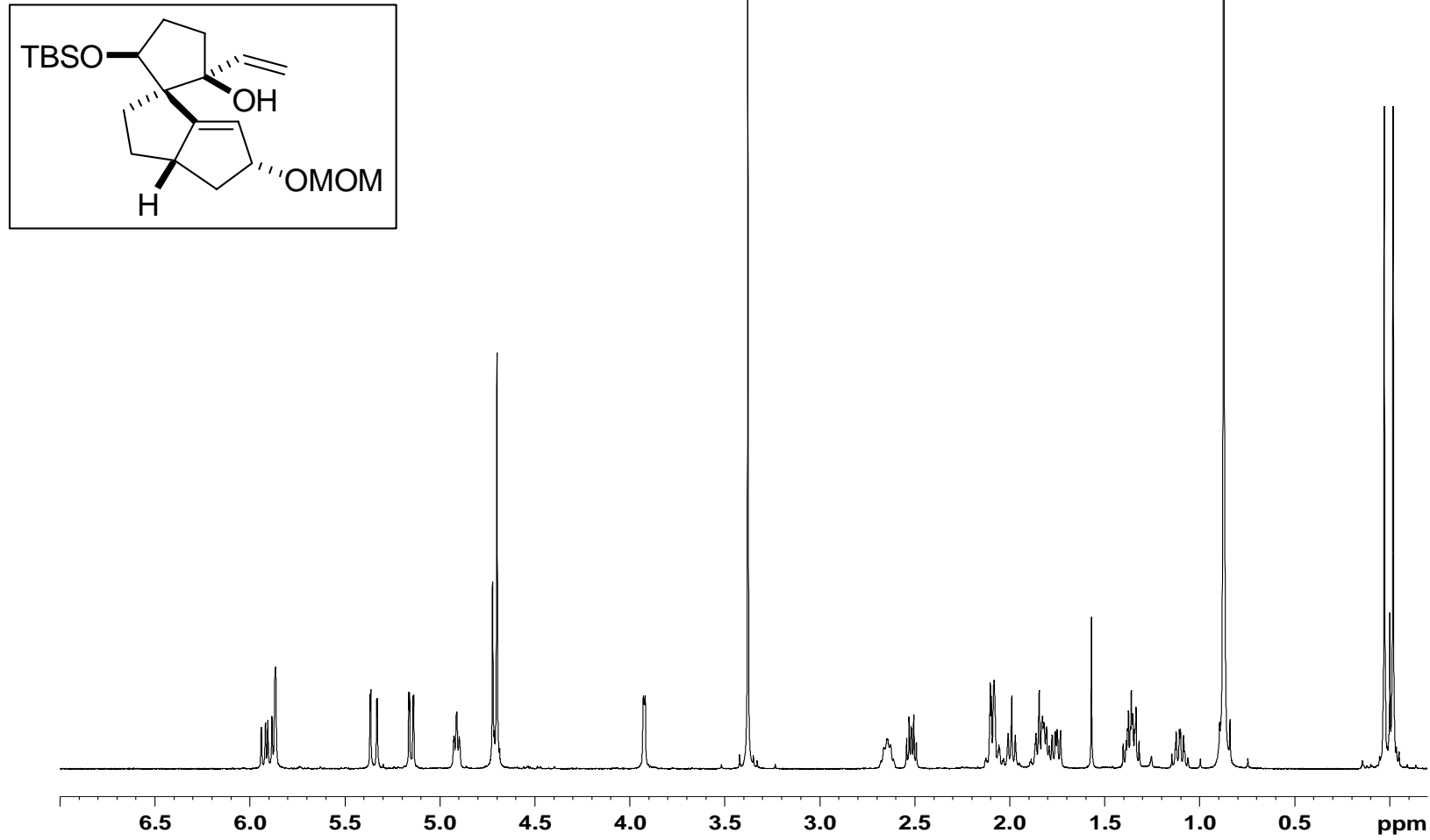

${ }^{13} \mathrm{C} \mathrm{NMR}\left(\mathrm{CDCl}_{3}, 125 \mathrm{MHz}\right)$

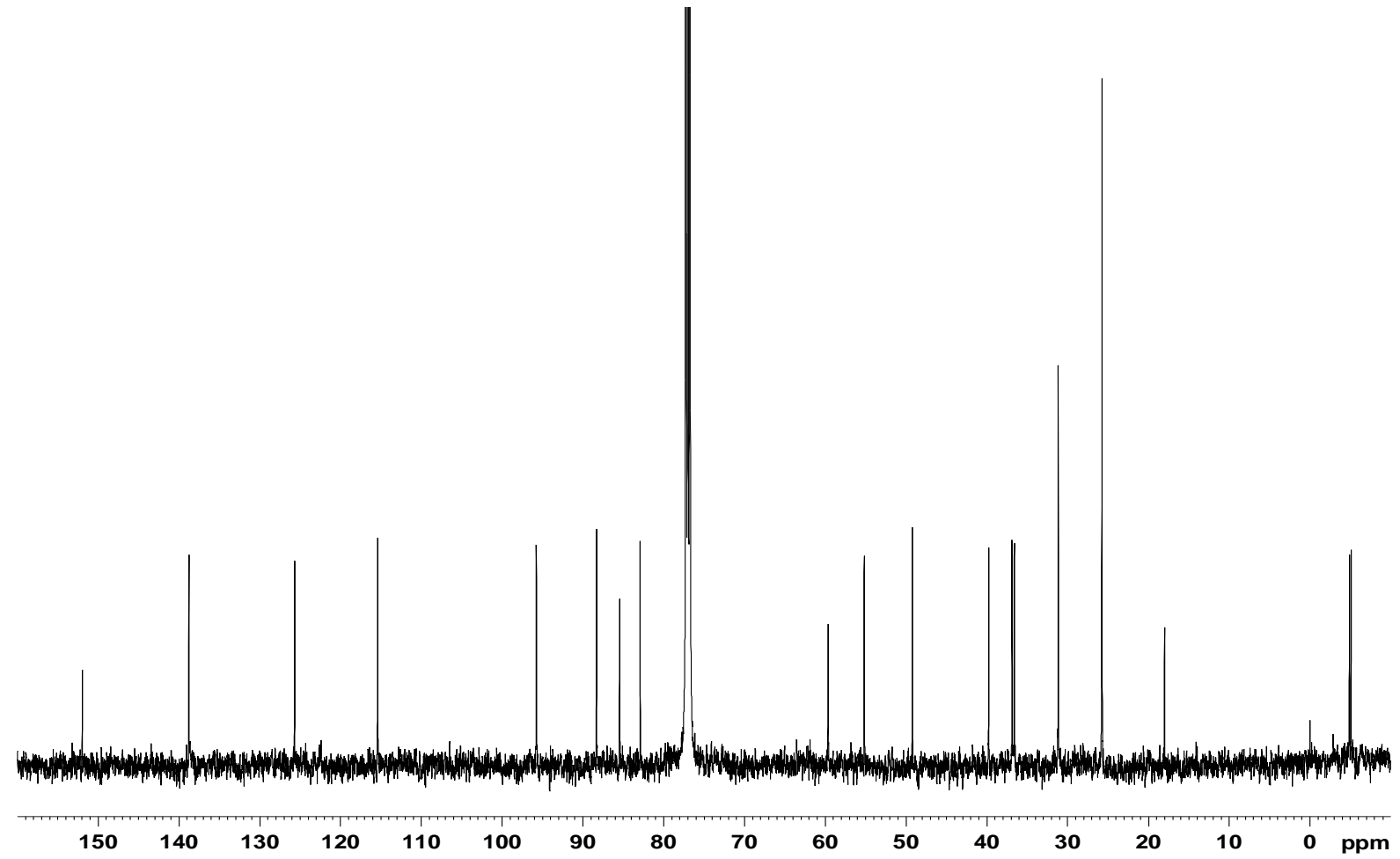


NMR spectra of $\left(4 a R^{*}, 7 R^{*}, 7 a R^{*}, 9 a R^{*}\right)-3,4,4 a, 5,6,7,9,9 a-o c t a h y d r o-4 a, 7$-dihydroxy-1H-cyclopent[kl]-as-indacen-2(8H)-one

${ }^{1} \mathrm{H} \mathrm{NMR}\left(\mathrm{CD}_{3} \mathrm{OD}, 500 \mathrm{MHz}\right)$
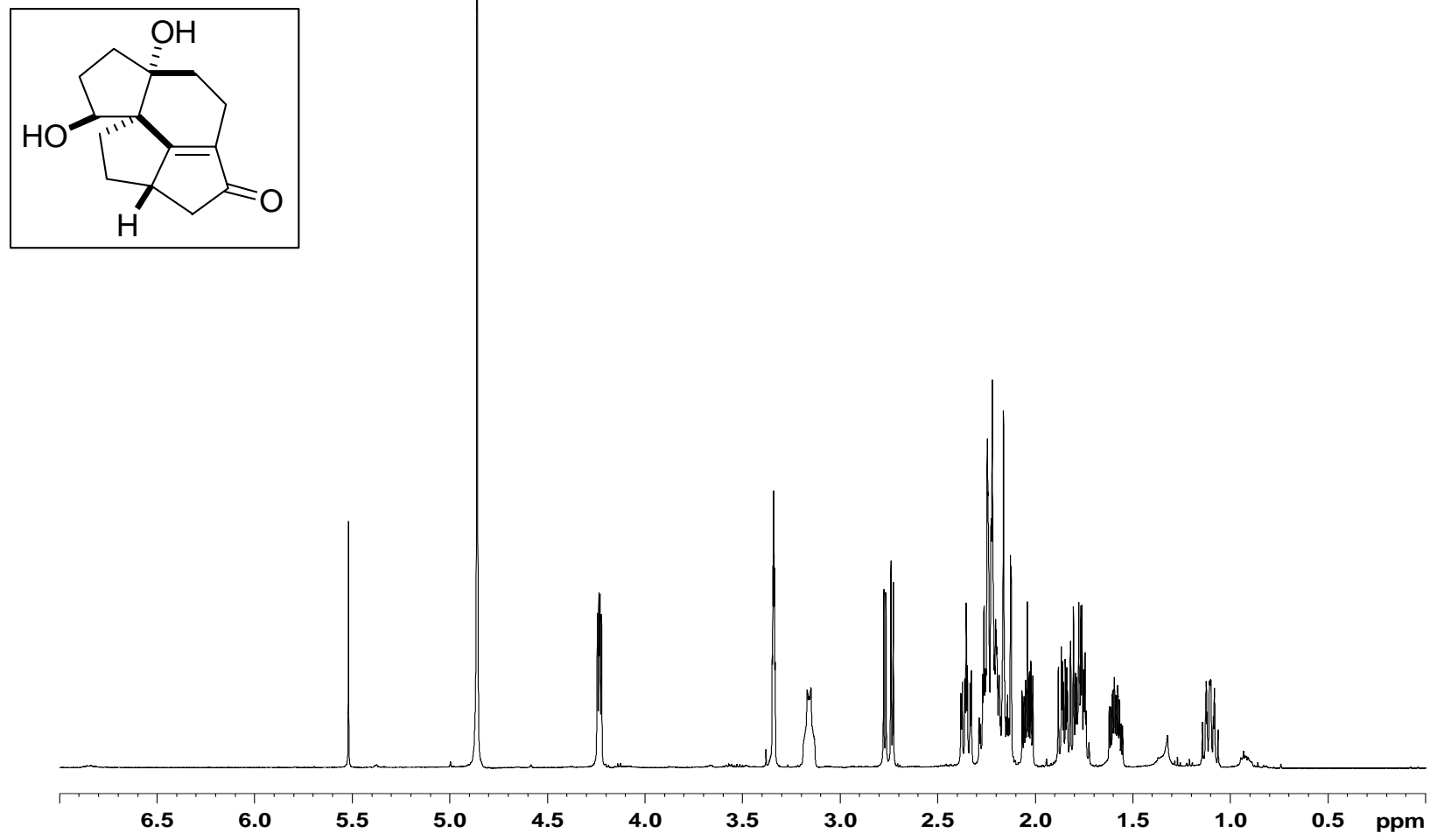

${ }^{13} \mathrm{C}$ NMR $\left(\mathrm{CD}_{3} \mathrm{OD}, 125 \mathrm{MHz}\right)$

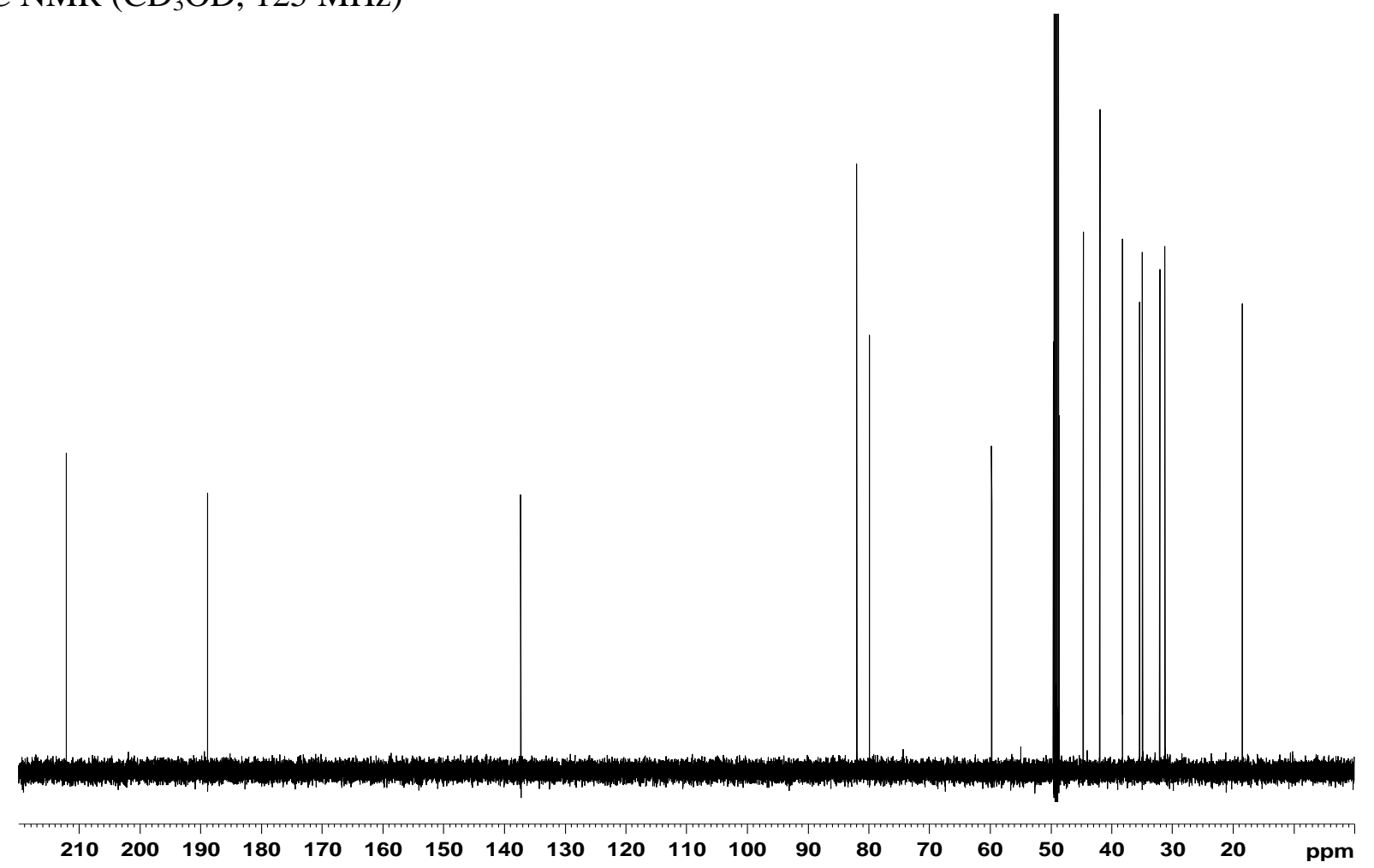


NMR spectra of $\left(4 a R^{*}, 7 R^{*}, 7 a R^{*}, 9 a R^{*}\right)-3,4,4 a, 5,6,7,9,9 a$-octahydro-4a-hydroxy-7-methanesulfonyl-1H-cyclopent $[k l]$-as-indacen-2(8H)-one (23)

${ }^{1} \mathrm{H} \mathrm{NMR}\left(\mathrm{CDCl}_{3}, 500 \mathrm{MHz}\right)$
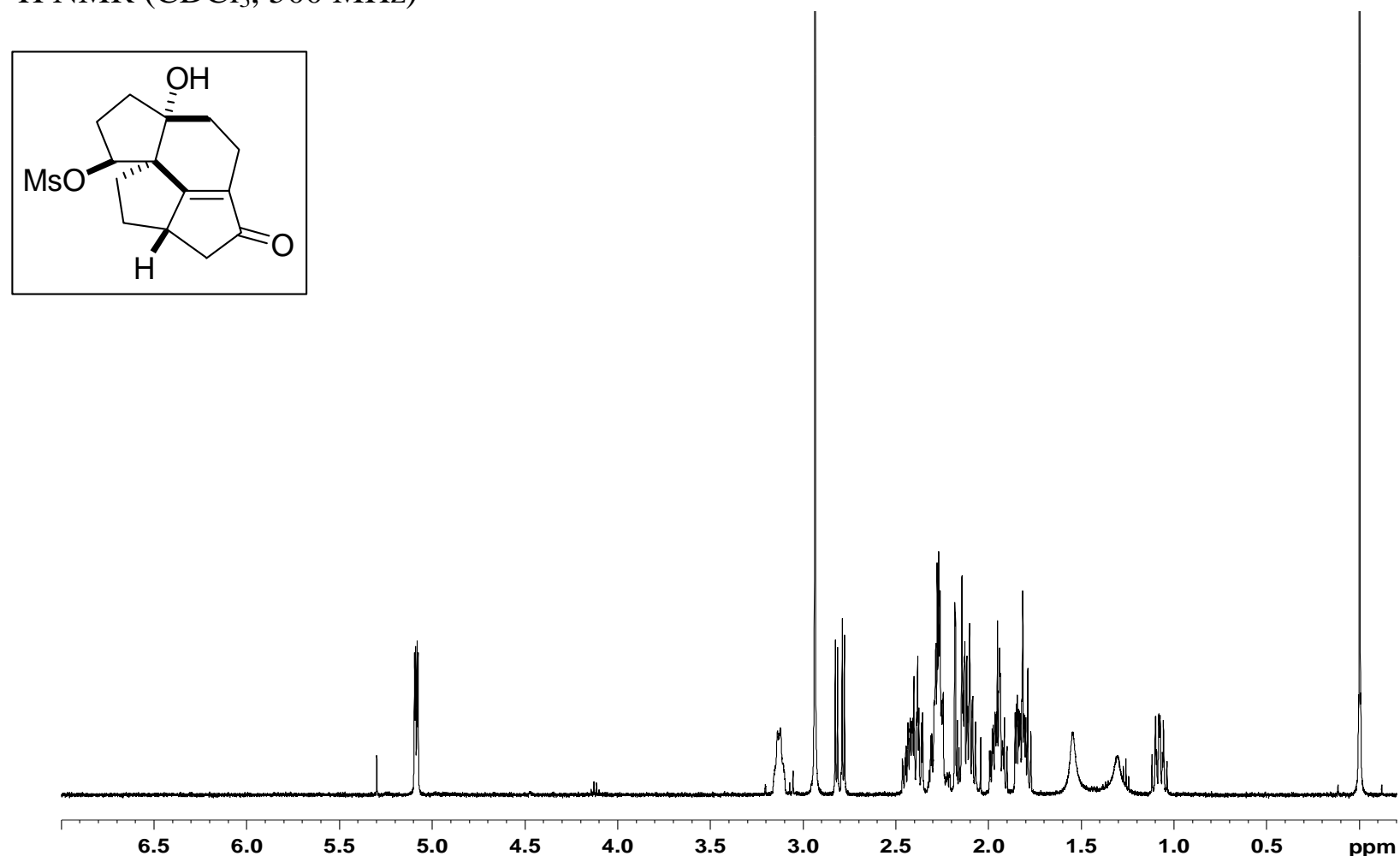

${ }^{13} \mathrm{C} \mathrm{NMR}\left(\mathrm{CDCl}_{3}, 125 \mathrm{MHz}\right)$

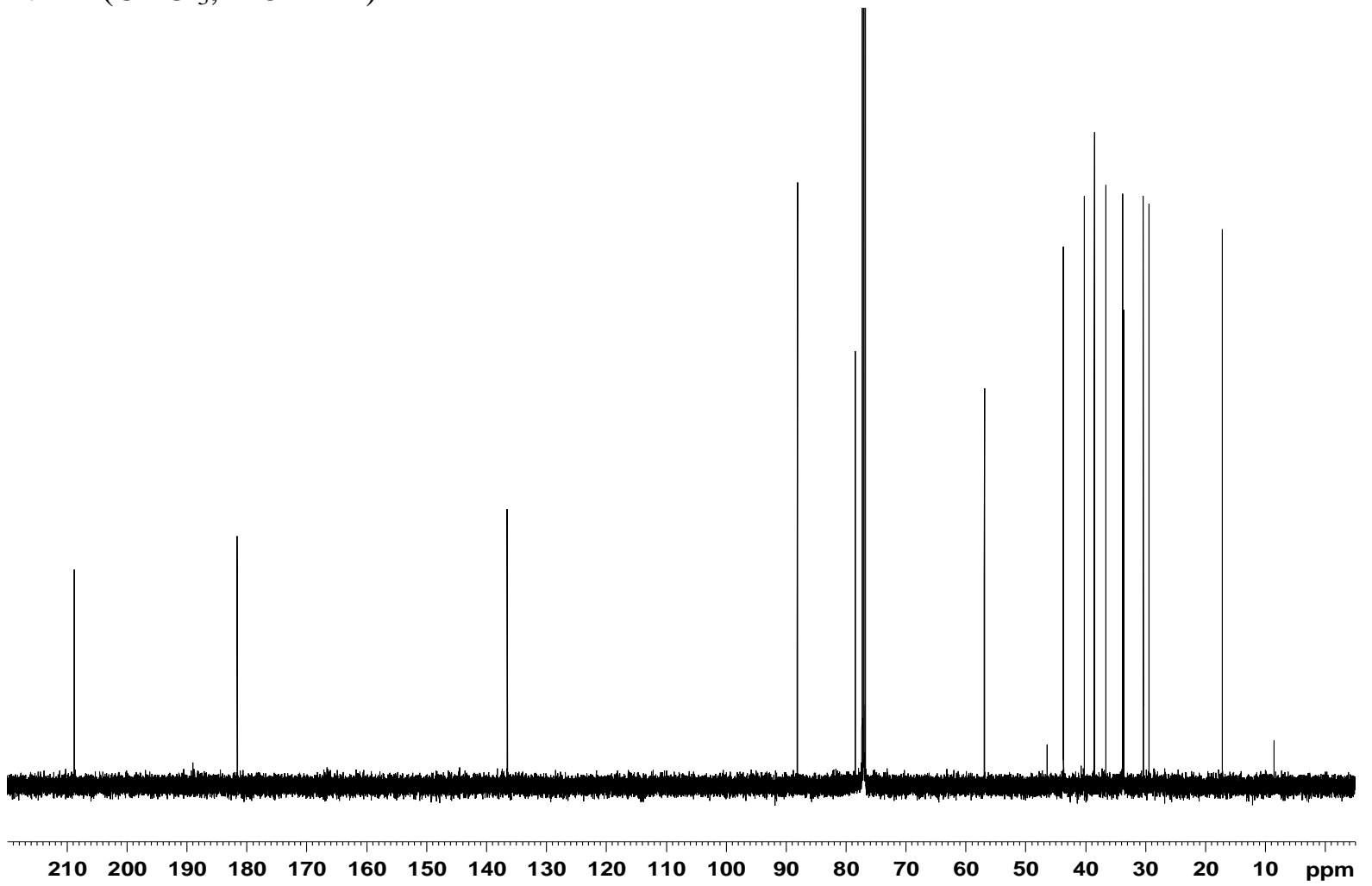


NMR spectra of $\left(2 R^{*}, 4 a S^{*}, 7 S^{*}, 7 a S^{*}, 9 a S^{*}\right)-2,3,4,4 a, 5,6,7,8,9,9 a$-decahydro-7-methanesulfonyl-1Hcyclopent $[k l]$-as-indacene-2,4a-diol (24)

${ }^{1} \mathrm{H}$ NMR $\left(\mathrm{CDCl}_{3}, 500 \mathrm{MHz}\right)$
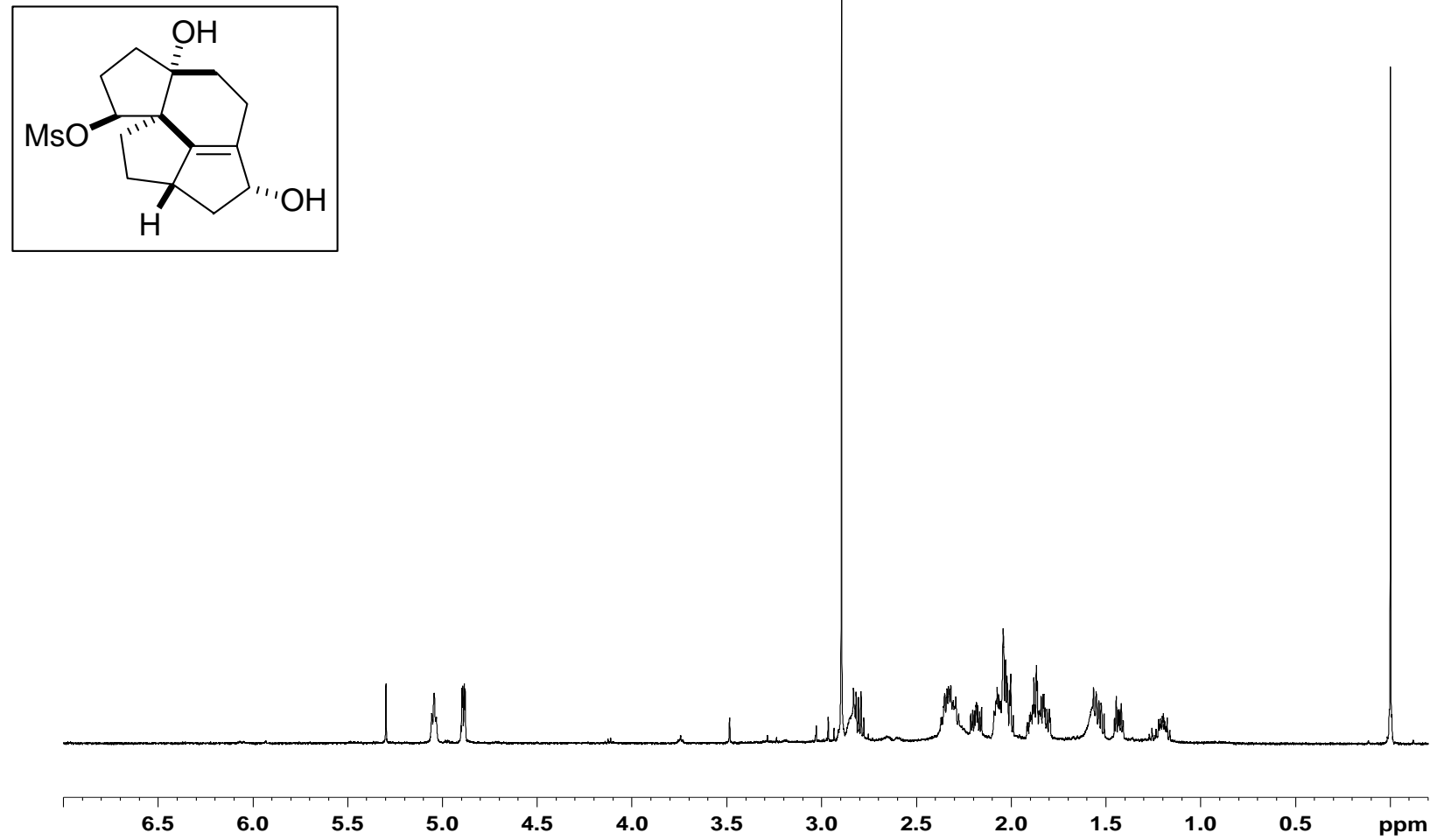

${ }^{13} \mathrm{C} \mathrm{NMR}\left(\mathrm{CDCl}_{3}, 125 \mathrm{MHz}\right)$

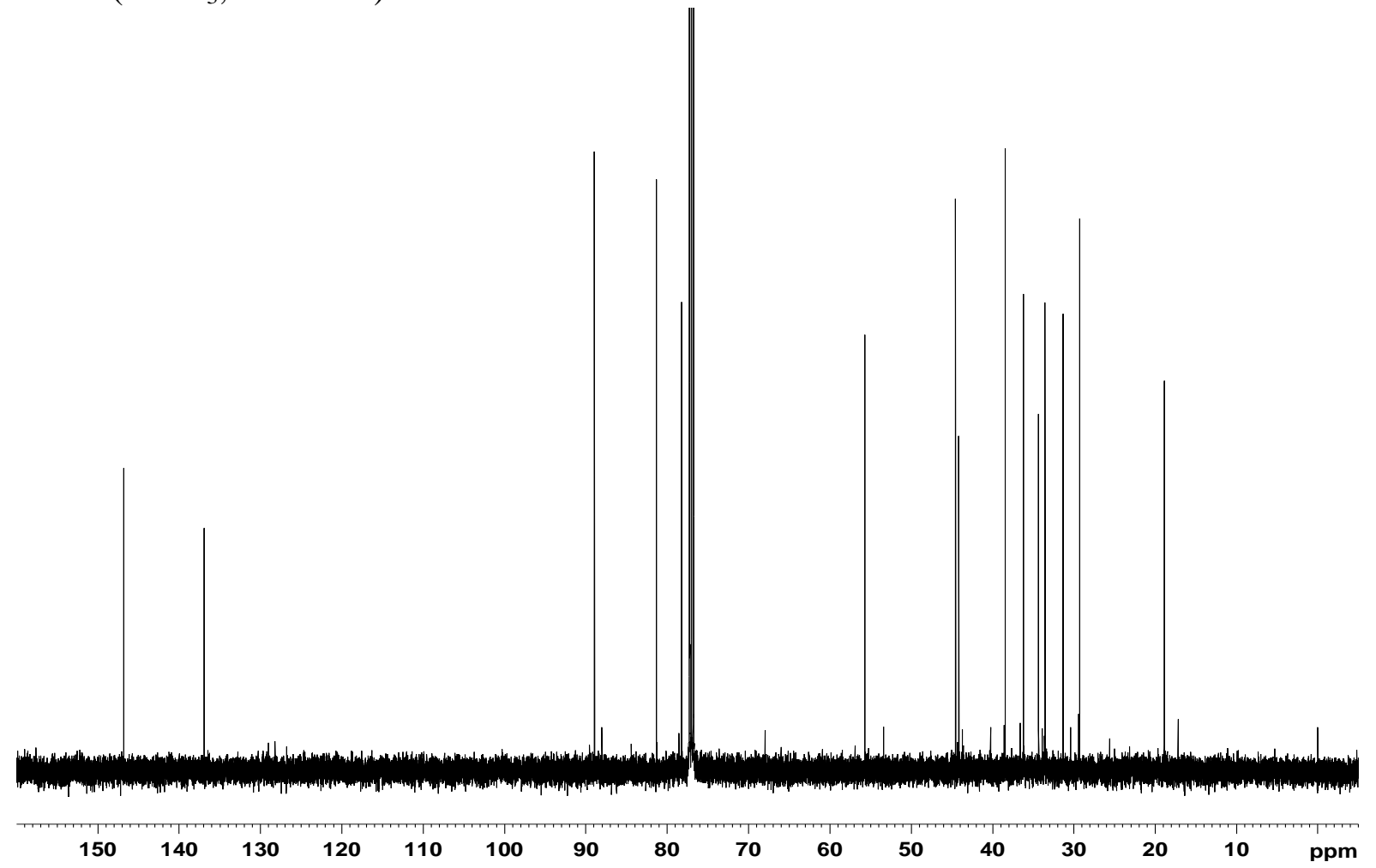


NMR spectra of $\left(2 a R^{*}, 4 S^{*}\right)-2,2 a, 3,4,5,6,8,9-o c t a h y d r o-4-h y d r o x y c y c l o n o n a[c d]$ pentalen-7(1H)-one (25)

${ }^{1} \mathrm{H} \mathrm{NMR}\left(\mathrm{CDCl}_{3}, 500 \mathrm{MHz}\right)$
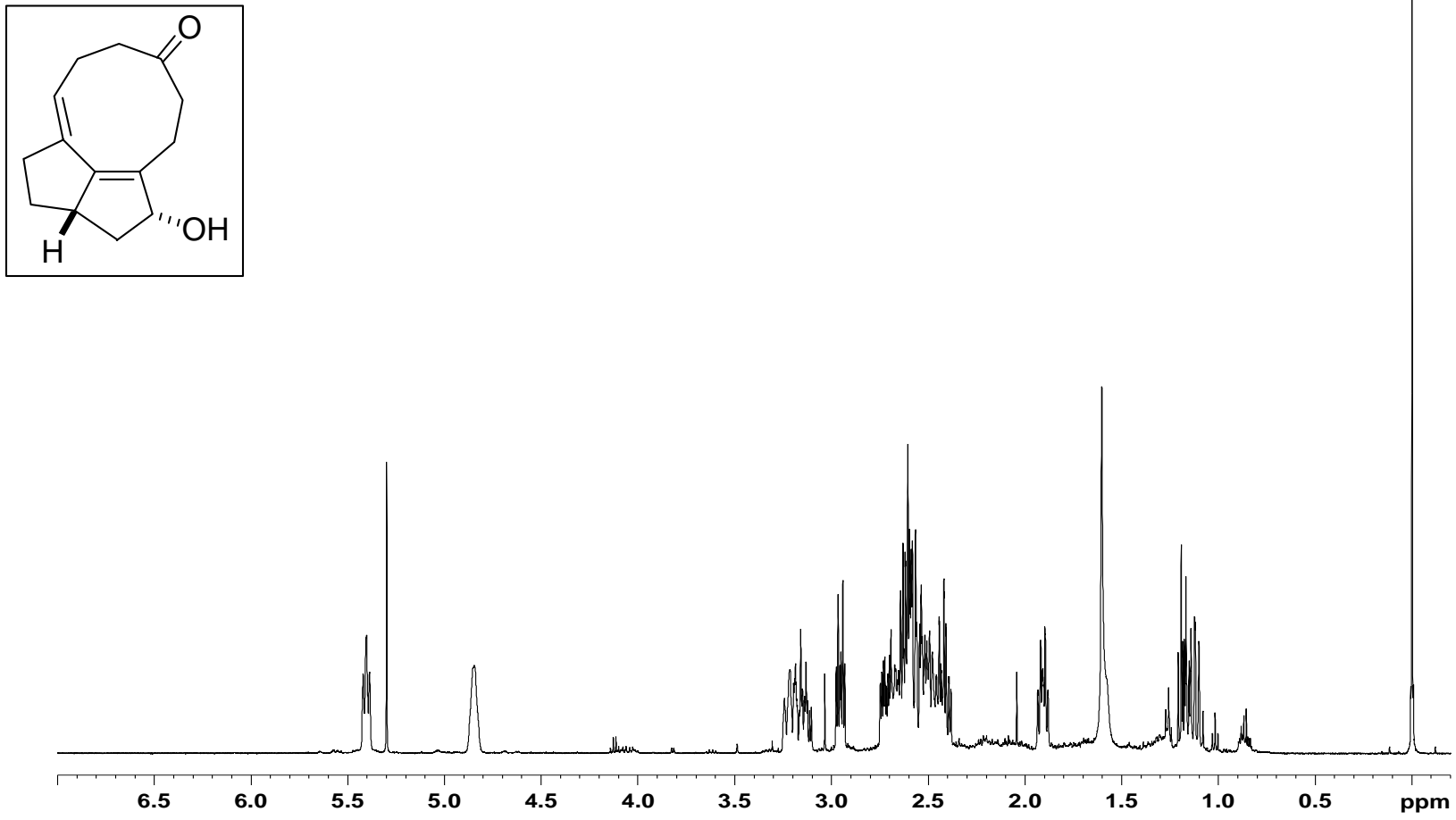

${ }^{13} \mathrm{C} \mathrm{NMR}\left(\mathrm{CDCl}_{3}, 125 \mathrm{MHz}\right)$

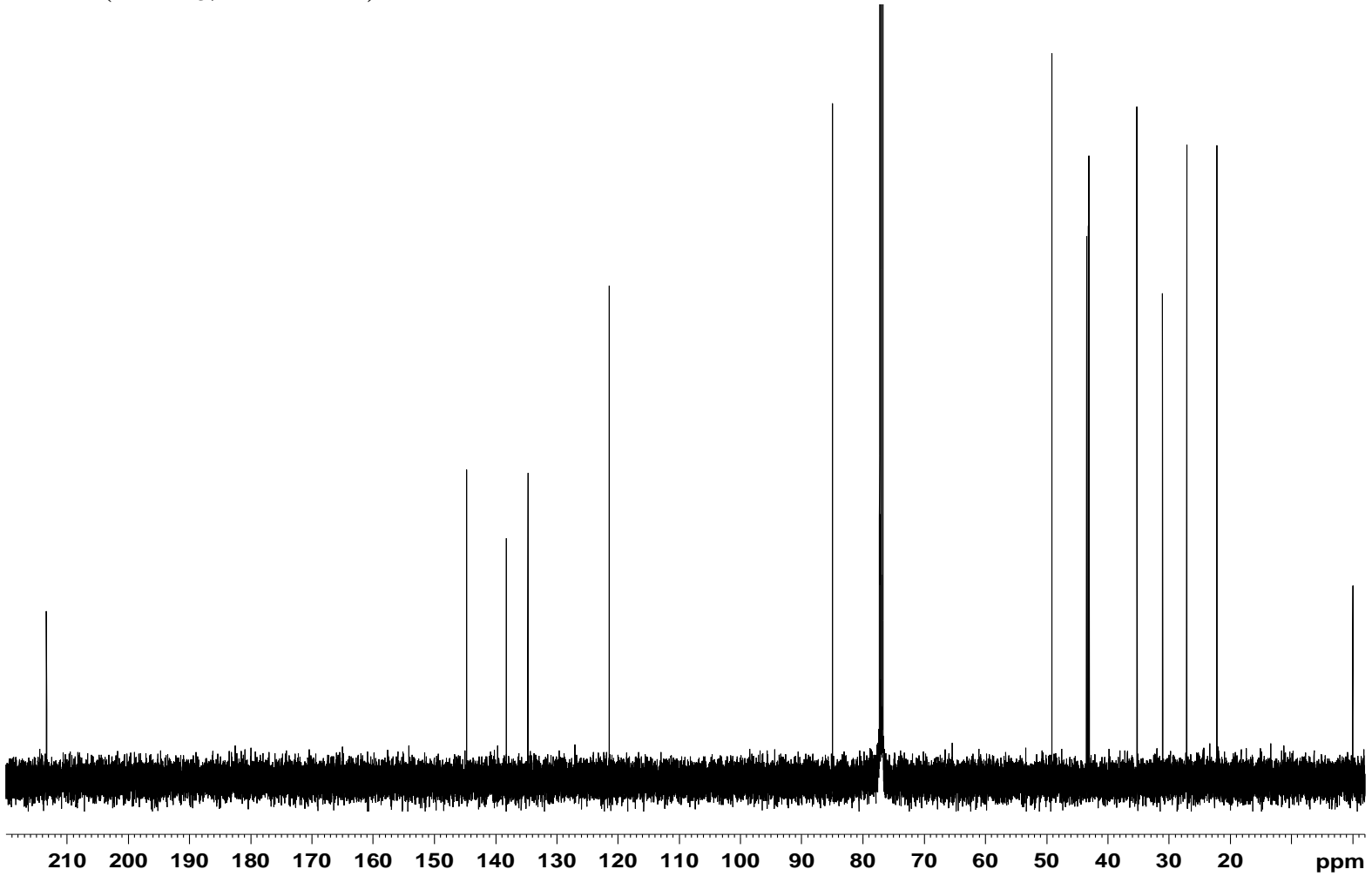


NMR spectra of $\left(2 R^{*}, 4 a S^{*}, 7 S^{*}, 7 a S^{*}, 9 a S^{*}\right)-2,3,4,4 a, 5,6,7,8,9,9 a$-decahydro-7-methanesulfonyl-2(methoxymethyl)-1H-cyclopent $[\mathrm{kl}]$-as-indacen-4a-ol (26)

${ }^{1} \mathrm{H} \mathrm{NMR}\left(\mathrm{CDCl}_{3}, 500 \mathrm{MHz}\right)$
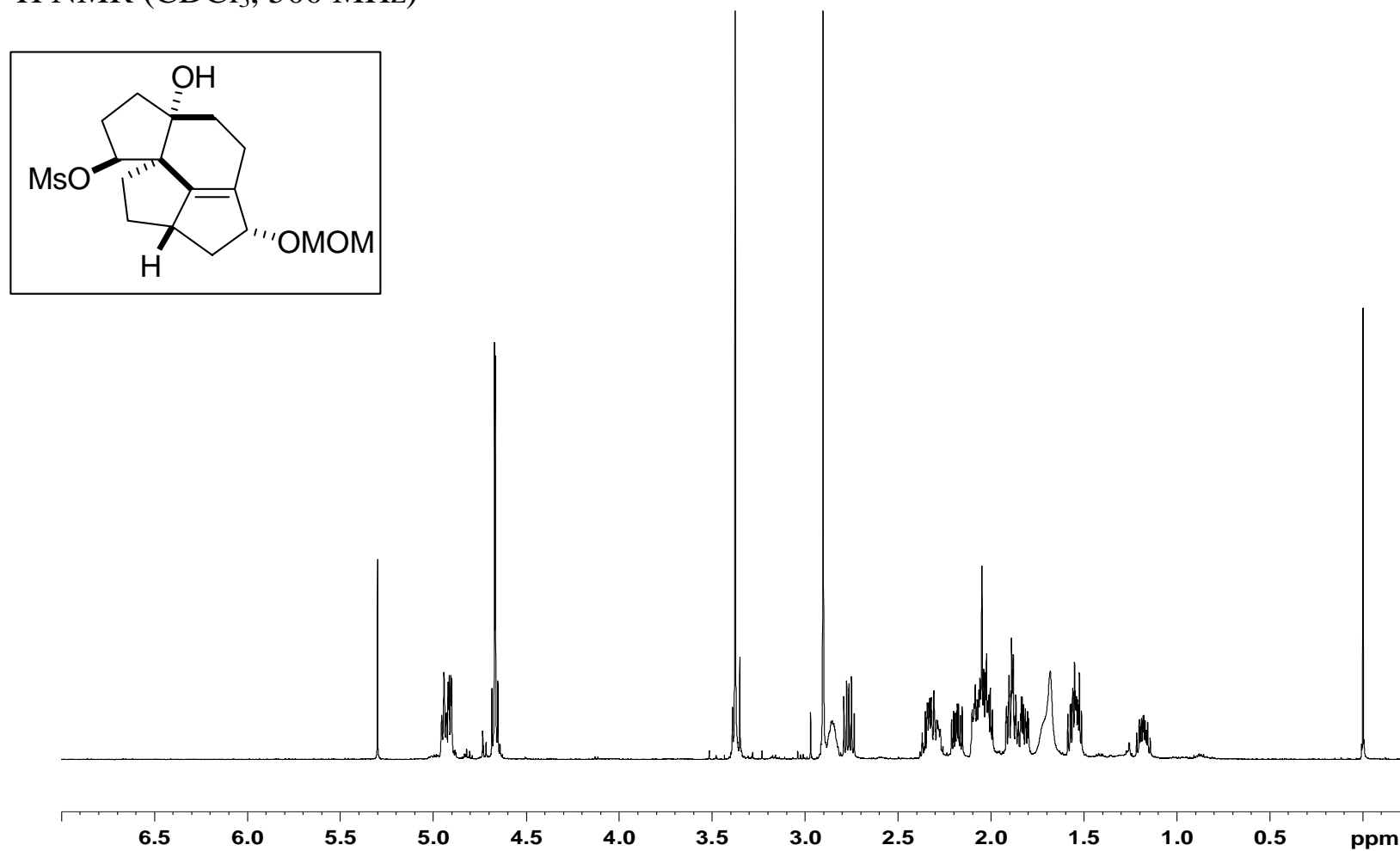

${ }^{13} \mathrm{C} \mathrm{NMR}\left(\mathrm{CDCl}_{3}, 125 \mathrm{MHz}\right)$

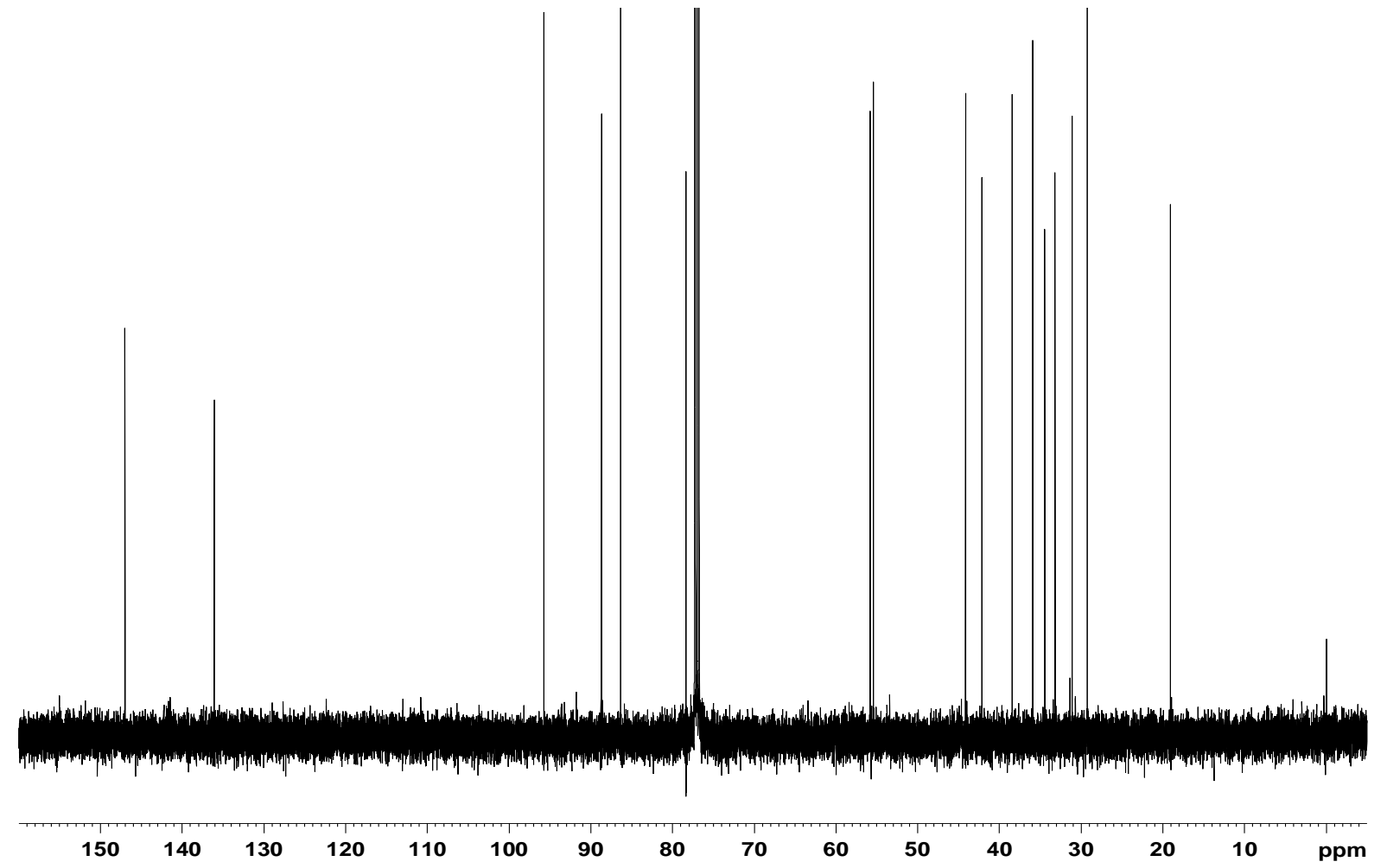




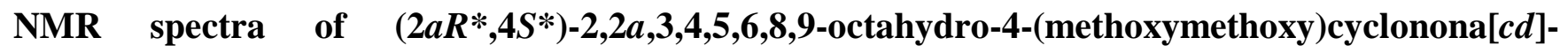
pentalen-7(1H)-one $(27)$

${ }^{1} \mathrm{H} \mathrm{NMR}\left(\mathrm{CDCl}_{3}, 500 \mathrm{MHz}\right)$
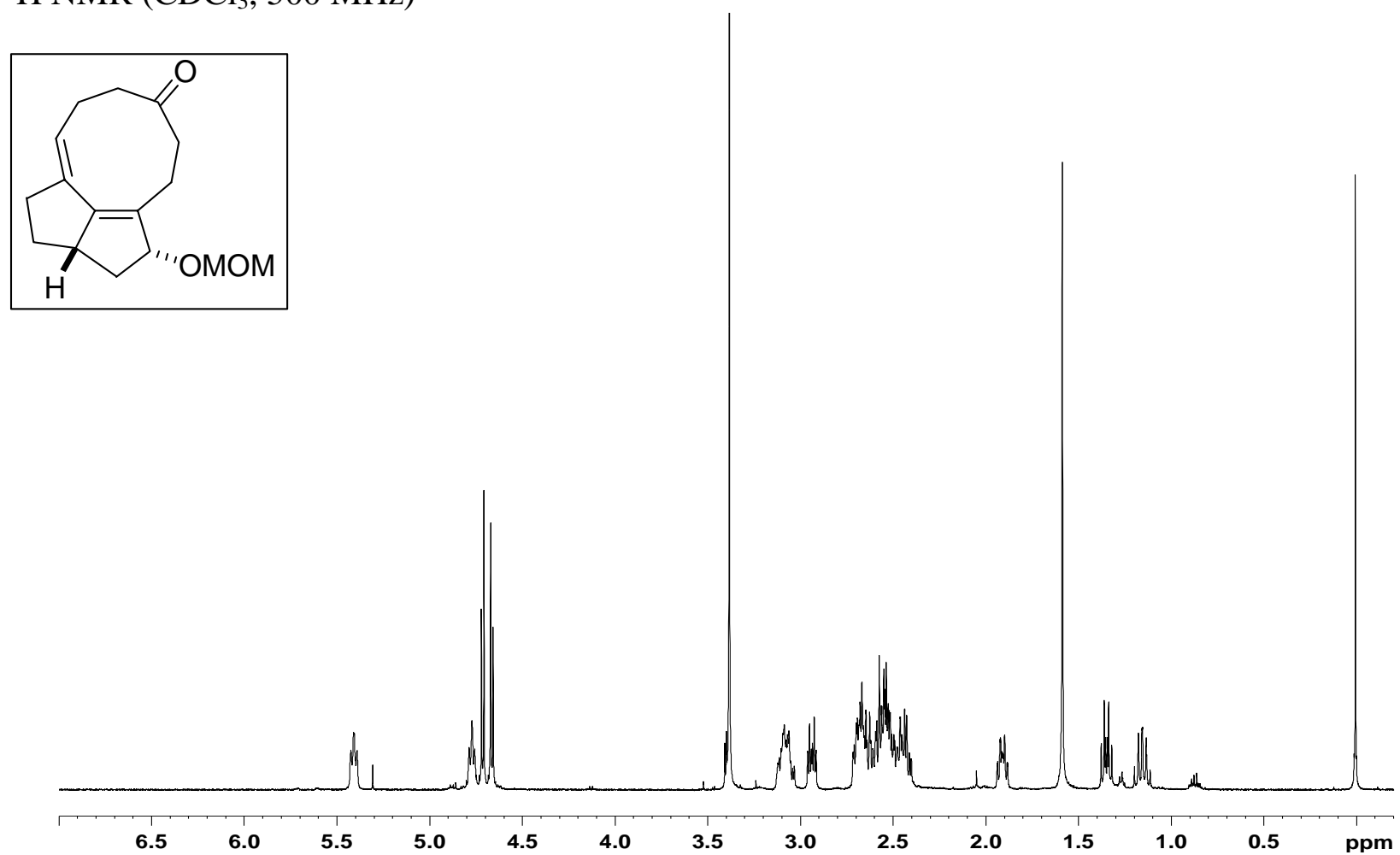

${ }^{13} \mathrm{C} \mathrm{NMR}\left(\mathrm{CDCl}_{3}, 125 \mathrm{MHz}\right)$

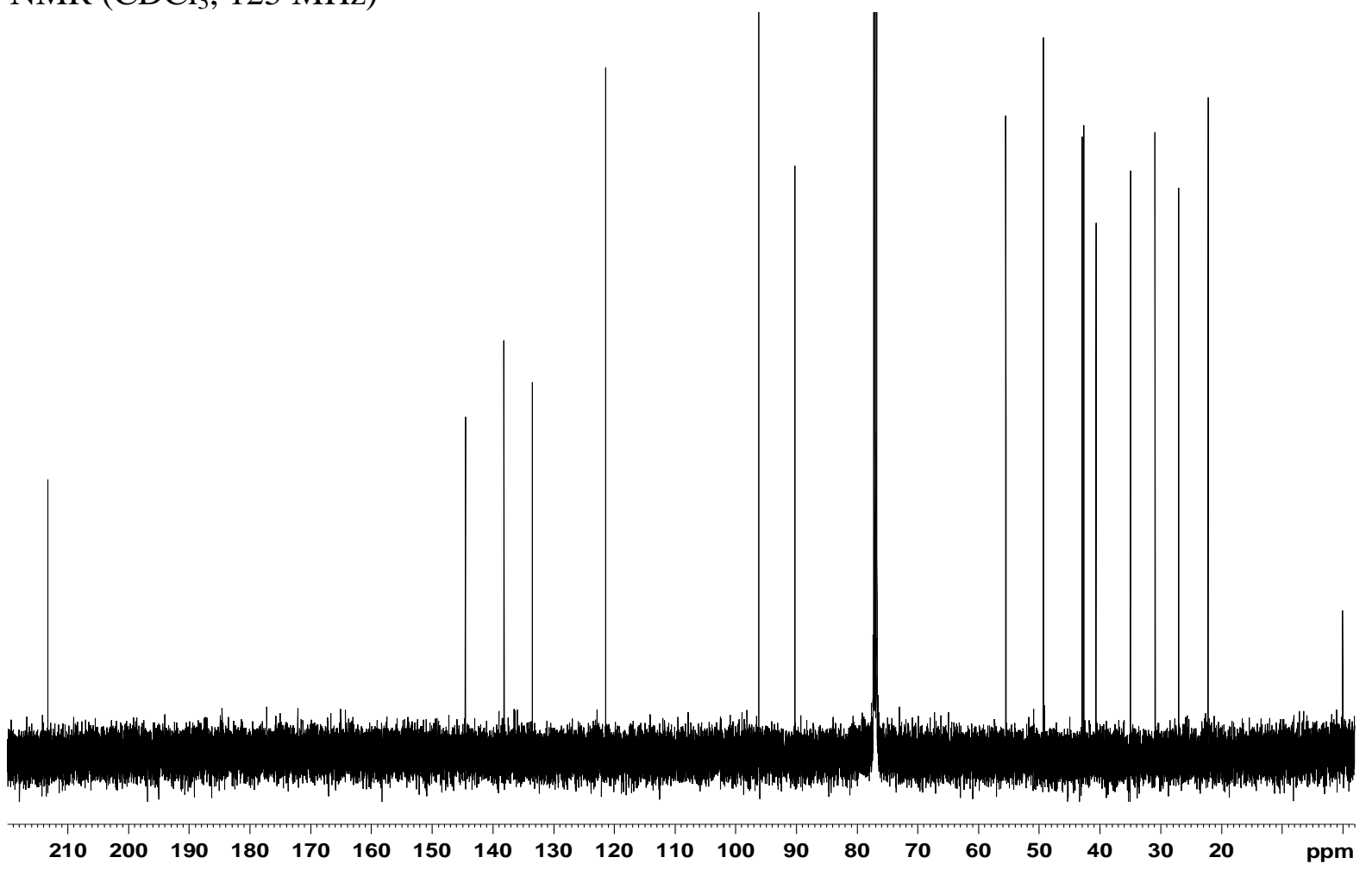


NMR spectra of $\left(2 R^{*}, 3 S^{*}\right)-2$-(but-3-enyl)-3-(tert-butyldimethylsilyl)oxy-2-((trimethylsilyl)ethynyl)cyclopentanone

${ }^{1} \mathrm{H}$ NMR $\left(\mathrm{CDCl}_{3}, 500 \mathrm{MHz}\right)$
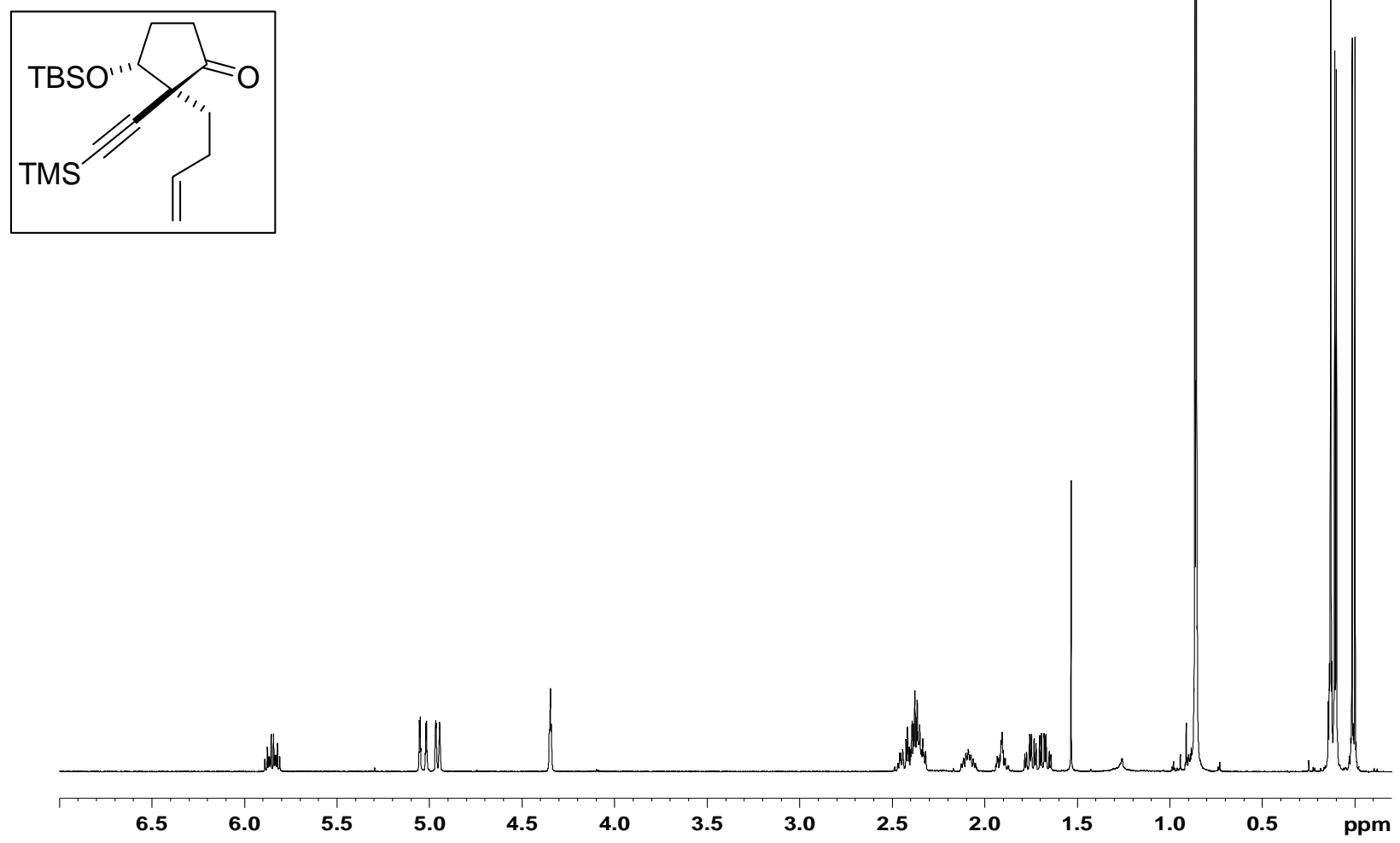

${ }^{13} \mathrm{C} \mathrm{NMR}\left(\mathrm{CDCl}_{3}, 125 \mathrm{MHz}\right)$

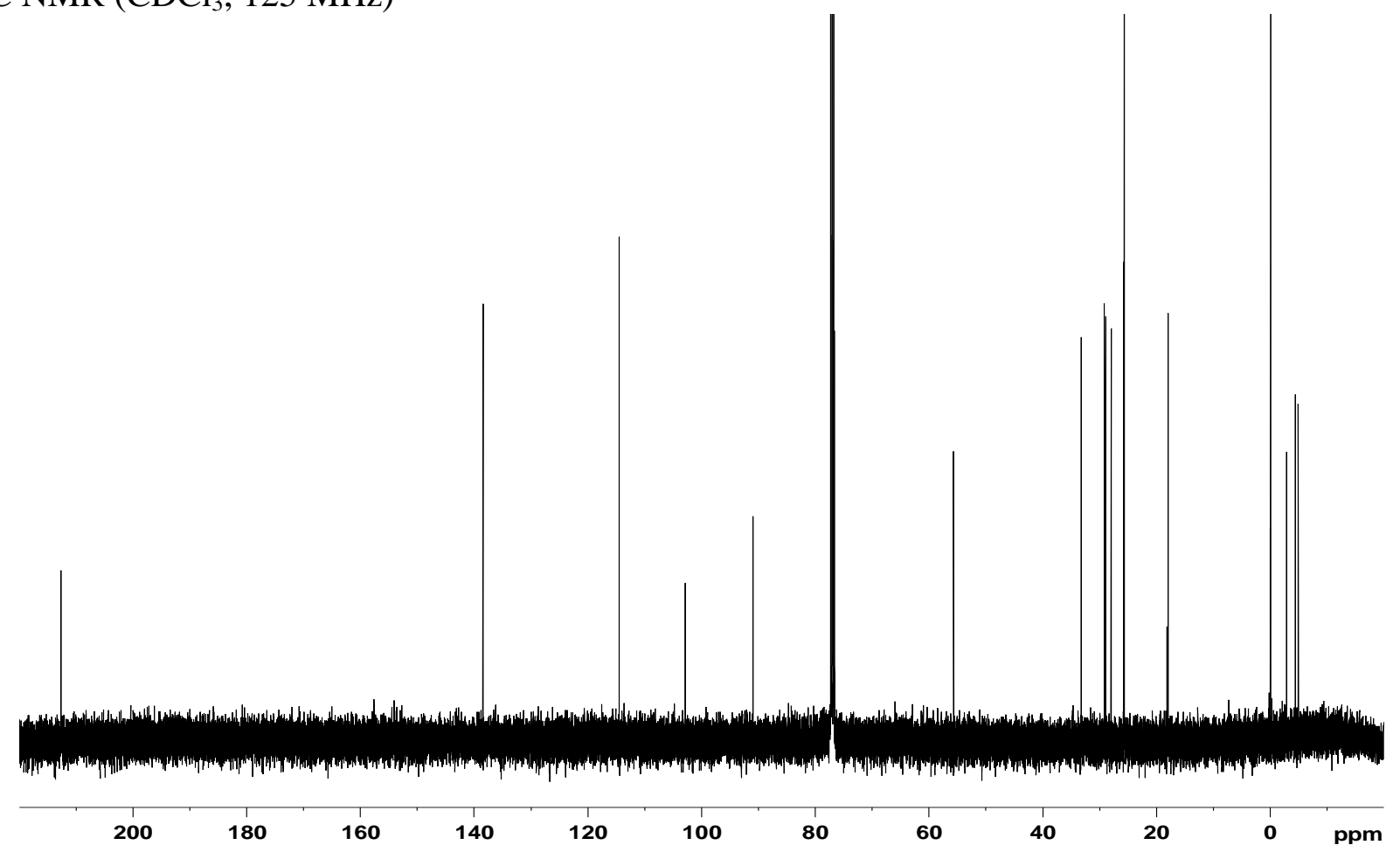


NMR spectra of $\left(1 R *, 2 S * 3 S^{*}\right)$-2-(but-3-enyl)-3-(tert-butyldimethylsilyl)oxy-2-((trimethylsilyl)ethynyl)-1-vinylcyclopentanol

${ }^{1} \mathrm{H} \mathrm{NMR}\left(\mathrm{CDCl}_{3}, 500 \mathrm{MHz}\right)$
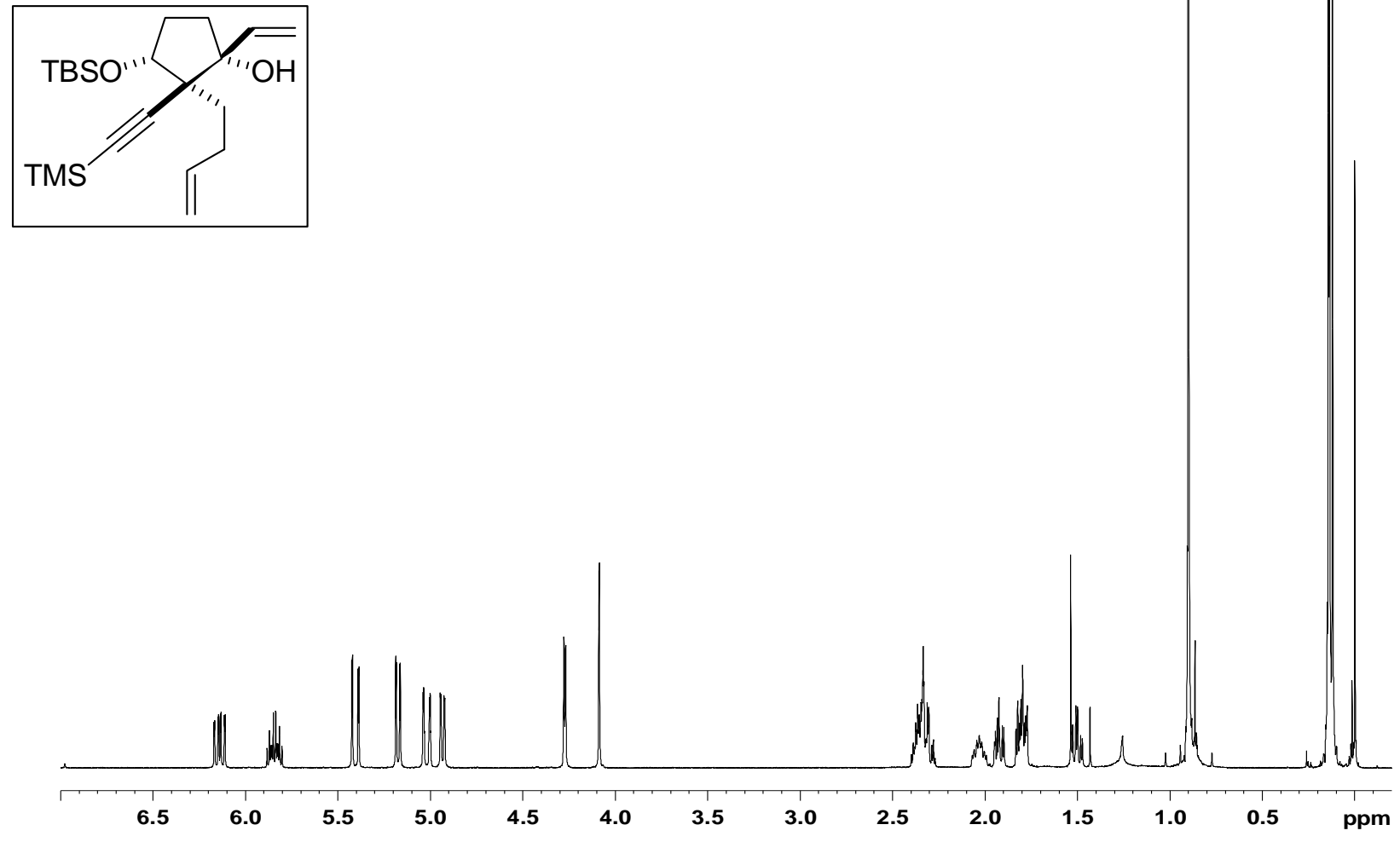

${ }^{13} \mathrm{C} \mathrm{NMR}\left(\mathrm{CDCl}_{3}, 125 \mathrm{MHz}\right)$

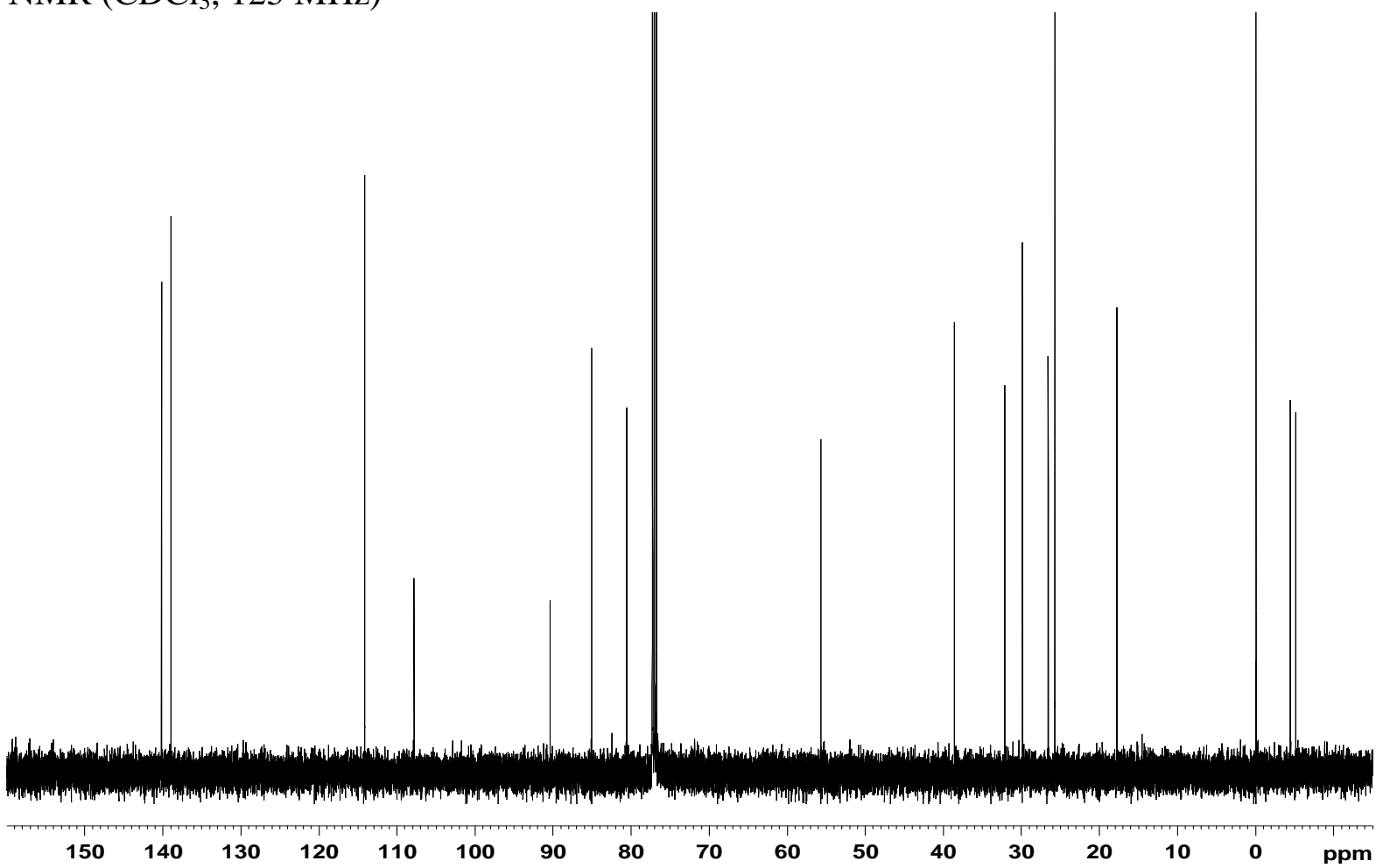


NMR spectra of $\left(1 S^{*}, 2 R^{*}, 3 S^{*}\right)$-2-(but-3-enyl)-3-(tert-butyldimethylsilyl)oxy-2-ethynyl-1-vinylcyclopentan-1-ol (7)

${ }^{1} \mathrm{H} \mathrm{NMR}\left(\mathrm{CDCl}_{3}, 500 \mathrm{MHz}\right)$
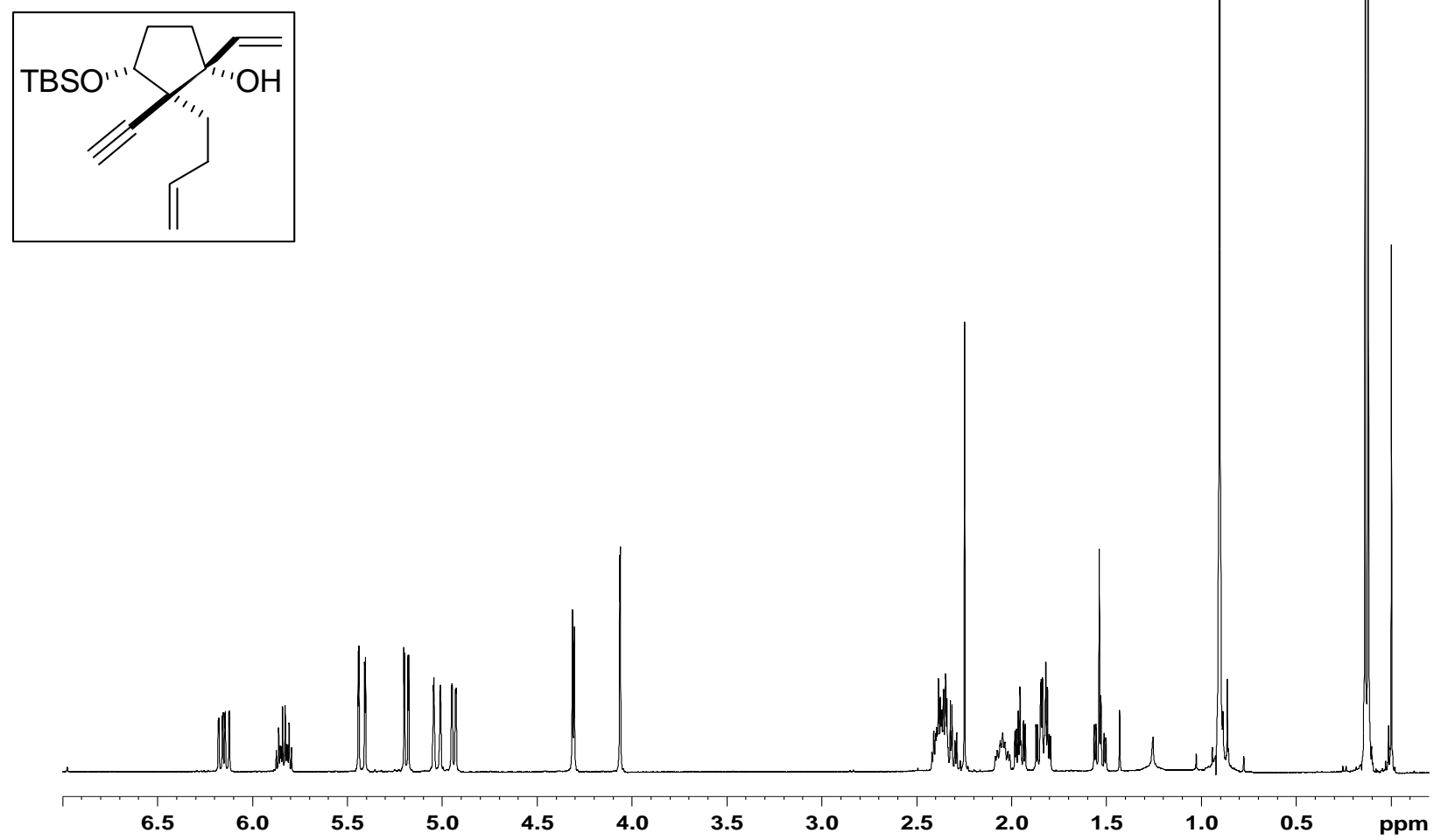

${ }^{13} \mathrm{C} \mathrm{NMR}\left(\mathrm{CDCl}_{3}, 125 \mathrm{MHz}\right)$

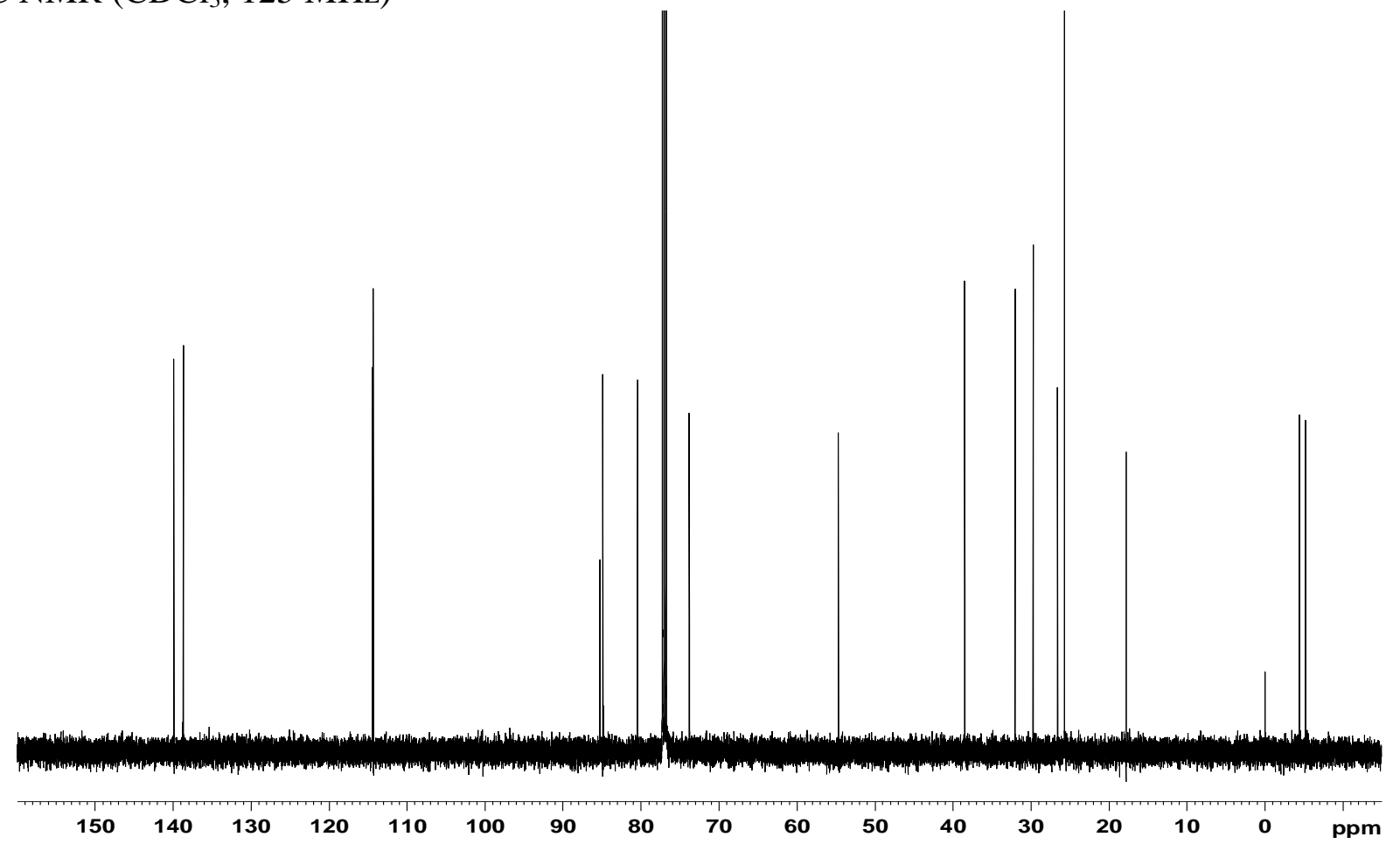


NMR spectra of $\left(1 R^{*}, 2 S^{*}, 3 a^{\prime} R^{*}, 5 S^{*}, 5 \mathrm{a}^{\prime} S^{*}\right)$-5-(tert-butyldimethylsilyl)oxy-2',3',3$a^{\prime}, 4^{\prime}$-tetrahydro2-vinyl-5' $H$-spiro[cyclopentane-1,1'-pentalene]-2,5'-diol (28)

${ }^{1} \mathrm{H} \mathrm{NMR}\left(\mathrm{CDCl}_{3}, 500 \mathrm{MHz}\right)$
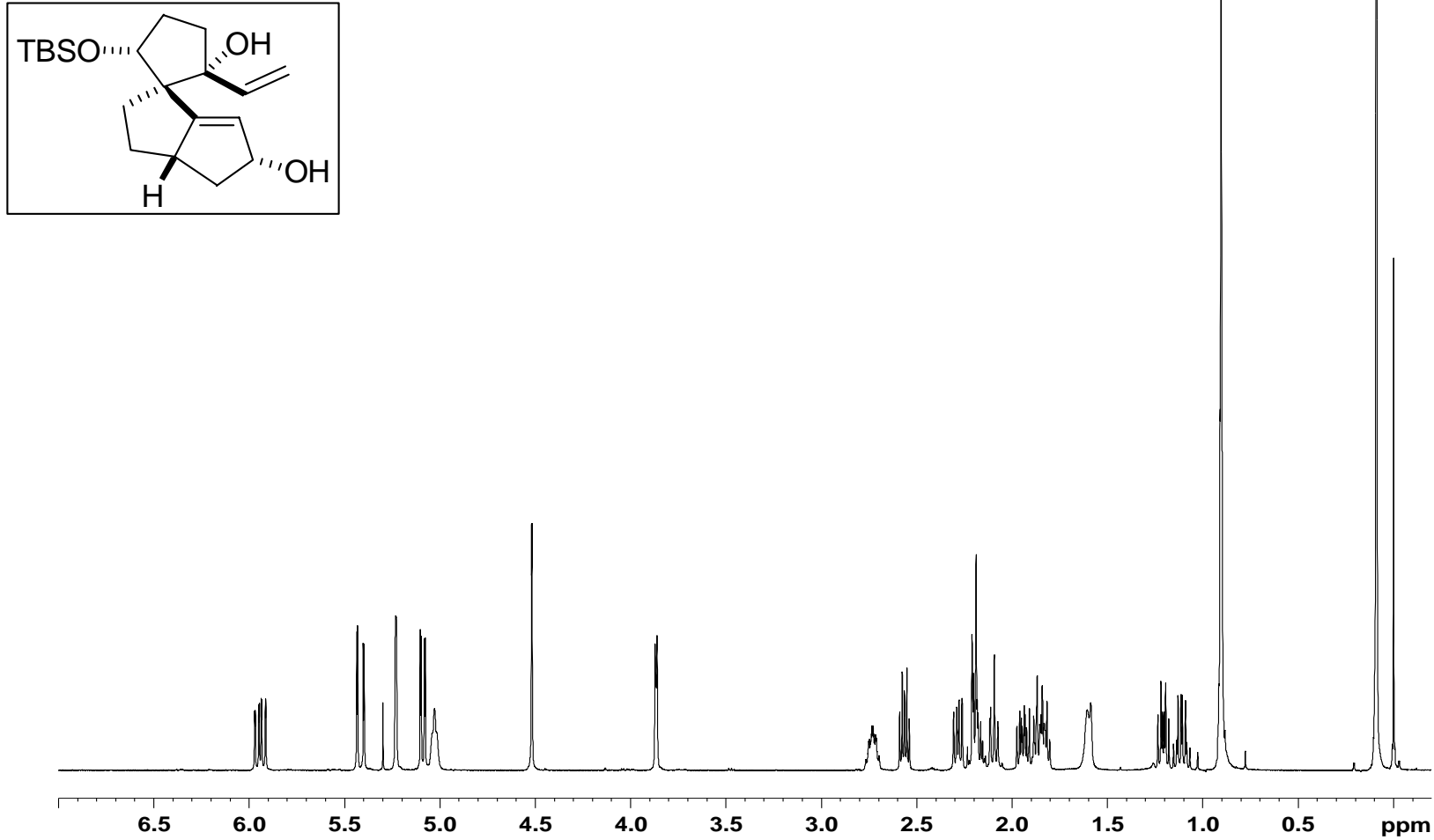

${ }^{13} \mathrm{C} \mathrm{NMR}\left(\mathrm{CDCl}_{3}, 125 \mathrm{MHz}\right)$

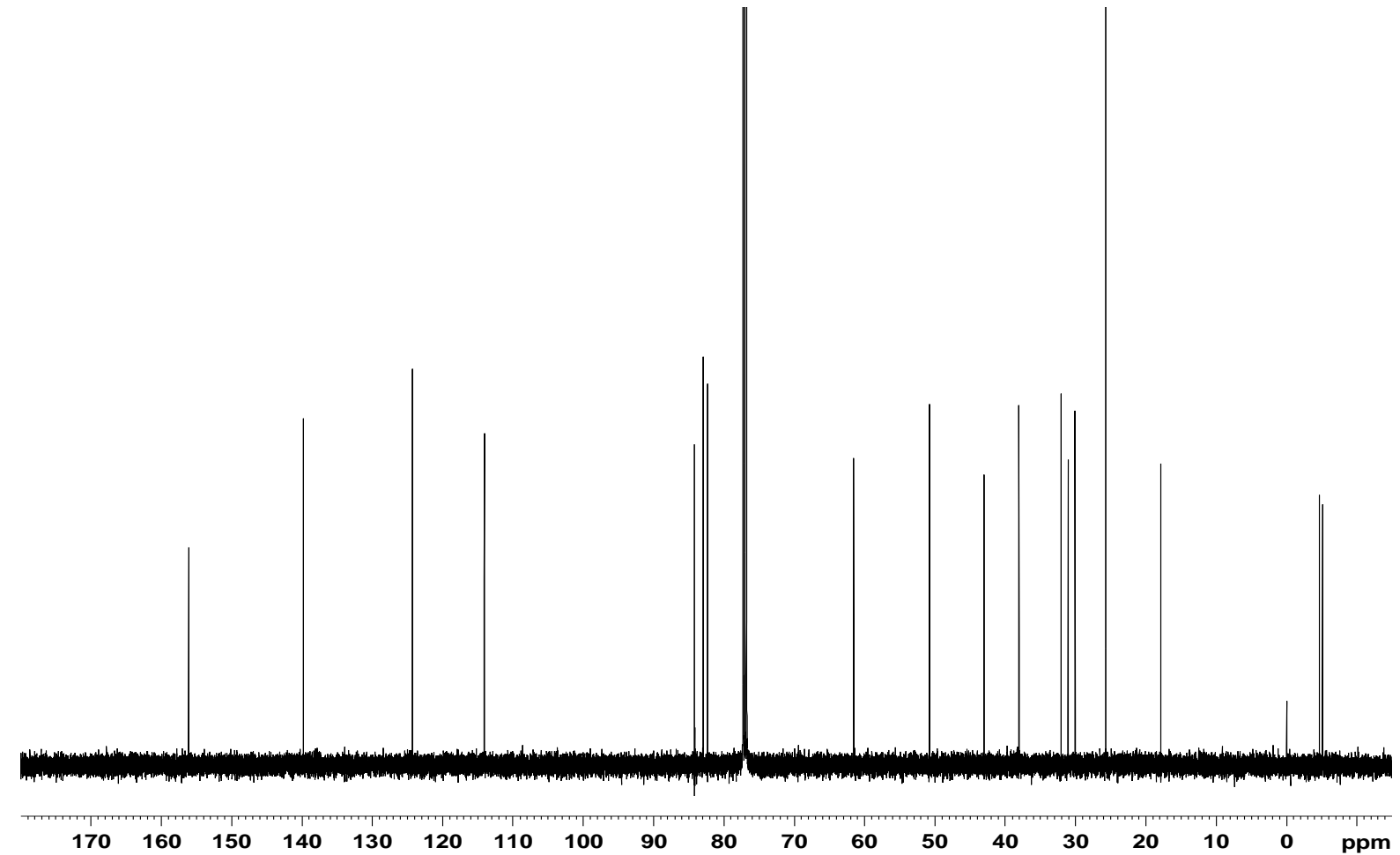


NMR spectra of $\left(1 R^{*}, 2 S^{*}, 3 a^{\prime} S^{*}, 5 S^{*}, 5 \mathrm{a}^{\prime} R^{*}\right)$-5-(tert-butyldimethylsilyl)oxy-2',3',3a',4'-tetrahydro2-vinyl-5' $H$-spiro[cyclopentane-1,1'-pentalene]-2,5'-diol (29)

${ }^{1} \mathrm{H} \mathrm{NMR}\left(\mathrm{CDCl}_{3}, 500 \mathrm{MHz}\right)$
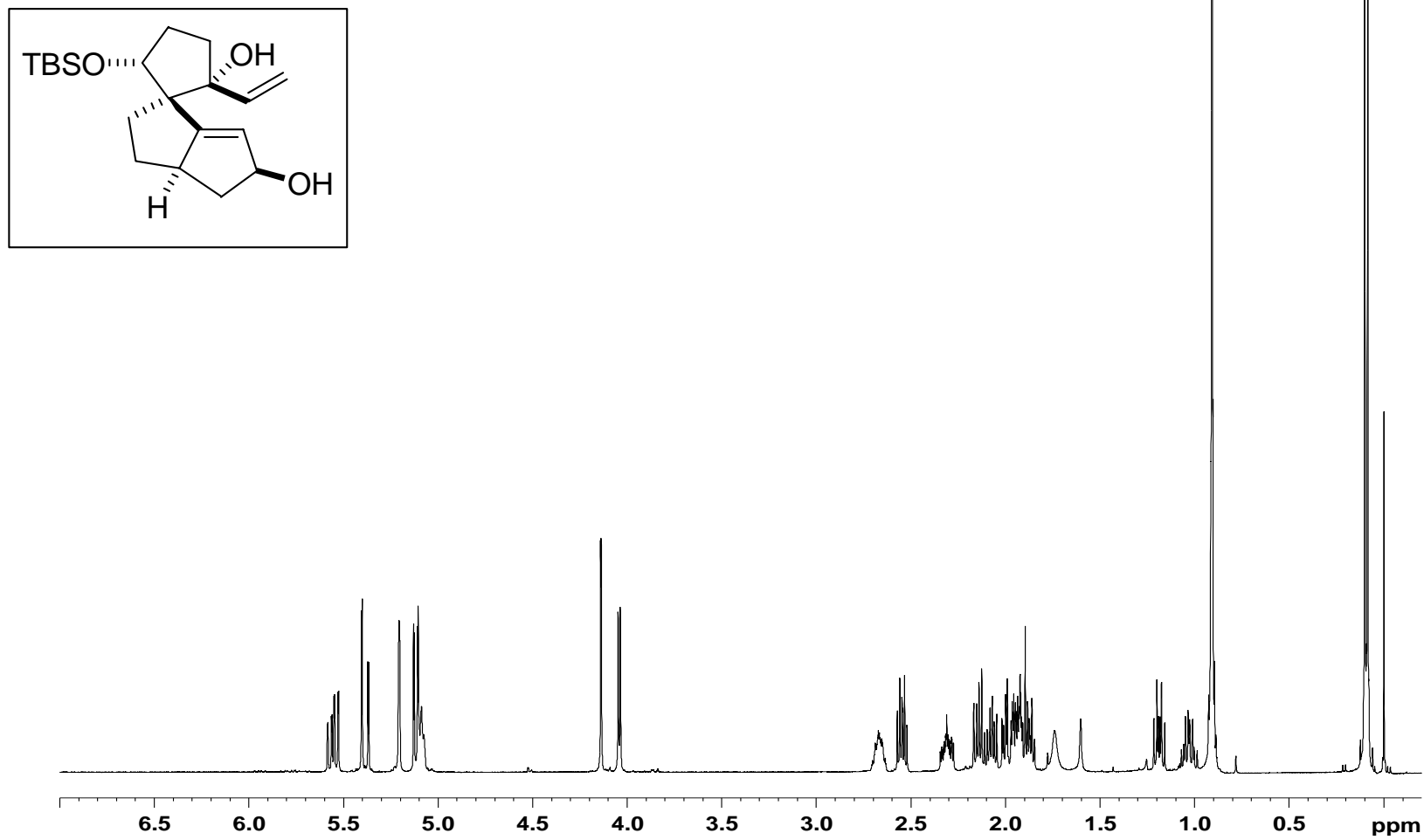

${ }^{13} \mathrm{C} \mathrm{NMR}\left(\mathrm{CDCl}_{3}, 125 \mathrm{MHz}\right)$

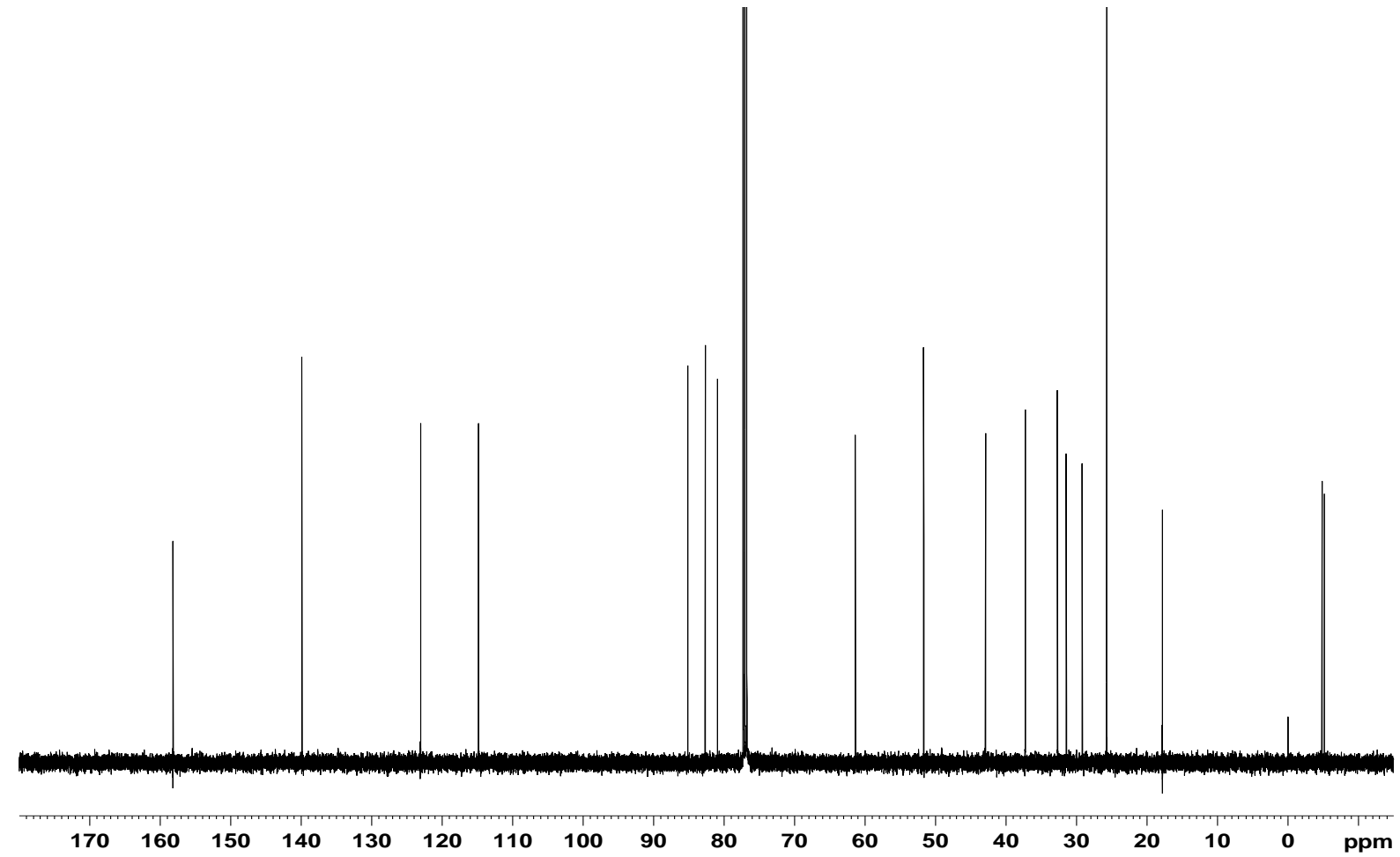


NMR spectra of $\left(1 R^{*}, 2 S^{*}, 3 a^{\prime} R^{*}, 5 S^{*}, 5 \mathrm{a}^{\prime} S^{*}\right)$-5-(tert-butyldimethylsilyl)oxy-2',3',3a',4'-tetrahydro5'-(methoxymethoxy)-2-vinyl-5' $H$-spiro[cyclopentane-1,1'-pentalen]-2-ol (30)

${ }^{1} \mathrm{H} \mathrm{NMR}\left(\mathrm{CDCl}_{3}, 500 \mathrm{MHz}\right)$
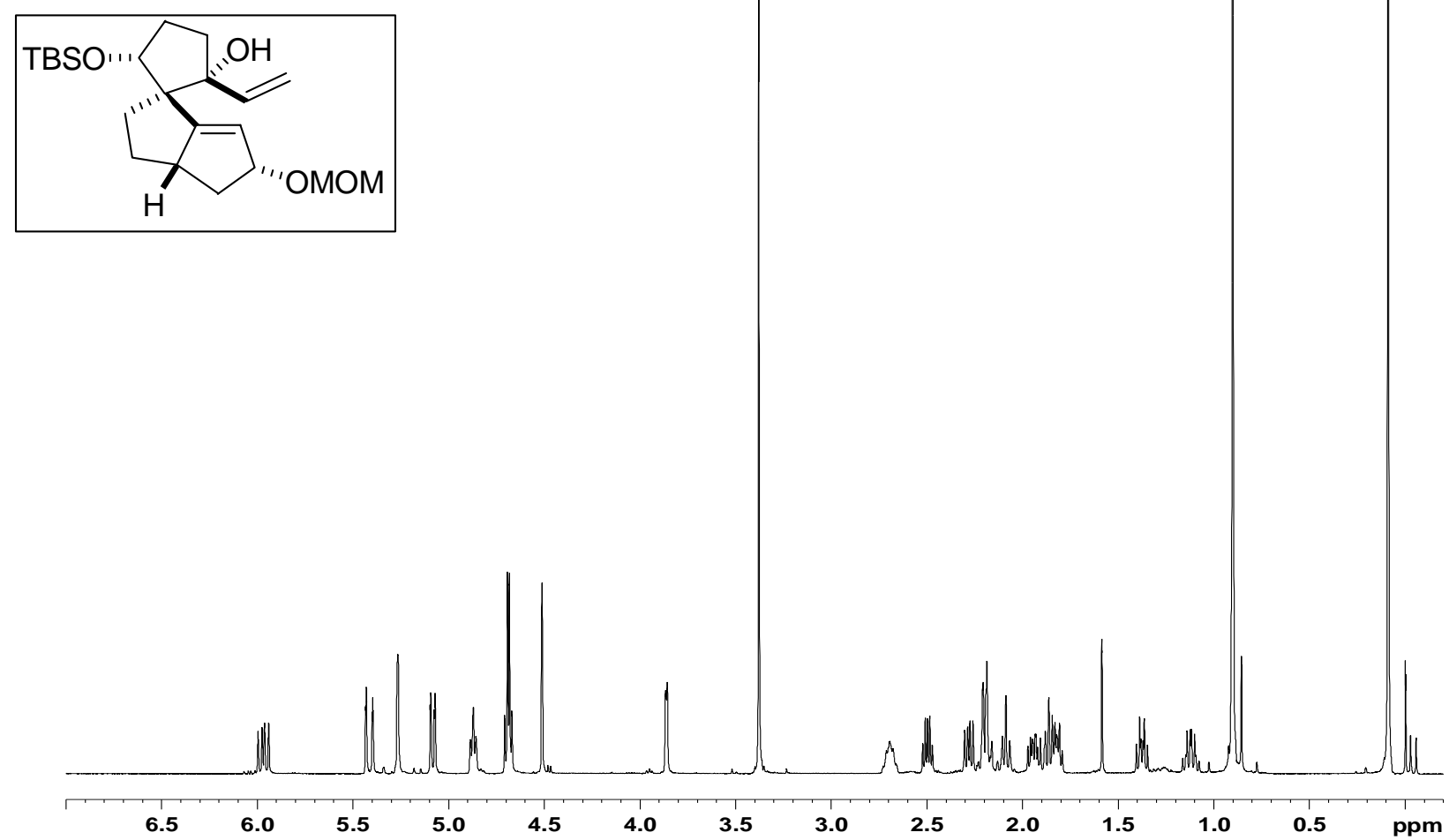

${ }^{13} \mathrm{C} \mathrm{NMR}\left(\mathrm{CDCl}_{3}, 125 \mathrm{MHz}\right)$

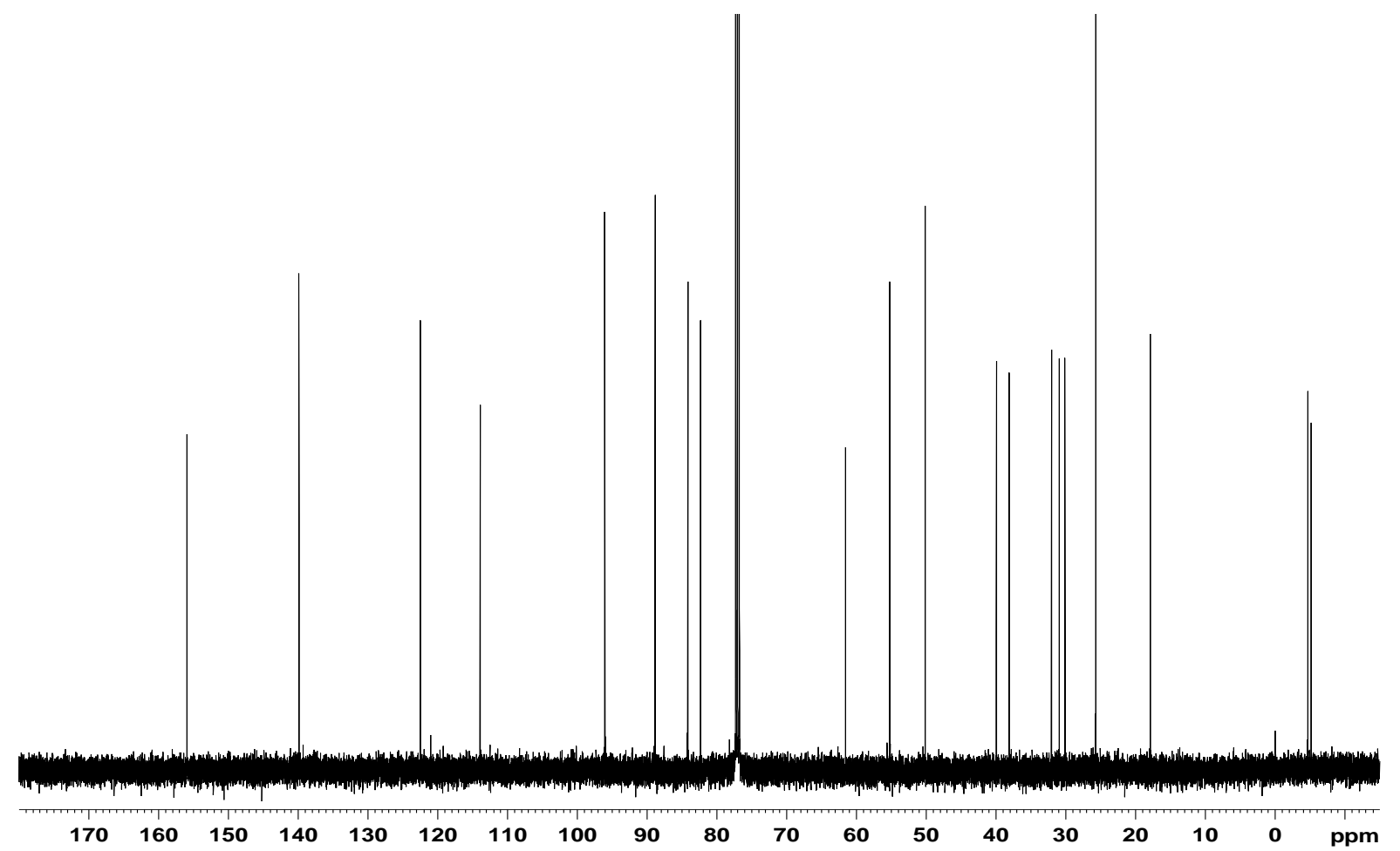


$\left(2 a R^{*}, 4 S^{*}, 4 a R^{*}, 10 S^{*}\right)-10$-(tert-butyldimethylsilyl)oxy-1,2,2a,3,4,4a,5,6,9,10-decahydro-4-(methoxymethoxy)cyclonona $[c d]$ pentalen-7(8H)-one (31)

${ }^{1} \mathrm{H} \mathrm{NMR}\left(\mathrm{CDCl}_{3}, 500 \mathrm{MHz}\right)$
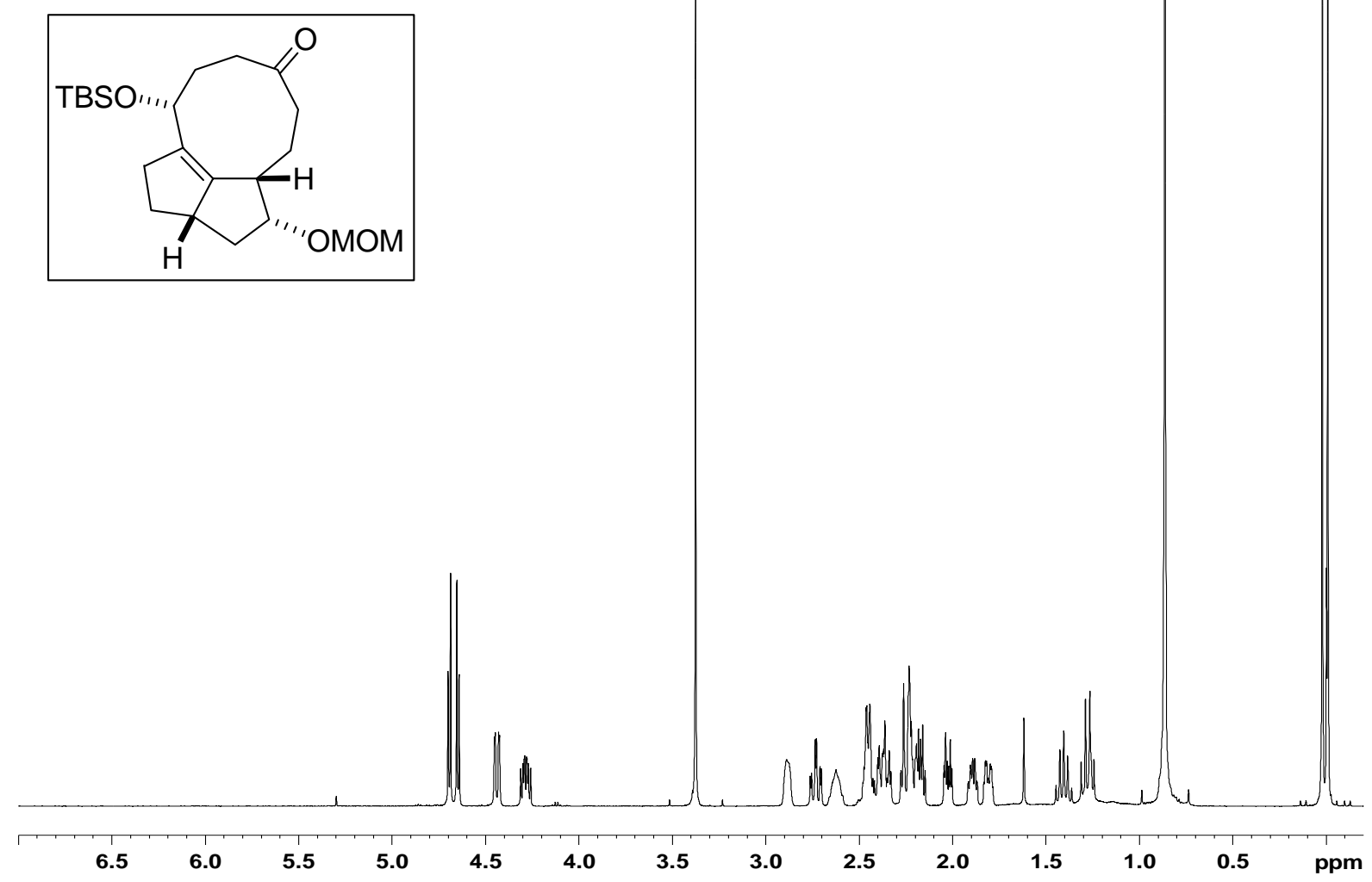

${ }^{13} \mathrm{C} \mathrm{NMR}\left(\mathrm{CDCl}_{3}, 125 \mathrm{MHz}\right)$

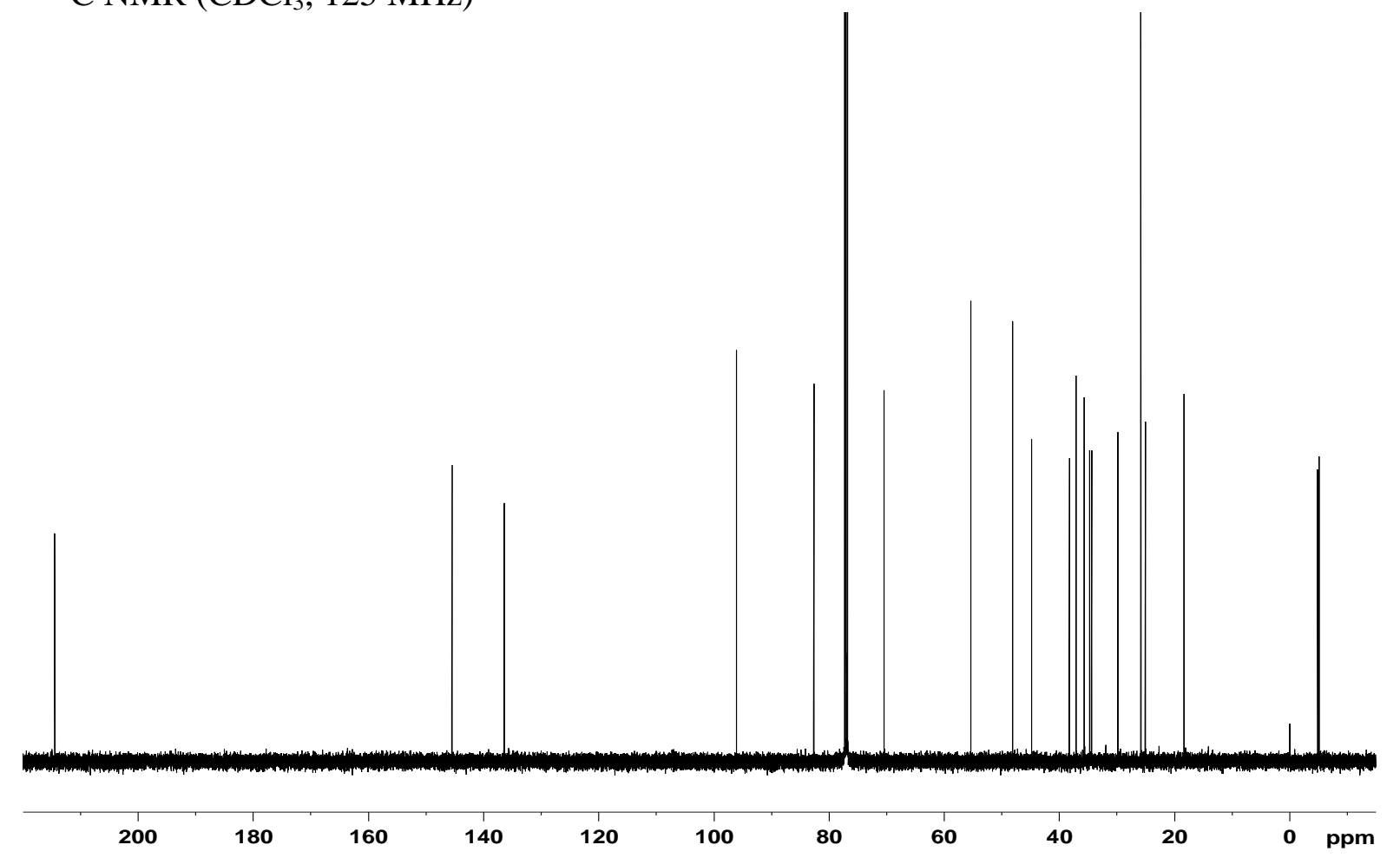


ORTEP plot of $\left(1 R^{*}, 2 R^{*}, 3 a^{\prime} R^{*}, 5 R^{*}\right)$-5-(tert-butyldimethylsilyl)oxy-2',3',3a',4'-tetrahydro-2hydroxy-2-vinyl-5' $H$-spiro[cyclopentane-1,1'-pentalen]-5'-one (15)

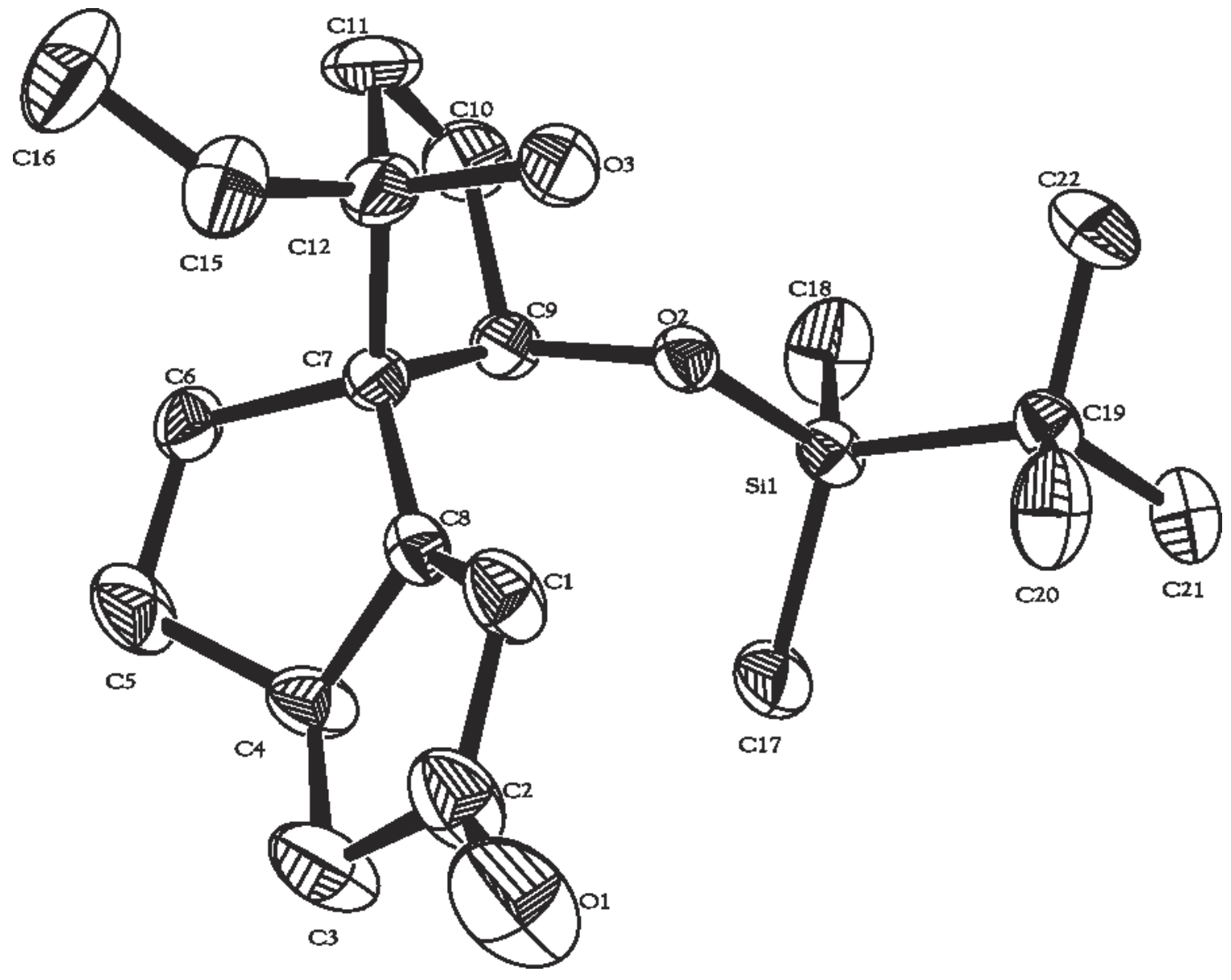


ORTEP plot of $\left(4 a R^{*}, 7 R^{*}, 7 a R^{*}, 9 a R^{*}\right)-7-(t e r t-b u t y l d i m e t h y l s i l y l) o x y-3,4,4 a, 5,6,7,9,9 a-o c t a h y d r o-$ 4a-hydroxy-1H-cyclopent $[k l]$-as-indacen-2(8H)-one (17)

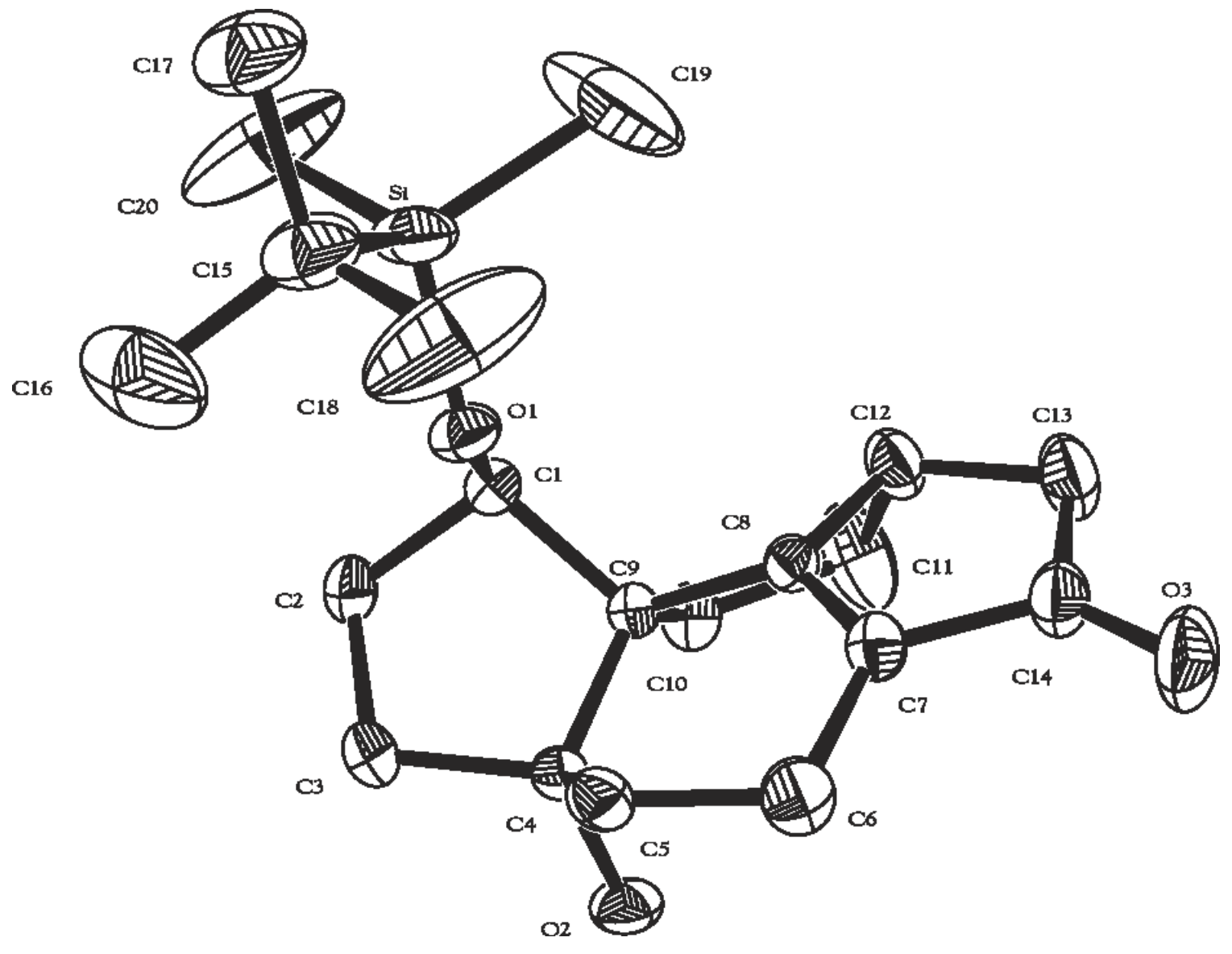


ORTEP plot of $\left(2 a R^{*}, 4 a S^{*}, 5 R^{*}, 6 a R^{*}, 9 a S^{*}, 9 b R^{*}\right)-5$-(tert-butyldimethylsilyl)oxy-2,2a,3,4,4a,5,6,6a, 9,9a-decahydro-1H-pentaleno[1,6- $d c]$ indene-1,7(8H)-dione (18)

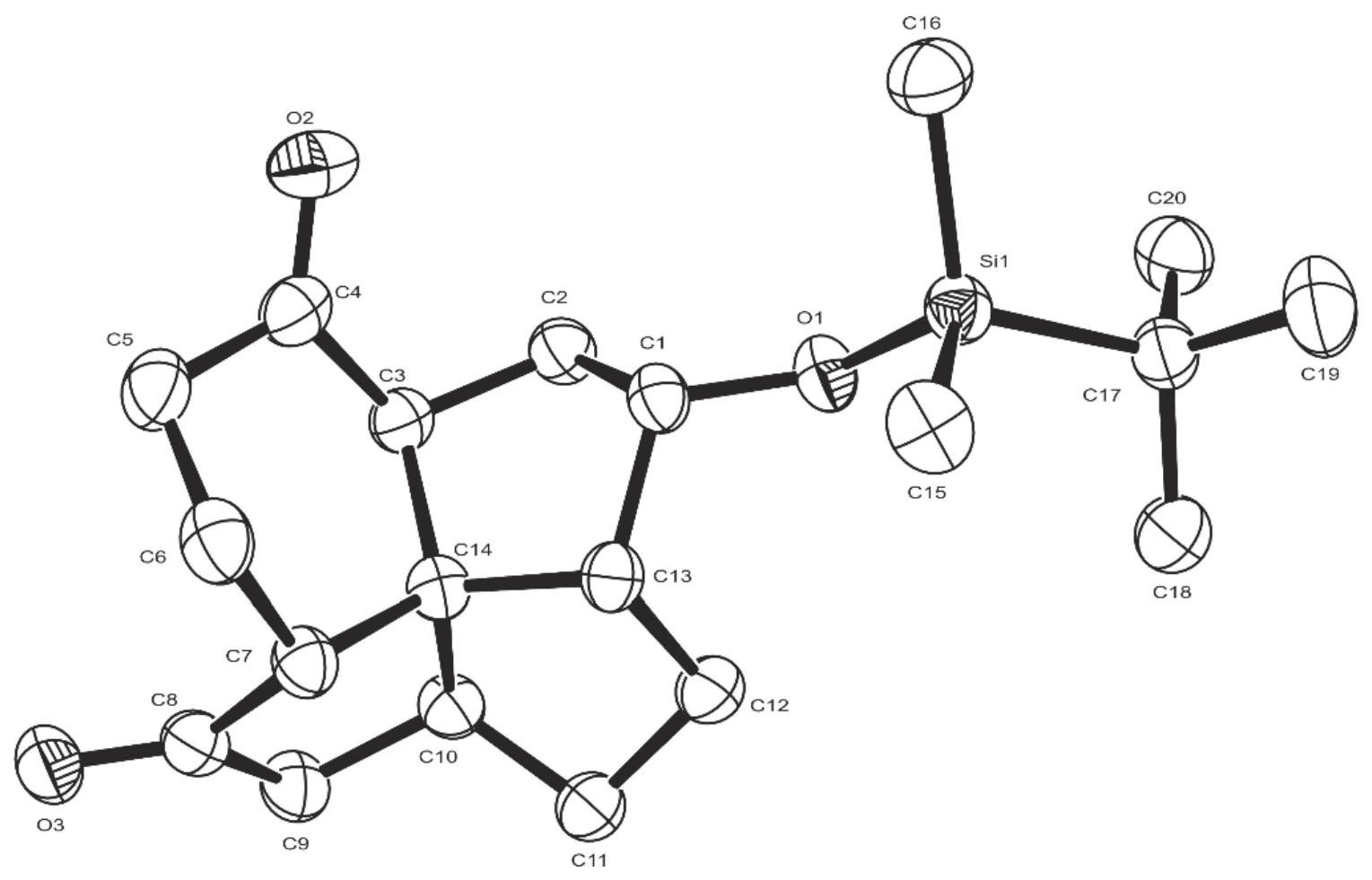

